\title{
Specialization in somatostatin subnetworks in sensory and association cortex
}

\author{
Christine F. Khoury ${ }^{1,2}$, Noelle G. Fala ${ }^{2}$, Caroline A. Runyan*1,2 \\ ${ }^{1}$ Center for the Neural Basis of Cognition, ${ }^{2}$ Department of Neuroscience, University of Pittsburgh \\ ${ }^{*}$ Corresponding Author: Caroline A. Runyan, runyan@pitt.edu
}

(1)

\section{Abstract}

Behavioral states profoundly affect cortical population activity dynamics and coding, yet the local circuits mediating these shifts are poorly understood, especially outside of sensory cortex. Somatostatin-expressing (SOM) inhibitory interneurons are highly sensitive to changes in brain state, and are well situated to modulate local population activity patterns. Here, we used twophoton calcium imaging to record the spike-related activity in populations of SOM and Non-SOM neurons in auditory cortex (AC) and posterior parietal cortex (PPC), to compare their functional interactions and activity modulation by behavioral state in sensory and association cortex. We have discovered both similarities and differences in the functional characteristics of SOM and Non-SOM populations across areas. In both regions, SOM neurons were more highly coordinated as populations than Non-SOM neurons. In PPC, SOM and Non-SOM neurons were positively modulated by increased arousal, while the effects of arousal in AC neurons were more diverse. Strikingly, the spatial structure of shared variability was different in AC and PPC: pairwise correlations in all cell types decayed rapidly with distance in AC but remained high over greater distances in PPC. Our results imply both generalization and specialization in the functional structure of excitatory and inhibitory subnetworks in sensory and association cortex.

\section{Introduction}

The mammalian neocortex is a hierarchically organized and highly interconnected network that can be subdivided into regions with functional specializations. Sensory cortices process incoming signals from single modalities, while downstream association areas integrate input from multiple modalities to generate behaviors. Incoming signals interact with rich, ongoing population activity dynamics throughout the brain, which are profoundly impacted by changes in behavioral and internal state.

The relationship between behavioral state and population activity dynamics has been most thoroughly explored in sensory cortex. In rodent sensory cortices, increased arousal has effects on cortical activity that are overall analogous to those of spatial attention in the primate visual system (1-5), including decorrelation of local population activity (6-9), enhanced response gain (10-14) and an improved signal:noise ratio $(6,13,15)$. However, even across sensory cortices, the effects of arousal on neural activity are not uniform (16). For example, in auditory cortex (AC), but not other modalities, the effects of arousal are complicated by negative motor-related feedback from motor cortex through local inhibitory interneurons $(8,17,18)$. Despite these differences, aroused and engaged behavioral states are associated with improved sensory 
decoding across sensory cortices of multiple modalities. The circuits underlying these arousalrelated effects are not fully understood, though inhibitory interneurons are likely to play important roles in controlling state-dependent shifts in population coding.

Inhibitory interneurons are critical components of local circuits, and well-situated to mediate many of the effects of internal state transitions on stimulus coding and population activity dynamics. The inhibitory cell class can be divided into three nonoverlapping subtypes, which express parvalbumin (PV), somatostatin (SOM), or vasoactive intestinal peptide (VIP), and participate in distinct local circuit motifs (19). Internal state transitions profoundly impact the activity of inhibitory interneurons. SOM neurons are particularly sensitive to internal state fluctuations, through direct activation of cholinergic and noradrenergic receptors (20-25), and through inhibition by VIP neurons $(11,26-30)$. In turn, SOM neurons can powerfully influence local networks, densely innervating the local excitatory population $(26,31,32)$, sharpening stimulus selectivity and response reliability (33-35), modulating local population activity dynamics $(22,36)$, and flexibly modulating excitatory responses to stimuli based on behavioral relevance $(37,38)$.

The local-circuit functions of SOM neurons outside of sensory cortex remain largely uncharacterized, though the relative density of SOM to PV neurons is known to increase in association relative to sensory cortices $(39,40)$. Theoretical work suggests that this difference contributes to flexible coding properties of association cortex (38). Though many aspects of inhibitory microcircuitry are conserved across all of cortex, patterns of their synaptic connectivity and functional modulation by brain state may be tailored to specific regions' primary roles, such

60 as in sensory processing or in performing flexible sensorimotor transformations.

Here, we tested the hypotheses that local inhibitory population activity in sensory and association cortex is differentially structured across neurons and differently modulated by arousal. We transgenically labeled SOM neurons in auditory and posterior parietal cortices and performed two-photon calcium imaging in both areas to relate SOM and Non-SOM activity to behavioral correlates of arousal, and to local population activity. We reveal clear area-specific differences in the modulation of SOM and Non-SOM neurons with changes in arousal. Furthermore, while the spatial scale of SOM and Non-SOM functional subnetworks differed in sensory and association cortex, commonalities in the activity relationships between SOM and other neurons were also evident across areas. Together, our results suggest that both specializations and generalized principles in the function of SOM neurons apply across cortex.

\section{Results}

To determine whether the effects of arousal on cell-type specific activity are conserved across sensory and association cortex, we compared spontaneous and sensory-evoked activity in auditory cortex (AC) and posterior parietal cortex (PPC) in six mice of both sexes. We used twophoton calcium imaging in superficial cortex to measure the spike-related activity of neurons positive and negative for the red fluorophore tdTomato, which was expressed transgenically in somatostatin-positive (SOM) neurons $(41,42)$. We virally expressed the genetically encoded calcium indicator GCaMP6f in all layer 2/3 neurons of AC and PPC in each mouse (43). Mice were head-fixed and ran voluntarily on a spherical treadmill; running velocity and pupil area were tracked throughout imaging sessions (Figure 1A-B). 

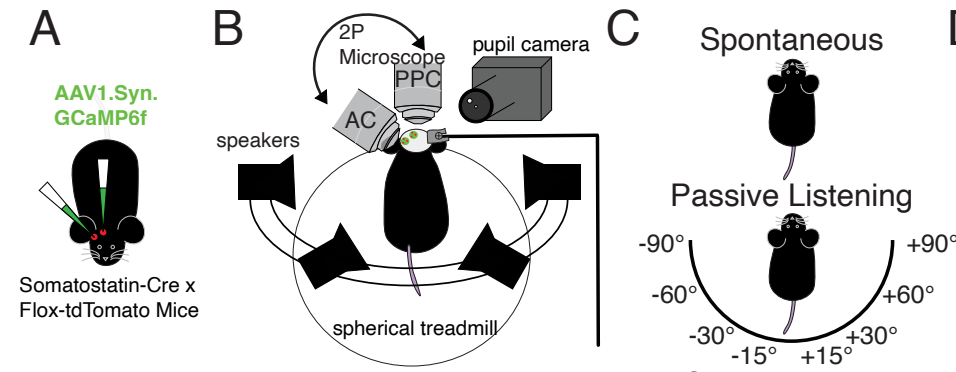

Sound locations
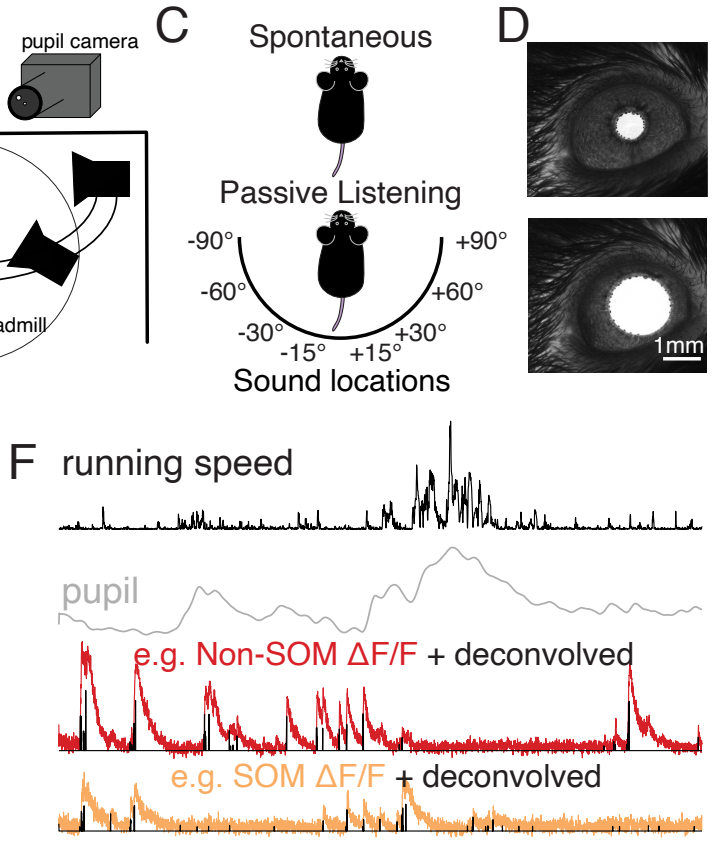

$100 \mathrm{~cm} / \mathrm{s} \mid 200 \% \Delta \mathrm{F} / \mathrm{F}$

$50 \% \max 2$ a.u.

$30 \mathrm{~s}$

\section{Posterior Parietal}
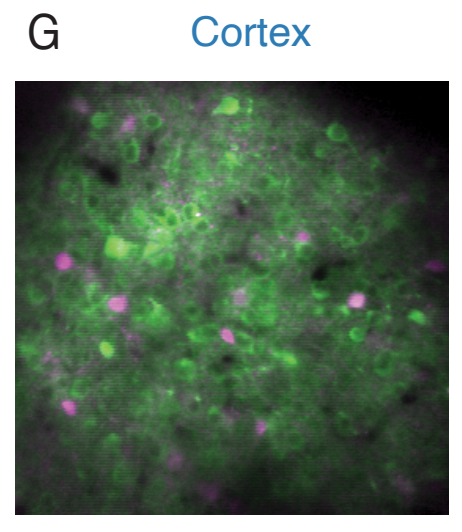

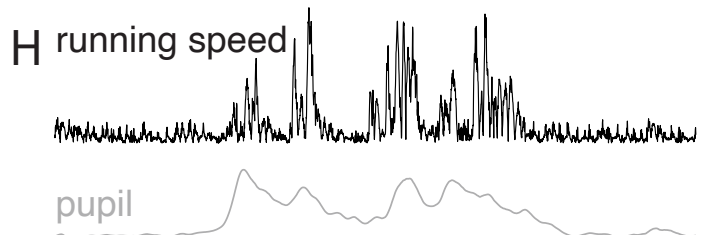

e.g. Non-SOM $\triangle F / F+$ deconvolved

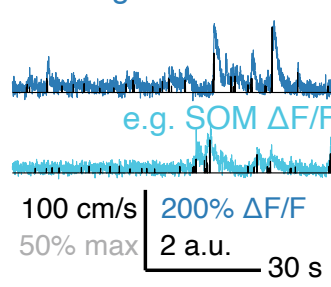

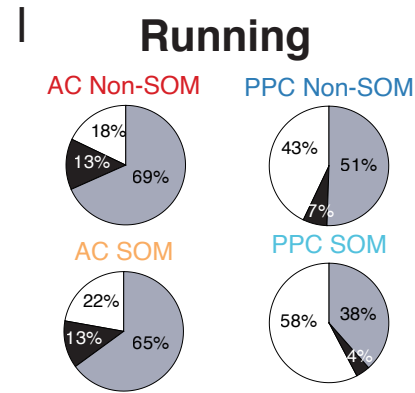

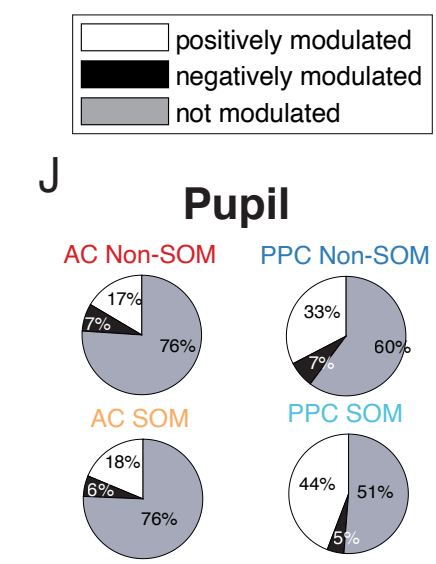

K
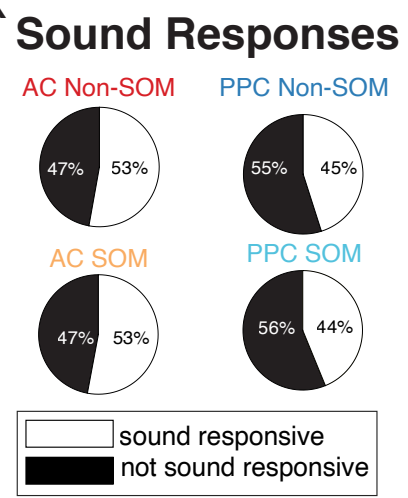

Figure 1: Imaging spike-related activity in SOM and Non-SOM neurons during behavioral state transitions. (A) Viral injections and cranial windows were made over auditory cortex $(A C)$ and posterior parietal cortex (PPC) in each SOM-tdTomato mouse. (B) In imaging sessions, mice were headfixed over a spherical treadmill and allowed to run voluntarily. Four speakers arranged around the head presented sound stimuli. An infrared camera was used to image the pupil, and a rotating two-photon microscope was focused on either AC or PPC on a given imaging day. (C) Each imaging session included "Spontaneous" and "Passive Listening" contexts, without or with randomly presented sound stimuli from each of eight locations, respectively. (D) Pupil area was monitored via the pupil camera, scalebar in bottom image applies to top (constricted pupil) and bottom (dilated pupil). (E) Example field of view from auditory cortex, with intermingled tdTomato+/SOM+ (magenta) and tdTomato-/SOM- neurons, co-expressing GCaMP6f (green). (F) Example aligned behavioral and neural signals collected during the imaging session in $\mathrm{E}$, including running speed $(\mathrm{cm} / \mathrm{s})$, normalized pupil area, dF/F from a Non-SOM (red) and SOM neuron (orange), each overlaid with the neuron's deconvolved estimated spike rates. $(G)$ As in $E$, for an example posterior parietal cortex field of view. (H) As in F, for the PPC field of view in G. (I) Proportions of Non-SOM and SOM neurons in AC (left) and PPC (right) with significant positive (white), negative (black), or no modulation by mouse's running onset. AC Non-SOM N=2645; AC SOM N=359; PPC Non-SOM N=4719; PPC SOM N=525. (J) As in I, for pupil dilation. (K) Proportion of Non-SOM and SOM neurons in AC and PPC that were significantly sound responsive to at least one location (white) or not significantly sound responsive (black). 
During each imaging session, we imaged AC or PPC during the "spontaneous" context, where the mouse ran freely on the spherical treadmill in the absence of sensory stimulation, and during "passive listening", where sounds were presented from eight separate locations (Figure 1B-C). We first examined how changes in pupil area and running speed corresponded with changes in activity of individual neurons during the spontaneous context. Throughout imaging sessions, mice spontaneously transitioned between behavioral states: stillness and running, and pupil constriction and dilation (Figure 1F, H, Figure S1A). To quantify the effect of behavioral state transitions on individual neurons' activity, we identified timepoints during the spontaneous context when either running speed or pupil area increased (Materials and Methods 5.2-5.3). The frequency of running bouts and dilation events was similar during PPC and AC imaging sessions $(p=.59$ and $p=.65$ respectively, Table S1). Mice initiated running bouts at a rate of $.915 \pm .095$

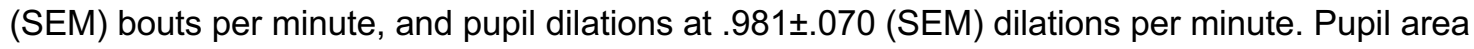
and running speed were higher during the passive listening context relative to the spontaneous behavior context (Figure S1).

To determine how transitions in behavioral state affected neural activity, we first quantified the effects of transitions from stationary to running on individual cells. We compared mean activity of each neuron (deconvolved estimated spike rates, here and throughout) in the 3-s time window following running bout onset to the 1-s time window prior to running bout onset (Figure 11, See Materials and Methods 5.2). Pupil modulation was calculated similarly, based on transitions in pupil area from constricted to dilated (Figure 1D, J; Materials and Methods, 5.3). We compared each neuron's running and pupil modulation to shuffled distributions, where activity and behavioral data were time shifted by random intervals, and classified each neuron as positively, negatively, or not modulated by behavioral state transition. Larger proportions of the SOM and Non-SOM populations were modulated by both pupil dilations and running bout onsets in PPC than in AC (Figure 1I-J), suggesting that the effects of arousal on spontaneous neural activity are not uniform across areas.

\section{Sound modulation of SOM and Non-SOM neurons' activity}

To compare the auditory spatial selectivity of SOM and Non-SOM neurons in AC and PPC, sound stimuli (dynamic moving ripples) presented from eight distinct locations, centered on the mouse's head (Figure 1B-C, See Materials and Methods 3.4). We focused on spatial modulation of auditory stimuli due to PPC's role in spatial auditory processing during active behaviors (44). Sound responsiveness was determined similarly to pupil area and running speed modulation. We computed the mean difference in deconvolved activity during each sound presentation and the pre-stimulus period for each sound stimulus location, and compared to a shuffled distribution for each neuron (Materials and Methods 5.1). Large proportions of neurons in both areas were significantly sound responsive, but a greater proportion of AC SOM and Non-SOM neurons were sound responsive compared to PPC ( $53 \%$ of AC SOM and 53\% of AC Non-SOM relative to $45 \%$ of PPC SOM and $44 \%$ of PPC Non-SOM neurons, Figure $1 \mathrm{~K}$ ), as expected.

124 Differing effects of arousal state on spontaneous activity of SOM and Non-SOM neurons in AC 125 and $P P C$

126 To further characterize the relationship between arousal state transitions and spontaneous 127 activity of single neurons in AC and PPC in the spontaneous context, we continued to focus on 
"running bouts", when the mouse's running speed rapidly increased, to define behavioral state transitions (Materials and Methods 5.2). We aligned and averaged the pupil area to running bout onsets and observed that pupil area also increased during running bouts, though with a slower time course (Figure 2A). We also aligned and averaged the activity of AC SOM, AC Non-SOM, PPC SOM, and PPC Non-SOM populations (AC SOM and Non-SOM, n=24; PPC SOM and NonSOM, $n=20$ imaging sessions from 6 mice) to running bout onset (Figure $2 A)$. While there was little net change in the overall activity of AC populations relative to PPC, examining the activity of each individual neuron revealed populations of SOM and Non-SOM neurons that increased or decreased activity upon running onset. In PPC on the other hand, most Non-SOM and SOM neurons increased activity at running onset (Figure 2B). We observed analogous results when activity was aligned to transitions in pupil area (Figure S2A-B). While transitions in pupil area corresponded with increases in running speed (Figure 2A, Figure S2A, D-E), the two signals varied on different timescales. Running speed was characterized by a narrower autocorrelation function than pupil area, demonstrating that running speed varied on a faster timescale than pupil area (Figure S2C). To directly relate the two signals to each other, we computed the crosscorrelation between running speed and pupil area at different time lags. While the two signals were highly correlated overall, the peak cross-correlation occurred at a 1.27s time lag, with running preceding pupil area, further demonstrating the slower timescale of pupil changes relative to running speed changes (Figure S2D).

Because of the slow time course of pupil area changes, we also characterized single neuron activity during periods of sustained pupil constriction and dilation. We classified pupil measurements as low, transitional, or high arousal states (Figure 2C, Materials and Methods 6.1), and focused analyses on the low and high arousal states. As expected from the positive correlation between running speed and pupil area, running speed was higher during the pupildefined high arousal than low arousal states ( $p<.001$, Figure S2E). No running speed differences were observed between AC and PPC imaging sessions in the high $(p=.087)$ or low arousal periods $(p=.89$ ). Mice ran on average $48.24 \pm 4.77 \mathrm{~cm} / \mathrm{s}$ (SEM) during the high arousal state, and $7.71 \pm 1.29 \mathrm{~cm} / \mathrm{s}$ (SEM) during the low arousal state (Figure S2E) and spent similar amounts of time in the low and high arousal states during AC and PPC imaging sessions (Table S1).

Firing rates were elevated overall in high arousal states for SOM and Non-SOM neurons in both regions $(p<.001$; Figure $2 \mathrm{D}-\mathrm{F}$, Table $\mathrm{S} 2)$. To compare arousal modulation of activity in each population of neurons, we next computed the Arousal Modulation Index (Materials and Methods 6.2 ), which could vary from -1 to +1 , with -1 indicating greater mean activity in the low arousal period, and +1 indicating greater activity in the high arousal period. The Arousal Modulation Index differed significantly across the four groups (Figure 2G, $p<0.001$, Kruskal Wallis, Table S2). Arousal modulation of PPC SOM and Non-SOM neurons was significantly greater than arousal modulation of AC SOM and Non-SOM neurons ( $p<.001)$, but did not differ between AC SOM and Non-SOM neurons $(p=.22)$. In PPC, SOM neurons were significantly more modulated than NonSOM neurons $(p<.001)$.

Finally, to consider the full time-varying relationship between ongoing neural activity and behavioral correlates of arousal (running speed and pupil area), we correlated each neuron's activity and pupil area or running speed, across the entire spontaneous behavioral context (Figure $2 \mathrm{H}-\mathrm{I}$ ). Pearson correlations between activity and pupil area/running speed were significantly different across groups (pupil area and running speed, $p<0.001$, Kruskal-Wallis). Consistent with the above analyses based on state transitions (Figures $1 \mathrm{I}-\mathrm{J}, 2 \mathrm{~A}-\mathrm{F}$ ), the 

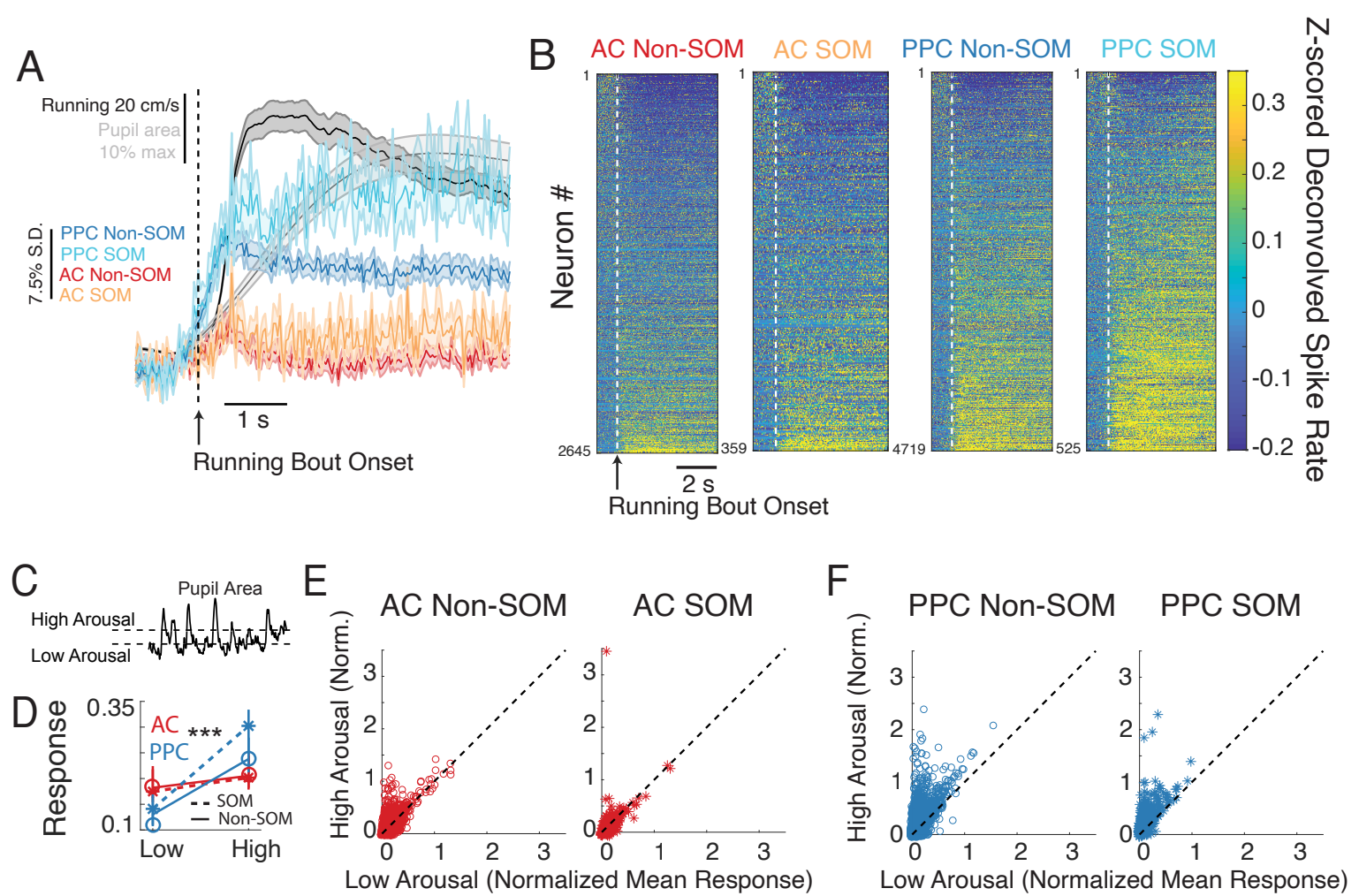

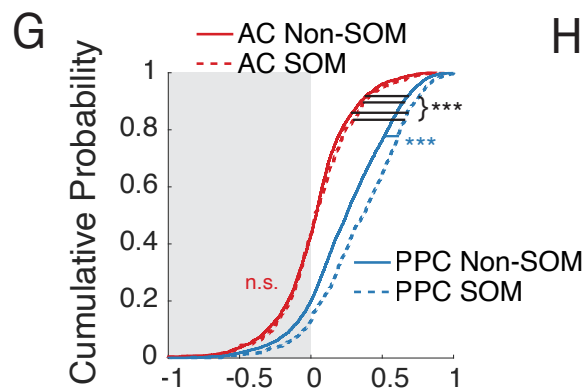

Arousal Modulation Index

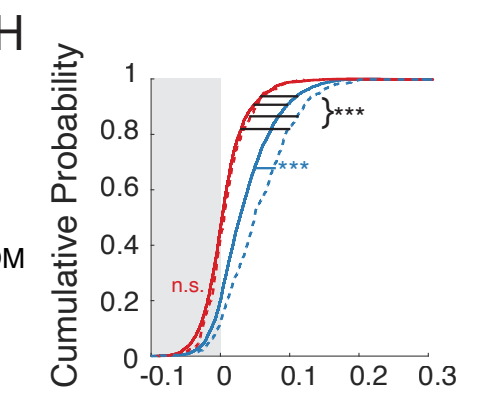

Activity-Pupil Correlation

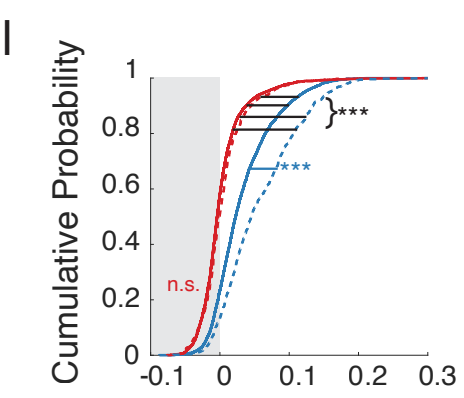

Activity-Running Correlation

Figure 2: Single cell activity modulation with increases in arousal. (A) Average z-scored deconvolved activity, running speed, and pupil area aligned on running bout onset. Red: AC Non-SOM neurons' activity ( $\mathrm{N}=24$ datasets), Orange: AC SOM neurons' activity ( $\mathrm{N}=24$ datasets), Dark Blue: PPC Non-SOM neurons' activity ( $\mathrm{N}=20$ datasets), Light Blue: PPC SOM neurons' activity ( $\mathrm{N}=20$ datasets), Black: Running speed ( $\mathrm{N}=44$ datasets), Gray: Pupil area ( $\mathrm{N}=44$ datasets). (B) Average $z$-scored deconvolved activity of individual neurons, from $L$ to R: all AC Non-SOM, AC SOM, PPC Non-SOM, and PPC SOM neurons aligned on running bout onset (AC Non-SOM N=2645; AC SOM N=359; PPC Non-SOM N=4719; PPC SOM N=525, in B-I). Neurons were sorted by running modulation. (C) Illustration of clustering that sorted pupil area during each imaging frame into low, transition, and high arousal states. (D) Mean responses of AC Non-SOM (red solid), AC SOM (red dotted), PPC Non-SOM (blue solid), and PPC SOM (blue dotted) neurons in low and high arousal states, as defined in C. (E) Left: Mean activity of AC Non-SOM neurons in high arousal state (classified with clustering as in C), plotted against mean activity in the low arousal state. Right: Mean activity of AC SOM neurons in high arousal vs low arousal states. (F) As in E, for PPC Non-SOM and SOM neurons. (G) Cumulative probability distribution of the arousal modulation index in AC Non-SOM (solid red), AC SOM (dotted red), PPC Non-SOM (solid blue), and PPC SOM (dotted blue) neurons. Arousal modulation index was calculated from the values in E-F, (High-Low)/(High+Low) for each neuron. $(\mathrm{H})$ Cumulative probability distribution of the Pearson correlation between each neuron's activity and pupil area, colors as in G. (I) Cumulative probability distribution of the Pearson correlation between each neuron's activity and running speed. Significance as indicated, permutation test, ${ }^{* \star *} p<.001$. Error bars: $95 \%$ bootstrapped confidence interval around the mean. See full summary of values and statistics in Tables S2-3. 
correlations between activity and running/pupil size were significantly greater in PPC SOM and Non-SOM neurons than AC SOM and Non-SOM neurons $(p<.001)$. In AC, SOM and Non-SOM neurons were similarly correlated to running/pupil size (pupil, $p=.063$, running, $p=.146$ ), while in PPC, SOM neurons were significantly more correlated with both running and pupil than were Non-SOM neurons $(p<.001$, Figure $2 \mathrm{H}-\mathrm{I}$, Table S3).

Taken together, our results indicate that SOM and Non-SOM neurons have heterogeneous activity relationships with arousal state in $A C$, whether defined by running speed or pupil area. In PPC, on the other hand, neural activity was strongly and positively related to increased arousal. In AC, no differences in arousal-modulation of SOM and Non-SOM neurons were evident, while in PPC, SOM neurons were more sensitive to increases in arousal than Non-SOM neurons. All analyses reported in Figure 2 used data collected during the spontaneous context to isolate the effects of arousal on spontaneous activity in the absence of sensory evoked responses. However, we obtained analogous results using data collected during the passive listening context, suggesting that the overall effects of arousal are similar with and without sensory stimulation (Figure S3, Tables S4-5).

\section{An encoding model to disentangle running speed and pupil size contributions to single cell activity}

Running speed and pupil area are strongly correlated signals but do vary on different timescales (Figure S2C-D) and have separable impacts on neural activity (6). Our analyses of ongoing spontaneous activity in AC and PPC hint at the possibility of separable effects of pupil area and running speed, as AC Non-SOM neurons' activity was more highly correlated with pupil area $(0.0057 \pm .00063$ SEM) than with running $(.0020 \pm .00066$ SEM, $p<.001)$.

To disentangle the relationships between neural activity, running speed, and pupil area, we used a generalized linear modeling (GLM) approach (45-47). We constructed an encoding model (GLM), that used sound stimulus timing and location, pupil area, and running velocity to predict the responses of individual SOM and Non-SOM neurons in AC and PPC, in the passive listening context (Figures 3A, S4). It should be noted that in the above analyses (Figure 1I, 2A-B,2I), we considered only the speed at which the mouse was running in any direction, as increased running speed is correlated with heightened arousal $(6,8,11,12,14,16)$. Because PPC neurons' activity can be selective for running direction $(45,48-51)$, in the GLM we used running velocity rather than speed to obtain more accurate predictions for each neuron (Materials and Methods 8.0, Figure S4).

To determine the relative contributions of pupil area and running velocity to neurons' activity, we compared the prediction performance of variants of the model that were missing either predictors based on pupil area or running velocity to the prediction performance of a model that included pupil area, running velocity, and sound stimulus predictors (Figure 3). For each neuron, we calculated running and pupil area contributions to its activity as the difference between the performance of the full model and the no-running and no-pupil models respectively (Materials and Methods 8.6). This approach allowed us to isolate the contributions of running velocity and pupil size that were not redundant with each other. For example, when pupil area is well-explained by running velocity, running predictors would account for the missing pupil area predictors, and the performance of the model would not be degraded compared to the full model that includes both pupil area and running velocity predictors. The model comparison revealed single neuron activity that could be explained uniquely by running and by pupil in both AC and PPC (Figure 3B-G, note 
A

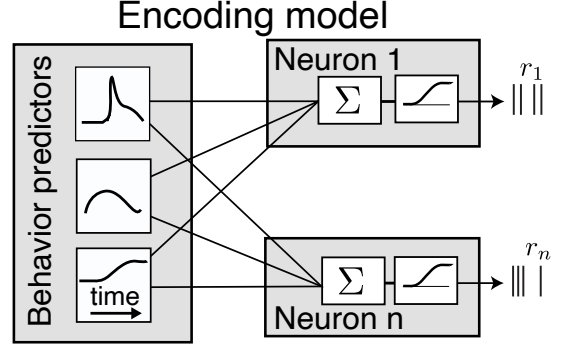

$\mathrm{B}$

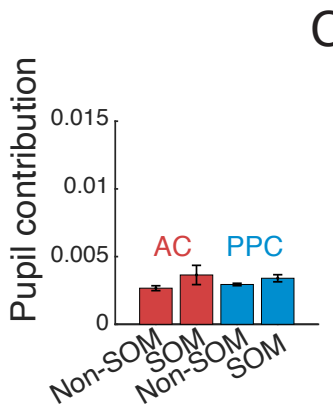

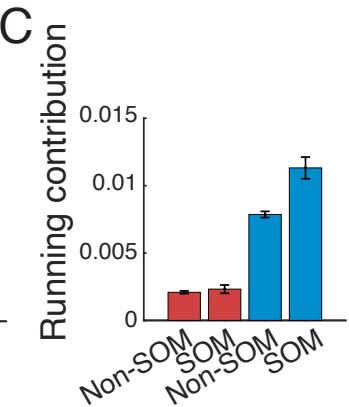

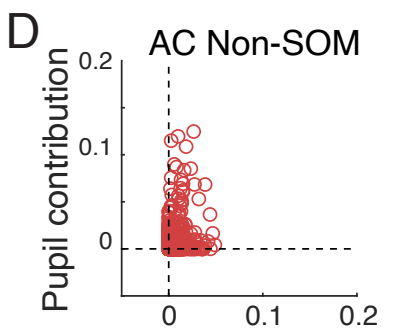

Running contribution

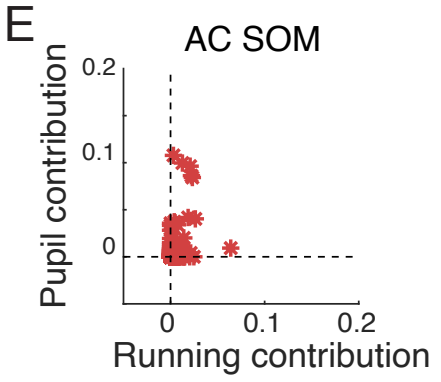

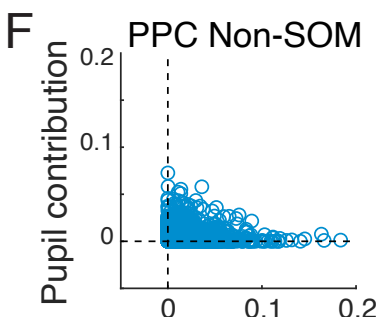

Running contribution

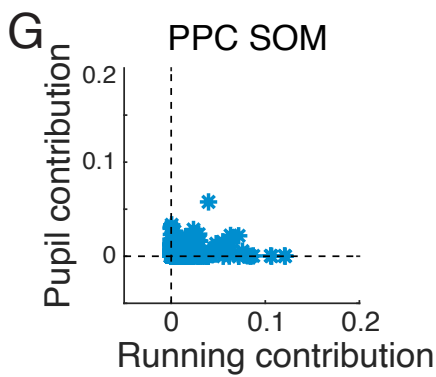

Figure 3: Using an Encoding Model to Disentange Running and Pupil Contributions to Single Cell Activity. (A) Schematic of Generalized Linear Model (GLM) used to determine contributions of pupil area and running velocity to neurons' activity. (B) Average contribution of pupil area to activity across all AC Non-SOM ( $\mathrm{N}=2645)$ and SOM ( $\mathrm{N}=359)$ neurons (red bars) and PPC Non-SOM $(\mathrm{N}=4525)$ and SOM $(\mathrm{N}=505)$ neurons (blue bars). Contribution was quantified as the improvement in model prediction performance of each neuron's activity when adding pupil area as a set of predictors. Errorbars indicate SEM (C) As in B, calculated when comparing model performance with and without running velocity predictors. (D) Pupil contribution plotted against running contribution for each AC Non-SOM neuron, calculated as in B. (E) Pupil contribution plotted against running contribution for each AC SOM neuron. (F-G) Pupil contribution plotted against running contribution for each PPC Non-SOM or SOM neuron. Sample sizes in B apply to all panels, full statistics in Table S6. 
216 neurons along the pupil and running contribution axes in $D-G)$. Contributions of pupil size to activity were slightly stronger in PPC than AC overall ( $p<0.05$, Table S6). Running contributions were stronger overall in PPC neurons than AC neurons $(p<0.001)$, and even higher among PPC SOM neurons than PPC Non-SOM neurons ( $p<0.001$ Figure $3 C, F, G$, Table $S 6$ ). The elevated running velocity contribution in PPC neurons is consistent with PPC's role in sensorimotor transformations and navigation $(45,52,53)$.

Characterizing sound location responses from AC and PPC in low and high arousal

To characterize sound location specificity of responses in AC and PPC, we computed a location selectivity index (LSI, Figure S5A-D, See; Materials and Methods 10.0). PPC neurons overall were less selective for location than AC neurons ( $p<0.01$, Table S7), and PPC sound responses were less reliable ( $p<0.001$, Table S8). Within both regions, Non-SOM neurons were more selective for sound location than SOM neurons ( $p<0.001$, Figure S5B-C, Table S7). Based on the differences in location selectivity and response reliability, we expected population activity to represent sound location more accurately in AC than PPC.

We built decoders based on population activity that predicted the most likely sound stimulus location using the activity of subsets of neurons ("best cells", SOM neurons, or Non-SOM neurons). The number of neurons was matched for sample size across cell types for valid comparisons of population decoding performance. Each decoder was based on a Bayesian inversion of our encoding model (Figure 4A, Materials and Methods, 9.0). The posterior probability of each stimulus $(\mathrm{p}(s \mid r)$, where $s$ is the stimulus and $r$ is the response probability of the simultaneously imaged neurons) was then computed cumulatively at each timepoint using all previous timepoints in the trial. Decoder performance was quantified as the fraction of trials where the stimulus with the maximal posterior probability matched the actual presented stimulus.

First, to compare the overall ability of AC and PPC to represent sound location, we used activity of only the "best" cells (i.e., individual neurons with the best sound location decoding themselves) regardless of cell type in the population decoder. The best cell decoder's performance was above chance when using activity from both AC and PPC (Figure 4C-D, Materials and Methods, 9.0). However, as expected, AC decoding was more accurate than PPC decoding ( $p<0.001$; Figure 4C-D, Materials and Methods 9.0, Table S9). Next, to compare sound location decoding accuracy in the SOM and Non-SOM populations, we built population decoders based on n-matched populations of SOM and Non-SOM neurons from each dataset. In both areas, sound location decoding was similar in SOM and Non-SOM populations (Figure 4C), and both cell type populations from AC outperformed decoding based on PPC populations ( $p<0.001$, Figure 4C-D). All cell-type specific, subsampled population decoders performed significantly worse than the decoders based on an n-matched population of "best" neurons $(p<0.001$; Figure 4D, See Materials and Methods 9.0). The same was true when instead of decoding left or right, we decoded specific contralateral sound locations (Figure S6). To summarize, sound location was better represented in AC than PPC, and SOM and n-matched Non-SOM populations in both areas were similarly informative about sound location. A sparse code for sound location was evident in both AC and PPC, as small numbers of highly tuned "best" neurons more accurately encoded sound location than random subsamples of the population.

257 To determine how arousal-related activity modulation affects sensory representations in AC and 258 PPC, we also trained and tested decoders in low and high arousal periods. Sound stimulus trials 


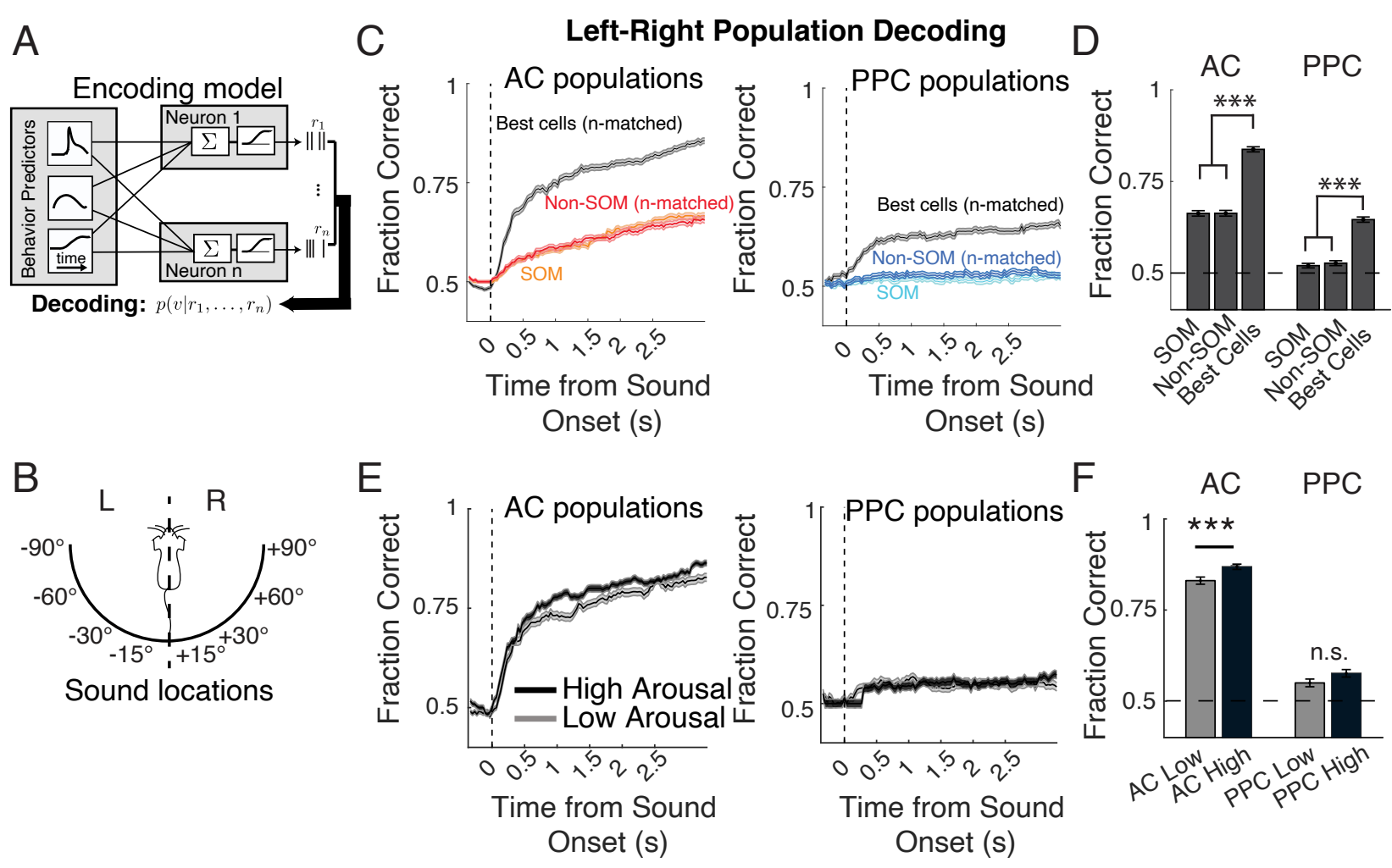

Figure 4: Decoding Sound Location from AC and PPC Population Activity. (A) The encoding model was trained on all trials that included all arousal levels, and inverted using Bayes Rule to compute the posterior probability of auditory stimuli given the activity of the neural population in AC and PPC . (B) Schematic of the discrimination being performed by the decoder, classifying sound stimuli as occuring from the left or right of the mouse. (C) Left: Mean cumulative population decoder performance (across datasets) when classifying left vs right locations, when based on "best cells", defined as individual Non-SOM cells or SOM cells with the highest decoding performance. "Best cells" and Non-SOM cells in each dataset were subsampled to match the $\mathrm{N}$ of the SOM neuron population within each imaging field of view. Right: Population decoder performance using PPC neurons. Chance performance is 0.5. (D) Mean decoder performance using all timepoints in the trial (equivalent to the final timepoints in (C) using SOM neurons, subsampled Non-SOM neurons, and "best cells" in AC and PPC. Dotted line corresponds to chance performance (50\%). (E) Mean fraction correct of cumulative sound location (left sound locations vs right sound locations) decoding in AC (right) and PPC (left) populations in high (dark) and low (light) arousal conditions. Subsampling to match arousal and sound locations was repeated 10x. (F) Cumulative fraction correct of decoding for left vs right sound location at the end of the trial in AC and PPC. Shading in C,E and errorbars in D,F indicate SEM, ${ }^{* * *} \mathrm{p}<0.001$, n.s. not significant. AC Non-SOM N=24 datasets; AC SOM N=24 datasets; PPC Non-SOM $\mathrm{N}=20$ datasets; PPC SOM N=20 datasets throughout figure panels. See Table S9 for full statistics. 
were classified as occurring during low or high arousal based on the pupil area states (Materials and Methods, 6.1, Figure $2 \mathrm{C}$ ), and low and high arousal trials were evenly balanced to train and test the decoders. Decoding performance for left vs. right sound location category using AC populations was modestly improved during heightened arousal, but decoding performance was similarly poor in low and high arousal trials using PPC populations (Figure 4E-F, Table S9). Thus, while generalized arousal seems to improve sensory coding in $A C$, it had no impact on coding within PPC populations.

To summarize our analyses characterizing the relationship between neural activity, sensory stimuli, and behavioral state, heightened arousal led to modest net increases in overall activity in AC SOM and Non-SOM populations, and more pronounced and uniform increases in activity across neurons in PPC. SOM neurons in PPC were most strongly modulated by behavioral state. Still, sensory coding only improved in AC, but not PPC, during periods of high arousal. Critically, cortical circuits are comprised of interdependent, highly interconnected neurons that share common input $(31,59-63)$, and the resulting ongoing population activity patterns interact with incoming signals related to behavioral state or sensory stimuli (64). Interactions among excitatory and inhibitory neurons, such as SOM neurons, are important for setting and maintaining the dynamics of ongoing population activity $(22,36,65)$.

In the following sections, we compare the interactions of SOM and Non-SOM neurons with each other in AC and PPC.

\section{Noise correlations were strongest within SOM populations in both AC and PPC}

Task engagement, attention, and arousal also impact neural activity at the population level, reducing shared variability in sensory cortex $(1,2,6-9,22)$. Correlated variability is higher in PPC than in AC, allowing PPC populations to encode information more stably over time, and improving readout of perceptual choices by downstream regions $(45,66)$. The local circuit mechanisms contributing to these differing population activity patterns in AC and PPC are not known, but are likely to involve inhibitory interneurons, which can modulate population activity coherence (22, 36).

The arousal-related shifts in SOM activity, especially in PPC, demonstrated here lead to the question of how SOM neuron activity co-fluctuates with other neurons in the local population. As expected from our previous work (45), pairwise correlations overall were lower in AC than in PPC $(p<.001$, Table S10). Noise correlations also tended to be weaker in the high arousal states, but the effect depended on signal correlation, area, and cell type (Figure S7, Table S11). To characterize the patterns of functional interactions within and between cell types, we measured the pairwise noise correlations between Non-SOM/Non-SOM pairs, SOM/SOM pairs, and NonSOM/SOM pairs (Figure 5A-C). In both regions, noise correlations differed across these three cell type combinations (AC: Kruskal Wallis, $p<.001$; PPC: Kruskal Wallis, $p<.001$ ). In both AC and PPC, SOM/SOM correlations were higher than both Non-SOM/Non-SOM correlations $(p<.001)$ and Non-SOM/SOM correlations $(p<.001$, Table S10). Despite the lack of monosynaptic connections among SOM neurons (26), the high SOM/SOM noise correlations in both regions support the idea of functional subnetworks of SOM neurons in local cortical circuits (67). 
The spatial scale of functional connectivity differed in AC and PPC

To characterize the spatial extent of pairwise noise correlations in AC and PPC, we related mean pairwise correlations to inter-somatic distance (Figure 5D-G). Within $100 \mu \mathrm{m}$, correlations between Non-SOM/Non-SOM pairs were similar across regions (Figure 5D-E, Table S12). However, at longer distances, AC pairwise correlations decayed rapidly, while PPC pairwise correlations remained relatively elevated across all distances (Figure 5D-F). SOM/SOM pairwise correlations remained higher in PPC than AC across all inter-somatic distances $(p<.001$, Figure $5 F$, Table S12).

To quantify the rate of decay in pairwise correlations over cortical distance, we fit an exponential equation to determine the decay constant lambda $(\lambda)$, across binned pairwise distances (Materials and Methods 7.3). The spatial decay was faster in AC than PPC for all cell type combinations (Figure 5G, $p<.001$, Table S13). The spatial patterns of noise correlations were similar when calculated based on running bout "trials" rather than sound stimulus presentations (Tables S14-16, Figure S8).

Together, the noise correlation analyses revealed both similarities and differences in the structure of functional correlations between cell types across cortex. SOM neurons tended to be more strongly correlated with each other than with other neurons in both AC and PPC. Within AC, noise correlations were highest at close pairwise distances but decayed rapidly with distance. Within PPC, pairwise correlations remained elevated across distances over $300 \mu \mathrm{m}$, with SOM-SOM correlations remaining highest across distance. These results suggest differences in the spatial scales of functional SOM subnetworks in AC and PPC (Figure $5 \mathrm{H}$ ), which may allow SOM neurons in PPC to pool excitation over overlapping, wider distances, and act as a more coordinated population in PPC than in AC (Figure $5 \mathrm{H}$ ).

\section{Functional coupling within SOM populations remained higher in both AC and PPC after discounting behavioral and sensory influences}

The noise correlation results (Figure 5) suggest important similarities and differences in the structure of functional SOM subnetworks in AC and PPC. However, pairwise noise correlations cannot easily discount the effects of ongoing fluctuations in sensory stimuli and behavioral variables such as running direction, speed, and pupil size, which modulate AC and PPC SOM and Non-SOM activity differently (Figures $1-3)(14,17,45)$. To measure correlated variability while accounting for sensory stimuli and behavioral variables, we returned to the generalized linear model (GLM) approach, to compute the level of "coupling" among SOM and Non-SOM neurons in AC and PPC in isolation of common drive from behavioral or sensory-related inputs $(45,46)$. The coupling measurement shares the goal of noise correlation analyses, while discounting many time-varying behavioral correlates simultaneously. Furthermore, coupling measurements can also measure high order co-fluctuations between multiple neurons, beyond the pairwise relationships measured with noise correlations (46).

Our approach to measuring coupling is analogous to the approach we used to determine the running velocity and pupil area contributions to each neuron's activity. We again trained and tested variants of the encoding model and compared their prediction performance of each neuron's activity. The uncoupled model included time varying predictors based on running velocity, pupil area, and sound stimulus presentation. The coupled variants of the model included 

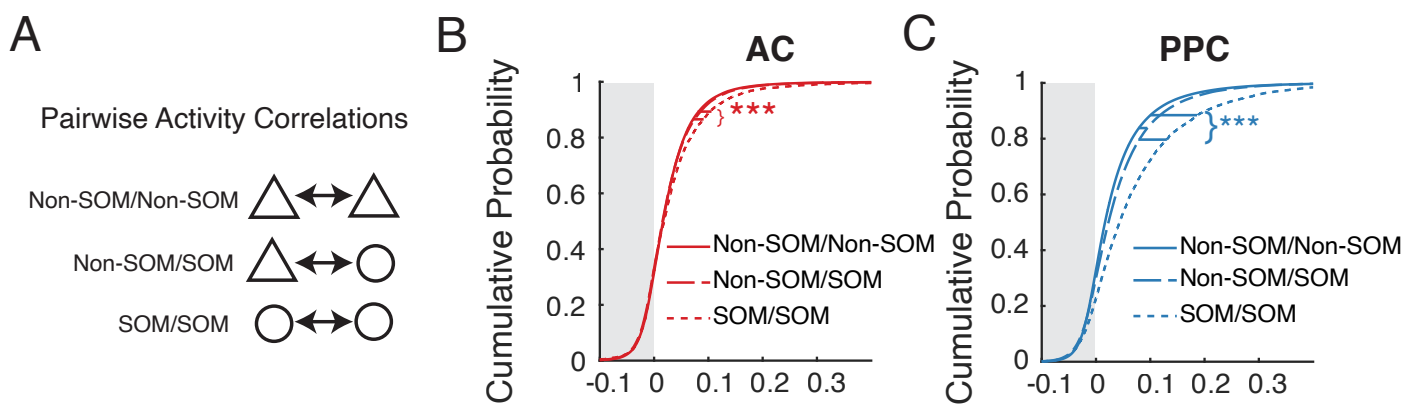

Pairwise Noise Correlation Pairwise Noise Correlation
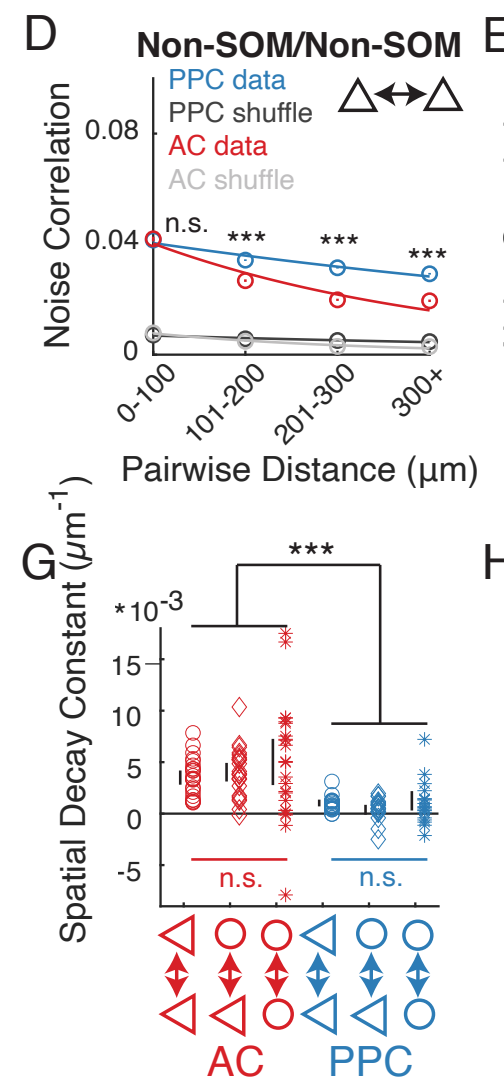

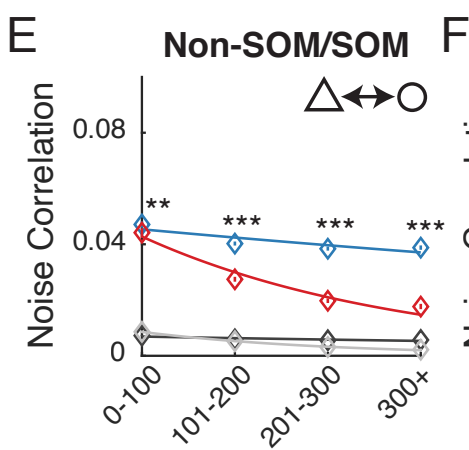

Pairwise Distance $(\mu \mathrm{m})$
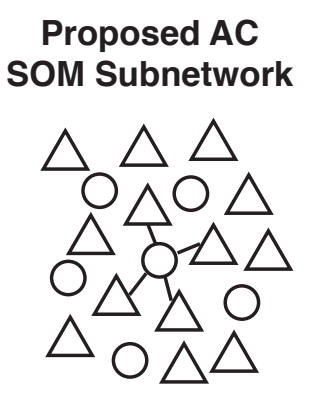

SOM/SOM

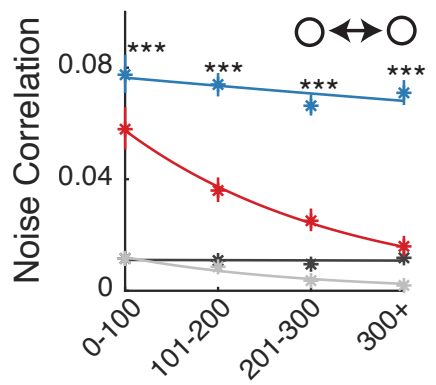

Pairwise Distance $(\mu \mathrm{m})$

\section{Proposed PPC SOM Subnetwork}

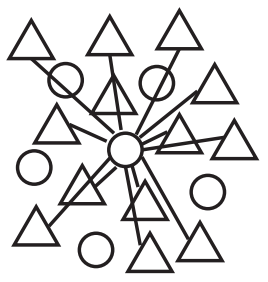

Figure 5: The Spatial Scale of Correlated Variability Differs in AC and PPC. (A) The pairwise correlations considered in following analyses. (B) Cumulative probability distributions of the noise correlations in Non-SOM/Non-SOM pairs ( $\mathrm{N}=160241)$, Non-SOM/SOM ( $\mathrm{N}=41473)$, and SOM/SOM $(\mathrm{N}=2788)$ pairs in AC. SOM/SOM correlations were higher on average than other cell type pairs. (C) As in B, for PPC neurons (Non-SOM/Non-SOM, N=620319; Non-SOM/SOM, N=129319; SOM/SOM, N=7644). Similar to AC, SOM/SOM pairs tended to be more correlated. (D) Mean pairwise noise correlations of Non-SOM/Non-SOM neurons binned by intersomatic distance in AC (red) and PPC (blue). n.s.: no significant difference; ${ }^{*} p<.05 ;{ }^{* *} p<.01 ;{ }^{* * *} p<.001$. Points indicate mean, lines are $95 \%$ bootstrapped confidence intervals of the mean. (E) As in D for Non-SOM/SOM pairs. (F) As in D-E for SOM/SOM pairs. (G) Lambda, the decay constant of a single exponential function fit to the correlation vs. distances in D-F. n.s.: No significant differences in the decay constant within regions. Each point represents the lambda values for one pair type from one dataset, black lines show 95\% bootstrapped confidence interval of the mean. AC N=24 datasets; PPC, $\mathrm{N}=20$ datasets. $(\mathrm{H})$ We propose that the spatial scale of excitatory-SOM networks is wider in PPC than in AC, explaining the differences in spatial decay of functional coupling among SOM neurons across areas. Full summary of values and statistics presented here available in Supplementary Tables 10, 12-13. 
A

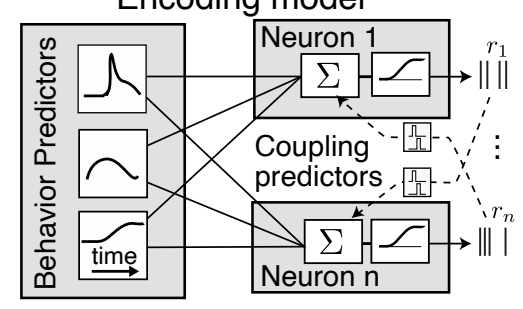

Non-SOM/Non-SOM $\triangle \longrightarrow \triangle$

Coupling: (Predict Non-SOM activity

with other Non-SOM neurons)

Non-SOM/SOM $\triangle \rightarrow 0$

Coupling: (Predict SOM activity with

SOM/SOM $\mathrm{O} \rightarrow \mathrm{O}$

Coupling: (Predict SOM activity with other SOM neurons)

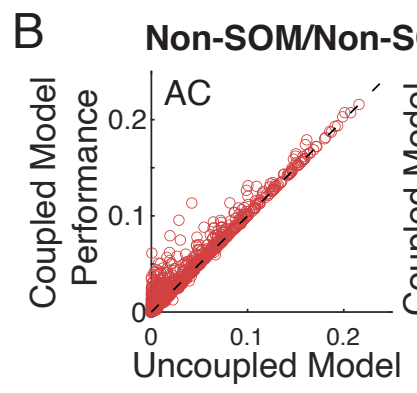

Performance

\section{C}

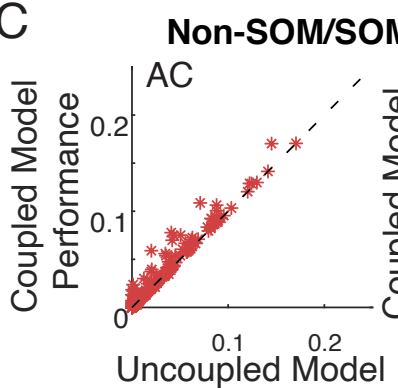

Performance

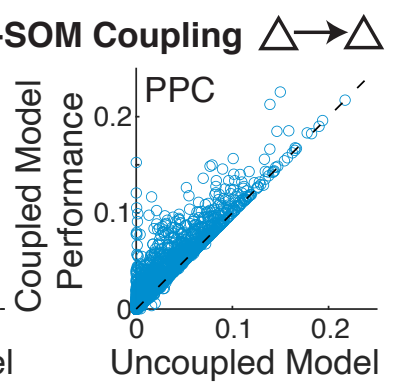

Performance

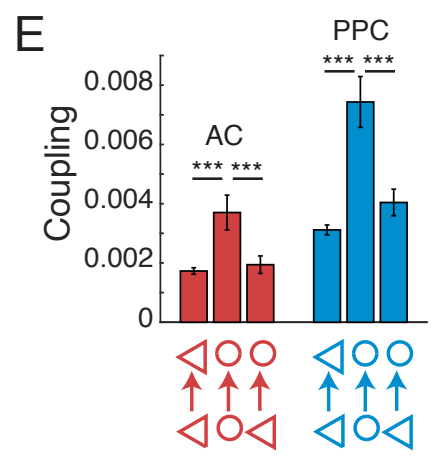

$\mathrm{F}$

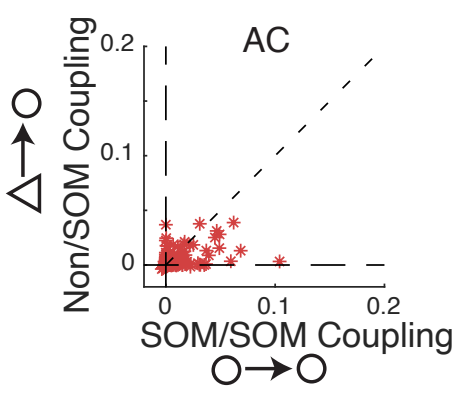

G

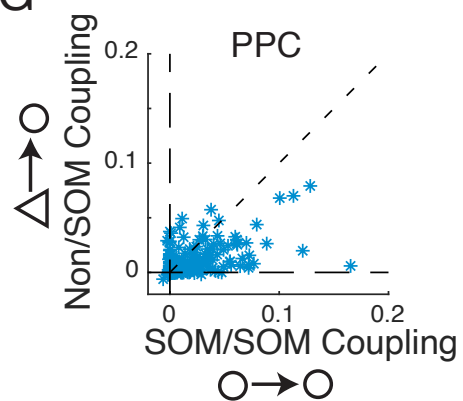

Figure 6: Functional Coupling is Highest between SOM Neurons. (A) Left: Schematic of the Generalized Linear Model (GLM) used to measure functional coupling between neuron types. Behavioral predictors and coupling predictors from either SOM or Non-SOM neurons were used to predict each neuron's activity. Right: Types of coupling assessed. (B) Performance improvement when adding Non-SOM coupling to the model to predict activity of Non-SOM neurons in AC (left, $\mathrm{N}=2428$ ) and PPC (right, $\mathrm{N}=4048$ ) neurons. $\mathrm{X}$ axis: uncoupled model prediction performance (fraction of deviance explained). $Y$ axis: coupled model prediction performance, using Non-SOM neurons as coupling predictors. (C) As in B, when using Non-SOM neurons to predict each SOM neuron's activity ( $A C, N=320$; PPC, $N=485)$. (D) As in $B-C$, when using SOM neurons to predict each SOM neuron's activity ( $A C, N=320$; PPC, $N=485$ ). (E) Mean coupling (Model performance improvement when adding coupling: Y-axis minus X-axis for each neuron in B-D), measured with the three coupled model types in AC (red bars) and PPC (blue bars) neurons. (F) Comparison of Non-SOM and SOM coupling to SOM neurons in AC. (G) As in F, for PPC SOM neurons. Error bars indicate SEM, ${ }^{* *} p<0.001$. Full summary of values and statistics in Supplementary Table 17. 

these in addition to predictors based on the activity of subsets of the simultaneously imaged neurons: the "SOM coupled model" used the activity of SOM neurons in the field of view as predictors, and the "Non-SOM coupled model" used the activity of a subsampled population of Non-SOM neurons, chosen to match the size and distance distribution of the SOM population within that field of view.

We calculated each neuron's coupling to the SOM population by subtracting the single trial prediction performance of the uncoupled model (fraction deviance explained, compared to the null model) from the prediction performance of the SOM coupled model, using data that had not been used to train the model. Similarly, each neuron's Non-SOM coupling was calculated by subtracting the performance of the uncoupled model from the performance of the Non-SOM coupled model (Materials and Methods 8.0, Figure 6A). This approach allowed us to isolate functional coupling of each neuron to local SOM and Non-SOM subpopulations that could not be explained by sound stimuli, running behavior, and pupil area fluctuations (i.e., the difference in the $y$ and $x$ axes for each neuron in Figure 6B-D).

We calculated three types of coupling: 1) Non-SOM/Non-SOM (Figure 6B); 2) Non-SOM/SOM coupling (Figure 6C); and 3) SOM/SOM coupling (Figure 6D). As expected from previous results (Runyan et al., 2017), coupling among Non-SOM neurons was higher in PPC than AC $(p<0.001$, Figure 6E). Coupling among SOM neurons was also higher in PPC than in AC $(p<0.001)$, as was Non-SOM/SOM coupling ( $p<0.001)$. Consistent with noise correlations (Figure 5A-C), coupling among SOM neurons was higher than coupling between other cell types in both $A C$ and PPC $(p<0.001$, Figure 6E-G, Table S17).

In summary, even when accounting for co-modulation of neural activity by changes in running speed, pupil size, and sound stimulation, coupling among neurons was high in PPC relative to $\mathrm{AC}$, and coupling among SOM neurons was higher than coupling among other cell types within both regions.

\section{Discussion}

To compare the functional structure of inhibitory subnetworks across cortex, we have measured the spike-related activity in populations of somatostatin-expressing inhibitory interneurons (SOM) and Non-SOM neurons in auditory cortex (AC) and in posterior parietal cortex (PPC), while mice experienced spontaneous arousal shifts. We examined the impact of these shifts on ongoing activity patterns, as well as on sound-evoked responses. Our results reveal both common principles in the functional structure of SOM and Non-SOM population activity, as well as regionspecific differences, implying some specialization in the function of SOM neurons across sensory and association-level cortices.

In AC, where spontaneous population activity was decorrelated overall compared to PPC, the effects of arousal on individual neurons were diverse, with only a small net positive effect on population activity. In PPC, increases in arousal led to a general increase in activity across most neurons. In AC, SOM and Non-SOM populations showed similarly diverse arousal-related responses, while in PPC, SOM neurons were even more strongly activated with increased arousal than Non-SOM neurons. Although increases in running speed and pupil size were strongly related to each other, we were able to disentangle the contributions of running velocity and pupil size to the activity of individual neurons in AC and PPC using an encoding model (Figure 3 ). In both regions, pupil size and running velocity contributed to the activity of SOM and 
386 Non-SOM neurons. However, more of the arousal-related activity modulation in PPC was explained by changes in running velocity, while in $A C$, pupil size and running velocity were similarly weak contributors to activity. This is likely related to PPC's role in navigation and sensorimotor transformations $(45,47,51-53,57,68)$.

390

The effects of arousal on neural activity and coding have been examined in depth across primary sensory cortices. Overall, our results are consistent with this body of work, which has revealed a relationship between correlated variability and arousal state (6-9). In primary auditory cortex, the Yerkes-Dodson (inverted-U) relationship between pupil size and sound decoding has been observed $(9,14)$, which aligns well with the known links between norepinephrine levels and behavioral performance and an "optimal" level of arousal for behavioral performance (69). We did not observe this effect, instead measuring a modest improvement in decoding accuracy with arousal. Mice in our experiments were most likely to visit two separable behavioral states (stationary/unaroused or running/aroused), without the gradation of different levels of arousal observed by others. Our "high" arousal state likely includes a mixture of the moderate and high arousal states defined by others when three separable states can be identified. It is important to note that while locomotion, whisking, and pupil dilations have all been considered as behavioral correlates of an animal's arousal state, motor-related feedback acts on specialized circuits within visual, somatosensory, and auditory cortices $(11,17,18,70-72)$ that interact with neuromodulatory inputs, leading to different arousal-related effects on network activity in those regions. It is unlikely that any aroused state is without a related change in fidgeting, facial movements, or other motor outputs $(73,74)$.

In general, activity within the SOM subpopulation was more coordinated in both AC and PPC, compared to the Non-SOM population, which includes mostly excitatory neurons. This is consistent with previous findings within primary visual cortex, in which shared variability is overall higher within inhibitory cell types than between types $(67,75,76)$. Our similar results in sensory and association cortex indicate it is likely a general property of the somatostatin population across cortical areas, despite differences in overall correlated variability between the two regions. Despite this similarity in cell-type specific activity relationships in AC and PPC, the spatial scales of functional interactions between neurons differed markedly in the two regions. In AC, pairwise correlations decayed rapidly with distance (Figure 5), as has been well established in sensory cortex (77-82). In contrast, pairwise noise correlations remained high over longer distances in PPC, suggesting a different spatial structure of correlated variability in association cortex, consistent with findings in primates (83). SOM/SOM noise correlations, which were stronger overall than Non-SOM/Non-SOM correlations, remained high across all measured distances in PPC. Given the role for SOM neurons in providing lateral inhibition by pooling excitatory inputs and inhibiting neighboring neurons $(33,84)$, we suspect that the wider spatial scale of SOM/SOM activity correlations in PPC implies that SOM neurons receive excitatory synapses from farther distances in PPC than in AC (Figure 5H). Future work will determine whether this is the case, and whether the magnitude and spatial scale of correlated variability, particularly within SOM subnetworks, increases systematically across the cortical hierarchy.

Taken together, our results demonstrate key differences in the spatial extent of coordinated inhibitory subnetworks across cortex, as well as in arousal-related modulation of activity dynamics, adding to a growing body of evidence that cortical microcircuits are functionally specialized across cortical regions $(39,40,45)$. The role of distinct cortical areas in processing incoming sensory information or generating behaviors varies across cortex, and the underlying 
spatial patterns and dynamics of population activity may be tailored to these specialized roles. Primary sensory areas, such as AC, encode fine-grain details time-varying sensory stimuli, and are organized topographically. Meanwhile, multisensory inputs converge in association-level regions such as PPC, which then flexibly integrates sensory information over time and transforms it to influence behavioral choices $(45,57,58,85,86)$. The flexible, task-dependent integration of diverse feedforward signals in PPC may require larger scale network dynamics, as we observed in SOM and Non-SOM populations.

\section{Materials and Methods}

\subsection{Statistics}

All pairwise comparisons were done with two-sided paired or unpaired permutation (i.e. randomization) tests with 10,000 iterations as indicated, where $p<.0001$ indicates the highest significance achievable given the number of runs performed. All permutation tests were performed for differences in means. For statistical comparisons involving more than two groups, we used Kruskal-Wallis (non-parametric ANOVA) and used unpaired permutation tests post-hoc to determine which groups differed from each other. Data fell into natural groupings by 1 ) brain area (AC or PPC) and by 2) cell-type (SOM or Non-SOM), as indicated by expression of the red fluorophore, tdTomato. All bar plots show mean and bootstrapped $95 \%$ confidence intervals unless otherwise indicated. When multiple comparisons were made between groups, $p$ values were Bonferroni-corrected. Sample sizes were chosen based on previous studies comparing population activity dynamics across brain areas or cell types $(45,76)$.

\subsection{Animals}

All procedures were approved by the University of Pittsburgh Institutional Animal Care and Use Committee. Homozygous SOM-Cre mice (Sst-IRES-Cre, JAX Stock \#013044) were crossed with homozygous Ai14 mice (RCL-tdT-D, JAX Stock \#007914) obtained from Jackson Laboratory, ME, USA, and all experiments were performed in the F1 generation, which expressed tdTomato in $\mathrm{SOM}+$ neurons. Mice were group housed in cages with between 2 and 4 mice. Adult (8-24 weeks) male and female mice were used for experiments ( 4 male, 2 female). Mice were housed on reversed $12 \mathrm{hr}$ light/dark cycle, and all experiments were performed in the dark (active) phase.

\subsection{Surgical procedures}

Mice were anesthetized with isoflurane ( $4 \%$ for induction, and 1-2\% maintenance during surgery), and mounted on a stereotaxic frame (David Kopf Instruments, CA). Ophthalmic ointment was applied to cover the eyes (Henry Schein, NY). Dexamethasone was injected 12-24 hours prior to surgery, and carprofen and dexamethasone (Covetrus, ME) were injected subcutaneously immediately prior to surgery for pain management and to reduce the inflammatory response. Two $2 \times 2 \mathrm{~mm}$ craniotomies were made over left AC (centered at $3.0 \mathrm{~mm}$ posterior and $4.3 \mathrm{~mm}$ lateral to bregma) and PPC (centered at $2 \mathrm{~mm}$ posterior and $1.75 \mathrm{~mm}$ lateral to bregma). $1-4$ evenly spaced $\sim 60 \mathrm{nl}$ injections of AAV1-synapsin-I-GCamp6f (Addgene, MA stock \#100837) that had been diluted to a titer of $\sim 1 \times 10^{\wedge} 12 \mathrm{vg} / \mathrm{mL}$ using sterile PBS were made in each cranial window, centered on the same coordinates listed above. A micromanipulator (QUAD, Sutter, CA) was used to target injections $\sim 250 \mu \mathrm{m}$ under the dura at each site, where $\sim 60 \mathrm{nl}$ virus was pressureinjected over 5-10 minutes. Pipettes were not removed until 5 minutes post-injection to prevent backflow. Dental cement (Parkell, NY) sealed a glass coverslip (3mm) over a drop of Kwik Sil 
474 (World Precision Instruments, FL) over the craniotomy. Using dental cement, a one-sided titanium

475 headplate was attached to the right hemisphere of the skull. After mice had recovered from the

476 anesthesia, they were returned to their home cages, and received oral carprofen tablets (Bio-

477 Serv, NJ) for 3 days post-surgery.

$478 \quad 3.0$ Experimental Setup

\section{$479 \quad 3.1$ Two-photon microscope}

480 Images were acquired using a resonant scanning two-photon microscope (Ultima Investigator, 481 Bruker, $\mathrm{WI}$ ) at a $30 \mathrm{~Hz}$ frame rate and 512 x 512 pixel resolution through a 16x water immersion 482 lens (Nikon CF175, 16X/0.8 NA, NY). On separate days, either AC or PPC was imaged at a 483 depth between 150 and $300 \mu \mathrm{m}$, corresponding to layers $2 / 3$ of cortex. For AC imaging, the 484 objective was rotated 35-45 degrees from vertical, and for PPC imaging, it was rotated to 5-15 485 degrees from vertical, matching the angle of the cranial window implant. Each field of view contained approximately 100-300 neurons total, and 5-30 SOM neurons. Excitation light was provided by a femtosecond infrared laser (Insight X3, Spectra-Physics, CA) tuned to $920 \mathrm{~nm}$. Green and red wavelengths were separated through a $565 \mathrm{~nm}$ lowpass filter before passing through bandpass filters (Chroma, ET525/70 and ET595/50, VT). PrairieView software (v5.5, Bruker, $\mathrm{Wl}$ ) was used to control the microscope.

492 Running velocity was monitored on pitch and roll axes using two optical sensors (ADNS-98000, 493 Tindie, CA) held adjacent to the spherical treadmill. A microcontroller (Teensy, 3.1, Adafruit, NY) communicated with the sensors, demixing their inputs to produce one output channel per rotational axis using custom code. Outputs controlling the galvanometers were synchronized with running velocity using a digital oscilloscope (Wavesurfer, Janelia, VA).

Pupil images were acquired at $1280 \times 1024$ pixels, at $10 \mathrm{~Hz}$ from an infrared (IR) camera focused on one eye (Flea3 FL3-U3-13Y3M-C 1/2" Monochrome USB 3.0 Camera, 1.0x SilverTL Telecentric Lens, FOV $=6.74 \mathrm{~mm} \times 5.39 \mathrm{~mm}$, Edmund Optics, NJ). The pupil was illuminated by the IR light emitted by the two-photon laser and required no additional IR illumination. Movies were acquired with Matlab Image Acquisition Toolbox (Mathworks, MA). Pupil area was determined in each pupil movie frame post-hoc using custom Matlab code (Mathworks, MA). The pupil was constricted by controlling ambient illumination with an array of LCD screens (LG LP097QX1, South Korea), to maintain a moderate pupil area baseline from which increases and decreases could be measured.

Imaging began 3-5 weeks post-surgery once robust expression of the GCaMP6f virus was observed. Imaging sessions lasted 60-90 min total, divided in half between spontaneous activity and passive listening (ordering was random), and alternated between AC and PPC across days. Fields of view were selected based on co-expression of viral GCaMP6f (all neurons) and transgenic tDTomato (SOM neurons). Multiple imaging sessions were performed in each cranial window, focusing slightly different depths and lateral/posterior locations within the imaging windows across sessions. AC and PPC were each imaged in six mice (biological replicates). Each cranial window was imaged up to 11 times (technical replicates). Imaging from a given 
cranial window was suspended when we observed nuclear inclusion in 2 or more cells in the field of view, which indicates an over-expression of GCaMP6f. In each imaging session, GCaMP6f fluorescence changes were imaged in SOM (tDTomato+) and Non-SOM neurons, while mice ran freely on a spherical treadmill. In the "spontaneous context", no sensory stimuli were delivered, while in the passive listening context, location-varying sound stimuli were presented. Spontaneous and passive listening contexts lasted $\sim 25-50$ minutes each. The order in which the contexts occurred was random.

\subsection{Sound stimuli}

Either immediately before or following spontaneous activity context, the same field of view was imaged for 25-50 minutes during passive listening. Four magnetic speakers were positioned in a semicircular array, centered on the mouse's head (MF1-S, Tucker-Davis, FL). The speakers were positioned at $-90,-30,+30$ and +90 degrees in azimuth and driven by digital/analog converter (National Instruments). Speakers were calibrated to deliver similar sound levels ( $70 \mathrm{db})$ in a sound isolation chamber using a microphone at the same distance from the speakers as the mouse's head using a random incidence microphone (4939, Brüel \& Kjær, Denmark). During passive listening, 1 or 2 second dynamic ripples (broadband stimuli created in Matlab by summing 32 tones spaced across $2-32 \mathrm{kHz}$, which fluctuated at $10-20 \mathrm{~Hz}(87)$ ) were presented from one of eight locations. Four of the sound locations corresponded to the locations of the four speakers $(-90,-30,+30,+90$ degrees $)$, while the other four sound locations $(-60,-15,+15,+60$ degrees) were simulated using vector based intensity panning, where the same sound stimulus was delivered to two neighboring speakers simultaneously, scaled by a gain factor (45). Dynamic ripples were chosen to optimally drive populations of neurons in auditory cortex with diverse frequency tuning preferences. Each sound repeated three times at one location before switching to another. Each ripple would play from all eight locations in randomized order continuously throughout the session with a $240 \mathrm{~ms}$ gap between each sound. Output controlling the audio speakers was aligned with two-photon imaging and running velocity offline using Wavesurfer (Janelia, VA).

\subsection{Data Processing}

Imaging datasets from $24 \mathrm{AC}$ fields of view and 20 PPC fields of view were included from 6 mice. We excluded any datasets where we observed significant photobleaching or filled cells (see above). We excluded any AC or PPC dataset from analysis if fewer than $1 / 3$ of neurons that were significantly responsive (according to our definition in section 5.1) to at least one sound location, as we were interested in the effect of arousal on both spontaneous and sound-evoked responses. For AC datasets, we analyzed single-cell responses to pure tones on a subset of fields of view from each mouse, and then anatomically aligned all fields of view from datasets collected from each window, to ensure each field of view lay in a region representing tone frequencies in the sonic range of the tonotopic axis of primary auditory cortex. We eliminated any datasets where $>50 \%$ of tone-responsive neurons had a preferred frequency was in the ultrasonic range $(>20 \mathrm{kHz})$, as well as any fields of view that were aligned anterior to a field of view where this was observed, to assure that we were seeing sound responses in the range of frequencies primarily represented by our dynamic ripples (described in section 3.4). We collected widefield fluorescence responses to pure tones in all AC cranial windows and observed ample pure tone responses in the sonic range for all $\mathrm{AC}$ windows; however, the extent of the viral expression within windows was too spatially limited to allow for mapping of specific regions. 


\subsection{Image Processing}

For each field of view, the raw calcium movies collected during the spontaneous activity and passive listening contexts were concatenated for motion correction, cell body identification, and fluorescence and neuropil extraction. These processing steps were performed using Suite2p 0.9.3 in Python (88). Suite2p first registered images to account for brain motion, and clustered neighboring pixels with similar time courses into regions of interest (ROIs). ROIs were manually curated using the Suite2p GUI, to ensure that only cell bodies as opposed to dendritic processes were included in analysis, based on morphology. Cells expressing tdTomato (SOM cells), were identified using a threshold applied in the Suite2p GUI based on mean fluorescence in the red channel after bleed-through correction applied by Suite2p's cell detection algorithm, along with manual correction. For each ROI, Suite2p returned a raw fluorescence timeseries, as well as an estimate of neuropil fluorescence that could contaminate the signal. For each cell, we scaled the neuropil fluorescence by a factor by 0.7 and subtracted this timeseries from the ROl's raw fluorescence timeseries to obtain a neuropil-corrected fluorescence signal for each selected cell.

\section{2 $\Delta F / F$ and deconvolution}

Once the neuropil corrected fluorescence was obtained for each neuron, we calculated $\Delta \mathrm{F} / \mathrm{F}$ for each cell in each frame by calculating $\left(F-F_{\text {baseline }}\right) / F_{\text {baseline }}$ for each frame, where $F$ is the fluorescence of a given cell at that frame and $F_{\text {baseline was the eighth percentile of that cell }}$ spanning 450 frames before and after ( $15 \mathrm{~s}$ each way, $30 \mathrm{~s}$ total). $\Delta \mathrm{F} / \mathrm{F}$ timeseries were then deconvolved to estimate the relative spike rate in each imaging frame using the OASIS toolbox (89). We used the AR1 FOOPSI algorithm and allowed the toolbox to optimize the convolution kernel, baseline fluorescence, and noise distribution. A threshold of .05 a.u. was applied to remove all events with low magnitude from deconvolved activity timeseries. All analyses were performed with both $\Delta F / F$ and deconvolved activity and showed the same trends, unless otherwise indicated. Outside of Figure $1 \mathrm{~F}$ and $1 \mathrm{H}$ only results using deconvolved activity are shown.

\subsection{Single cell modulation}

\subsection{Sound responsiveness}

Sound responsiveness was calculated based on the mean z-scored deconvolved activity of each neuron aligned on sound onset. For each neuron, we calculated the difference between the mean activity during the sound presentation at a certain location (either 1 or 2 seconds) and the mean activity in the 240 milliseconds prior to sound onset. We calculated sound responsiveness separately for each sound location as the mean difference in activity between these two windows for each neuron. We then compared the observed sound responsiveness of each neuron for each sound location to a shuffled distribution. Each cell's activity was shifted randomly by at least 5 seconds in time relative to sound location time series, and for 1000 time-shifted iterations, sound responsiveness to each sound location was recalculated. Each sound responsive neuron had a positive sound responsiveness value for at least one location that was greater than the $95^{\text {th }}$ percentile of that cell's shuffled distribution for that location. All other neurons were not considered sound responsive. 


\subsection{Running bouts and modulation}

Running bout onsets were defined as transitions in speed from below $10 \mathrm{~cm} / \mathrm{s}$ to above $10 \mathrm{~cm} / \mathrm{s}$, and required that the mean running speed in the $1 \mathrm{~s}$ following the transition was three times greater than the $1 \mathrm{~s}$ prior to running bout onset, and that the mouse maintained a speed of a minimum of $15 \mathrm{~cm} / \mathrm{s}$ for the following two seconds.

Running modulation was calculated as the difference in mean z-scored deconvolved activity of a cell in the $1 \mathrm{~s}$ prior to running bout onset and the mean activity of a cell in the $3 \mathrm{~s}$ window following running bout onset. A shuffling procedure was applied to determine which cells were positively, negatively, and not modulated by running. Each cell's activity was shifted randomly by at least 5 seconds in time relative to running speed timeseries, and for 1000 time-shifted iterations, running modulation was recalculated. Positively modulated neurons had positive running modulation values higher than the $95^{\text {th }}$ percentile of that cell's shuffled distribution. Negatively modulated cells had negative running modulation values lower than the $5^{\text {th }}$ percentile of that cell's shuffled distribution. All other cells were considered to be not modulated by running speed increases.

\subsection{Pupil dilation events and modulation}

Pupil area was normalized to its maximum across the imaging session. To identify pupil dilation events, we first identified all local maxima of the pupil area. We then found the point prior to this where the derivative of pupil area was zero. We included events where the change from the inflection point to the local maximum was at least a $40 \%$ increase in pupil area and that the change from inflection point to local maximum was less than 1 second, and that the local maximum was at least $50 \%$ of the maximum total area by the pupil during that imaging session. We considered each inflection point to be the onset of dilation events.

To capture all pupil dilation related activity, which has a slower time course than running (Figure S2), we calculated pupil modulation as the difference between the mean z-scored deconvolved activity in the 1-s time window prior to dilation event onset and the mean activity in the 5-s time window following dilation event onset. We applied the same shuffling procedure described in the section above to determine which neurons were positively, negatively, and not modulated by pupil dilation events.

\subsection{Arousal Clustering}

\subsection{Low and high arousal based on pupil area}

K-means clustering was applied to the full pupil area timeseries, which included both spontaneous activity and passive listening contexts, to classify each pupil area value as low, transitional, or high arousal. Each pupil area timeseries was mean normalized using the following equation:

$$
\frac{x-\bar{x}}{\bar{x}}
$$

The Manhattan (aka City Blocks) distance metric was applied to define 2 centroid clusters that served as the high and low arousal groups. Transition periods included points whose absolute difference in distance to the high and low arousal centroids was less than 0.05 . 


\subsection{Arousal Modulation Index}

Arousal modulation index was calculated for each neuron using the following equation:

$$
\frac{F R_{h i}-F R_{l o}}{F R_{h i}+F R_{l o}}
$$

For FR, we first maximum-normalized the deconvolved activity trace of each neuron. For FRhi, we summed the maximum-normalized deconvolved activity during frames in the "high" cluster and divided that by the total time spent in the high cluster during either the spontaneous (Figure 2) or passive (Figure S3). FR เo was determined similarly, using the low arousal cluster. This index allowed a modulation index between -1 and 1 , where negative values indicated higher activity in the low arousal cluster, and positive numbers indicated higher activity during the high arousal cluster.

\subsection{Noise Correlations}

\section{Pairwise Correlations 7.1}

Pairwise noise correlations were calculated based on trial-to-trial fluctuations around mean sound-evoked responses. One sound trial included three repeats of a given sound stimulus. We calculated mean sensory-evoked activity for each neuron at each location and binned the activity of each neuron by 10 frames ( $\sim 330$ milliseconds) over the course of the sound trial. Trials were either 3 or 6 seconds long depending on the duration of the dynamic ripple. Mean responses were calculated separately for different durations at the same location. For each neuron, we subtracted the mean sensory response for each location at each trial, and then concatenated these mean-subtracted trial responses. For each pair of neurons, we computed the Pearson correlation coefficient between these binned, mean-subtracted activity timeseries. We also calculated a shuffled distribution of pairwise noise correlations by shuffling the identity of trials for each neuron independently within sound location/duration condition and then computing the Pearson correlation coefficient in the same way. We also computed signal correlation between each pair of neurons, as the Pearson correlation between the pair of neurons' mean soundevoked responses to each sound duration/location after they had been binned into 10 frame ( 330 milliseconds) bins and concatenated.

During the spontaneous context, a similar approach was used to find running-based noise correlations. The three seconds following each running bout onset was defined as a "trial", and the Pearson correlation was computed based on the binned ( $330 \mathrm{~ms})$ activity of pairs of neurons after subtracting mean running-related activity from each neuron's timeseries.

\subsection{Noise correlations in low and high arousal clusters}

Because it was rare for a mouse's pupil to remain in either the high or low arousal cluster for the entire 3-6 s duration of a trial based on all three repetitions of a sound location, we instead focused this analysis on first of the three sound stimulus repeats within a trial. We defined a sound trial as "high arousal" if $90 \%$ of imaging frames collected during that sound presentation were classified within the high arousal cluster (Materials and Methods 6.1), and as "low arousal" if $90 \%$ of imaging frames collected during that sound presentation were classified within the low arousal cluster. As before, we binned each neuron's activity in 10 frame (330 ms) bins. For each 
678 trial classified as high or low arousal, each cell's the mean sound response to all matching sound 679 location/duration first repetition presentations (including trials belonging to low, high, or neither cluster) was subtracted. Because the ratio of low and high arousal trials was different across days for different mice, we balanced the number of sound location trials by a sub-sampling procedure, where for 10 iterations a random subset of trials was selected from the arousal cluster that had more trials to match the number of trials of the rarer arousal cluster. We only included data from sessions where there were at least 100 first-repeat trials balanced for sound location and duration in both the high and low arousal cluster, and where there were at least 5 trials representing each sound location in both low and high arousal. Within each arousal cluster (low and high), the activity traces.

\subsection{Correlation decay over distance}

690 We considered pairs of neurons in four separate distance bins, pairs of neurons within 0-100, 691 100-200, 200-300, 300+ $\mu \mathrm{m}$ from each other. For all neuron pairs, as well as by dataset, we fit 692 decay constant $\lambda$ based on the mean noise correlations of pairs in each bin using the following 693 equation:

Where $\rho$ is the pairwise noise correlation between cells, and $\mathrm{I}$ is a decay constant that describes the rate of decay over distance $(\mu \mathrm{m})$. We calculated this decay constant, I, using noise correlations based on trial-to-trial fluctuations centered either on mean sound-location related responses during the passive listening context (Figure 5G), or on mean running bout related responses during the spontaneous context (Figure S8D). This decay constant, I, was calculated considering the means of each distance bin using all pairs from all datasets, as well as for each dataset considered separately.

703 Using Generalized Linear Models (GLMs), the time-dependent effects of all measured external 704 variables and activity of neighboring neurons on each neuron's activity were estimated $(45,46)$. 705 For this approach, several classes of predictors were used in different combinations: running, 706 pupil, sound location, and activity of SOM or Non-SOM neurons. We used a Bernoulli-based GLM 707 to weight various combinations of predictors based on these variables to predict each neuron's 708 binarized activity (timeseries of relative spike rates were thresholded at 0.05 ). The encoding 709 model is fully described in our previous work (45)

\subsection{Pupil and Running Predictors}

711 Running velocity was measured at a higher time resolution than imaging and was binned to 712 match the sampling rate of two-photon images $(30 \mathrm{~Hz})$. We included the velocity along the pitch 713 and roll axes of the treadmill (relative to the mouse's body axis). Running velocity measurements 714 were separated into four channels, (1) forward, (2) reverse, (3) left, (4) right directions based on 715 rotation along these axes. Running velocity changes could both precede or follow activity of 716 individual neurons, so time series of running velocity were convolved with four evenly spaced 717 Gaussian basis functions (240 milliseconds half-width at half-height) extending $1 \mathrm{~s}$ both forward 
718 and backward in time (8 basis functions total for each running direction: forward, reverse, left, and

719 right, Figure S6). Changes in pupil area were modeled similarly. Because pupil is a slower signal,

720 the pupil area trace was convolved with 16 evenly spaced Gaussian basis functions 4s forward

721 and backward in time to allow either prediction or response to pupil area changes.

\subsection{Sound Stimulus Predictors}

723 For sound stimulus onsets at each of the possible sound locations, 12 evenly spaced Gaussian

724 basis functions (170 millisecond half width at half height) extended $2 \mathrm{~s}$ forward in time from each

725 sound onset. First, second, and third repeats were represented separately due to potential

726 adaptation-related effects. This resulted in 12 basis functions per repeat per sound location $\times 3$

727 repeats $\times 8$ locations for 288 sound predictors.

\section{$728 \quad 8.3$ Coupling Predictors}

729 For a given neuron being fitted, the relative spike rate of all individual SOM neurons as well as

730 their activity averaged across cells was convolved with two boxcar functions extending $\sim 66$

731 milliseconds forward in time from the activity of the predictor neurons (each boxcar was non-zero

732 for only a single imaging frame or $\sim 33$ milliseconds). Because in all datasets there were more

733 Non-SOM neurons than SOM neurons, to estimate Non-SOM coupling, a separate set of Non-

734 SOM predictors was randomly selected for each neuron to match the population size and

735 distance of the SOM neurons from that neuron. Creating these separate sets of predictors

736 allowed us to compare functional coupling within and between the SOM and Non-SOM

737 populations within each field of view.

\subsection{GLM fitting procedure}

739 All predictors were maximum normalized before the fitting procedure. Beta coefficients for the 740 predictors were fitted to each neuron's activity individually, using the glmnet package in $\mathrm{R}(90)$ 741 with elastic-net regularization, which smoothly interpolated between $L_{1}$ and $L_{2}$ type regularization

742 according to the value of an interpolation parameter $\alpha$, such that $\alpha=0$ corresponded to $L_{2}$ and $\alpha=1$

743 corresponded to $L_{1}$. We selected $\alpha=.25$.

744 The GLMs were fit separately for the spontaneous and passive listening contexts. Within each 745 context, trials were randomly split into training ( $70 \%$ of trials) and test (remaining $30 \%$ of trials) sets, while balancing the distribution of sound locations. Fitting was performed on the training set, and within each training dataset, cross validation folds $(3 \mathrm{x})$ were also pre-selected so that sound locations were evenly represented. Model performance (see below, 8.5) was assessed on the test set. Each model was thus fitted and tested on entirely separate data to prevent over-fitting concerns. This train/test procedure was repeated ten times, with random subsamples of the data included in train and test segments. Each model's overall performance was assessed as its mean across all 10 iterations.

\subsection{GLM model performance}

754 Each model's performance for each cell was defined as the fraction of explained deviance of 755 each model (compared to the null model). In the null model, only a constant (single parameter) 756 was used to fit the neuron's activity and no time-varying predictors were included. We calculated 757 the deviance of the null and behavior and coupling model variants. For each model, the fraction of 
758 null model deviance explained by the model was then calculated ((null deviance - model

759 deviance)/null deviance). Deviance calculations were performed on a test dataset (30\% of the

760 data), which had not been included in the fitting procedure, and this train/test procedure was

761 repeated ten times on randomly subsampled segments of the data.

762

763

764

765

766

767

768

769

770

771

772

773

774

775

776

777

778

779

780

781

782

783

784

785

786

787

788

\subsection{Running and pupil contribution}

While running and pupil area fluctuations are highly correlated, they have separable influences on neural activity (6). To identify the separate contributions of running and pupil area on SOM and Non-SOM neurons, we fit three separate models: (1) full behavior model, (2) "no pupil" model, (3) "no running" model. In the full behavior model, all running, pupil, and sound predictors were included to fit each neuron's activity. The "no pupil" model did not include the pupil predictors, and the "no running model" did not include the running predictors.

By comparing the model performance (fraction deviance explained, see above 8.5) in the full vs. no pupil or no running models, we estimated the contribution of pupil or running to a neuron's activity, that could not be compensated for by the other variable. Running contribution was calculated as the difference in fraction deviance explained of the full model and fraction deviance explained of the no-running model $\mathrm{d}_{\mathrm{fb}}-\mathrm{d}_{\mathrm{hr}}$. Pupil contribution was calculated as the difference in fraction deviance explained of the full model and fraction deviance explained of the no-pupil model $d_{f b}-d_{n p}$.

\subsection{Coupling}

We previously demonstrated that by comparing the model performance (fraction of explained deviance) in the coupled model to the performance of the uncoupled model, we could estimate the level of correlation between a given neuron and the neurons in the simultaneously imaged population, while considering the contributions of behavior and stimulus-related variables (45).

Here, we computed several types of "coupling" by comparing the model performance (fraction deviance explained) of each coupled variant of the model to the behavior-only model. We computed three types of coupling: (1) Non-SOM/Non-SOM coupling, (2) SOM/SOM coupling, (3) Non-SOM/SOM coupling. Each type of coupling was computed in the same way, as the difference between the fraction deviance explained of the full behavior model and the fraction deviance explained of a coupled model using other neurons as predictors. However, for each type of coupling, there was a different combination of the type of cell being fit and the type of predictors being used to fit that cell, as follows:

1) Non-SOM/Non-SOM coupling: Defined as the improvement in model performance when using Non-SOM predictors to explain Non-SOM activity, compared to the behavior-only model, dNon-SOM-dbehav

2) SOM/SOM coupling: Defined as the improvement in model performance when using other SOM predictors to explain SOM activity, compared to the behavior-only model, dsom-dbehav

3) Non-SOM/SOM coupling: Defined as the improvement in model performance when using Non-SOM predictors to explain SOM activity, compared to the behavior only model, $\mathrm{d}_{\text {Non- }}$ som-dbehav.

These various types of coupling allow a characterization of the functional relationships among distinct classes of neurons across cortical areas. 


\subsection{Decoding}

The details of the decoder that we built to estimate the information in AC and PPC populations about sound stimulus have been previously described (45). Briefly, for each trial we decoded sound stimulus location from single-trial population activity by computing the probability of external variables (sound location Left/Right category, or specific sound location) given population activity. We used Bayes' theorem and population response probabilities estimated through the behavior-only (uncoupled) GLM and its predictors in that trial to compute the posterior probability of each possible sound location stimulus. The decoder was "cumulative" in time, as for each time point $t$, it was based on all imaging frames from the initiation of the trial time $t$. The decoded stimulus location at each time $t$ was defined as the stimulus location with the maximum posterior probability, based on individual neurons or on a population of simultaneously imaged neurons. The population could include SOM, Non-SOM, or the "best" neurons. Non-SOM neurons were randomly subsampled 10 times, matching the sample size of SOM neurons in each iteration. The "best" neurons were selected as the $\mathrm{n}$ individual neurons with the best decoding performance, where $n$ is the number of SOM neurons simultaneously imaged. Decoder performance was calculated as the fraction of correctly classified trials at each time point.

To compare decoder performance in low and high arousal states, trials were classified as "low" or "high" arousal based on normalized pupil area (see section 7.2). Only the first sound repeat of each trial was used. Trials were randomly subsampled in the test set to ensure an even distribution of low and high arousal trials, and sound locations. This random subsample was repeated ten times.

\subsection{Sound location selectivity}

To assess the selectivity of sound-related activity in SOM and Non-SOM neurons in AC and PPC, trial-averaged responses were used to calculate the "location selectivity index", based on vector averaging in the preferred sound direction:

$$
L S I=\sqrt{\left(\sum_{i=1}^{n} R\left(\Theta_{i}\right) \cos \left(2 \Theta_{i}\right)^{2}+\sum_{i=1}^{n} R\left(\Theta_{i}\right) \sin \left(2 \Theta_{i}\right)^{2}\right)} / \sum_{i=1}^{n} R_{i}
$$

where $R$ is the average response during the sound location presentation, and $\Theta$ is the sound location from -90 to +90 degrees, indexed by I $=1$ to $n(8)$. LSI can vary continuously from 0 (unselective) to 1 (selectively responding to only one sound location).

\subsection{Histology}

After all imaging sessions had been acquired, each mouse was transcardially perfused with saline and then $4 \%$ paraformaldehyde. The brain was extracted, cryoprotected, embedded, frozen, and sliced. Once slide mounted, we stained brains with DAPI to be able to identify structure. We used anatomical structure to verify the locations of our injections in AC and PPC. 


\section{7}

838

839

840

841

842

843

844

845

846

847

848

849

850

\section{1}

852

853

\section{Acknowledgments}

We thank Chengcheng Huang, Ross Williamson, and members of the Runyan lab for comments on the manuscript. This research was supported in part by the University of Pittsburgh Center for Research Computing through the resources provided. Judith Joyce Balcita-Pedicino performed histology and immunohistochemistry. We thank the GENIE project (Janelia) for making GCaMP sensors available for use. We thank the developers of Suite2P and Wavesurfer. This work was supported by the Andrew W. Mellon Predoctoral Fellowship, NIH Predoctoral Training Grant in Basic Neuroscience (T32 NS007433-21), Pew Biomedical Scholars Program, the Searle Scholars Program, the Klingenstein-Simons Fellowship Award in Neuroscience, and NIH grants NIMH DP2MH122404, NINDS R01NS121913.

\section{References}

1. M. R. Cohen, J. H. R. Maunsell, Attention improves performance primarily by reducing interneuronal correlations. Nature Neuroscience 12, 1594-1600 (2009).

2. N. C. Rabinowitz, R. L. Goris, M. Cohen, E. P. Simoncelli, Attention stabilizes the shared gain of V4 populations. eLife 4, e08998 (2015).

3. C. J. McAdams, J. H. Maunsell, Effects of attention on orientation-tuning functions of single neurons in macaque cortical area V4. J Neurosci Official J Soc Neurosci 19, 431-41 (1999).

4. S. Treue, J. H. R. Maunsell, Attentional modulation of visual motion processing in cortical areas MT and MST. Nature 382, 539-541 (1996).

5. J. F. Mitchell, K. A. Sundberg, J. H. Reynolds, Spatial Attention Decorrelates Intrinsic Activity Fluctuations in Macaque Area V4. Neuron 63, 879-888 (2009).

6. M. Vinck, R. Batista-Brito, U. Knoblich, J. A. Cardin, Arousal and Locomotion Make Distinct Contributions to Cortical Activity Patterns and Visual Encoding. Neuron 86, 740-754 (2015).

7. J. F. A. Poulet, C. C. H. Petersen, Internal brain state regulates membrane potential synchrony in barrel cortex of behaving mice. Nature 454, 881-885 (2008).

8. M. Zhou, et al., Scaling down of balanced excitation and inhibition by active behavioral states in auditory cortex. Nature Neuroscience 17, 841-850 (2014).

9. P.-A. Lin, S. K. Asinof, N. J. Edwards, J. S. Isaacson, Arousal regulates frequency tuning in primary auditory cortex. Proc National Acad Sci 116, 25304-25310 (2019).

10. C. M. Niell, M. P. Stryker, Modulation of Visual Responses by Behavioral State in Mouse Visual Cortex. Neuron 65, 472-479 (2010).

11. Y. Fu, et al., A Cortical Circuit for Gain Control by Behavioral State. Cell 156, 1139-1152 (2014).

12. P. J. Mineault, E. Tring, J. T. Trachtenberg, D. L. Ringach, Enhanced Spatial Resolution During Locomotion and Heightened Attention in Mouse Primary Visual Cortex. The Journal of neuroscience : the official journal of the Society for Neuroscience 36, 6382-6392 (2016). 
876 13. P.-O. Polack, J. Friedman, P. Golshani, Cellular mechanisms of brain state-dependent gain

877 modulation in visual cortex. Nat Neurosci 16, 1331-1339 (2013).

878 14. M. J. McGinley, S. V. David, D. A. McCormick, Cortical Membrane Potential Signature of 879 Optimal States for Sensory Signal Detection. Neuron 87, 179-192 (2015).

880 15. C. Bennett, S. Arroyo, S. Hestrin, Subthreshold Mechanisms Underlying State-Dependent 881 Modulation of Visual Responses. Neuron 80, 350-357 (2013).

882 16. D. Shimaoka, K. D. Harris, M. Carandini, Effects of Arousal on Mouse Sensory Cortex 883 Depend on Modality. Cell Reports 25, 3230 (2018).

884 17. D. M. Schneider, A. Nelson, R. Mooney, A synaptic and circuit basis for corollary discharge in 885 the auditory cortex. Nature 513, 189-194 (2014).

886 18. I. Yavorska, M. Wehr, Effects of Locomotion in Auditory Cortex Are Not Mediated by the VIP 887 Network. Front Neural Circuit 15, 618881 (2021).

888 19. R. Tremblay, S. Lee, B. Rudy, GABAergic Interneurons in the Neocortex: From Cellular 889 Properties to Circuits. Neuron 91, 260-292 (2016).

890 20. E. E. Fanselow, K. A. Richardson, B. W. Connors, Selective, state-dependent activation of 891 somatostatin-expressing inhibitory interneurons in mouse neocortex. Journal of neurophysiology $892100,2640-2652$ (2008).

893 21. M. Beierlein, J. R. Gibson, B. W. Connors, A network of electrically coupled interneurons 894 drives synchronized inhibition in neocortex. Nat Neurosci 3, 904-910 (2000).

895 22. N. Chen, H. Sugihara, M. Sur, An acetylcholine-activated microcircuit drives temporal 896 dynamics of cortical activity. Nature Neuroscience 18, 892-902 (2015).

897 23. Z. Xiang, J. R. Huguenard, D. A. Prince, Cholinergic switching within neocortical inhibitory 898 networks. Science 281, 985-988 (1998).

899 24. Y. Kawaguchi, T. Shindou, Noradrenergic Excitation and Inhibition of GABAergic Cell Types 900 in Rat Frontal Cortex. J Neurosci 18, 6963-6976 (1998).

901 25. K. V. Kuchibhotla, et al., Parallel processing by cortical inhibition enables context-dependent 902 behavior. Nature Neuroscience (2016) https:/doi.org/10.1038/nn.4436.

903 26. C. K. Pfeffer, M. Xue, M. He, Z. J. Huang, M. Scanziani, Inhibition of Inhibition in Visual 904 Cortex: The Logic of Connections Between Molecularly Distinct Interneurons. Nat Neurosci 16, 905 1068-1076 (2013).

906 27. M. Dipoppa, et al., Vision and Locomotion Shape the Interactions between Neuron Types in 907 Mouse Visual Cortex. Neuron 98, 602-615.e8 (2018).

908 28. P. Garcia-Junco-Clemente, et al., An inhibitory pull-push circuit in frontal cortex. Nat Neurosci $90920,389-392$ (2017). 
910 29. H.-J. Pi, et al., Cortical interneurons that specialize in disinhibitory control. Nature 503, 521$911524(2013)$.

912 30. M. M. Karnani, et al., Opening Holes in the Blanket of Inhibition: Localized Lateral

913 Disinhibition by VIP Interneurons. J Neurosci 36, 3471-3480 (2016).

914 31. E. Fino, R. Yuste, Dense Inhibitory Connectivity in Neocortex. Neuron 69, 1188-1203 (2011).

915 32. H. Xu, H.-Y. Jeong, R. Tremblay, B. Rudy, Neocortical Somatostatin-Expressing GABAergic 916 Interneurons Disinhibit the Thalamorecipient Layer 4. Neuron 77, 155-167 (2013).

917 33. H. Adesnik, W. Bruns, H. Taniguchi, Z. J. Huang, M. Scanziani, A neural circuit for spatial 918 summation in visual cortex. Nature 490, 226-231 (2012).

919 34. N. R. Wilson, C. A. Runyan, F. L. Wang, M. Sur, Division and subtraction by distinct cortical 920 inhibitory networks in vivo. Nature 488, 343-348 (2012).

921 35. R. V. Rikhye, M. Yildirim, M. Hu, V. Breton-Provencher, M. Sur, Reliable sensory processing 922 in mouse visual cortex through cooperative interactions between somatostatin and parvalbumin 923 interneurons. J Neurosci, JN-RM-3176-20 (2021).

924 36. J. Veit, R. Hakim, M. P. Jadi, T. J. Sejnowski, H. Adesnik, Cortical gamma band

925 synchronization through somatostatin interneurons. Nature Neuroscience 20, 951-959 (2017).

926 37. H. K. Kato, S. N. Gillet, J. S. Isaacson, Flexible Sensory Representations in Auditory Cortex

927 Driven by Behavioral Relevance. Neuron 88, 1027-1039 (2015).

928 38. X.-J. Wang, G. R. Yang, A disinhibitory circuit motif and flexible information routing in the 929 brain. Curr Opin Neurobiol 49, 75-83 (2018).

930 39. Y. Kim, et al., Brain-wide Maps Reveal Stereotyped Cell-Type-Based Cortical Architecture 931 and Subcortical Sexual Dimorphism. Cell 171, 456-469.e22 (2017).

932 40. S. J. Dienel, et al., Distinct Laminar and Cellular Patterns of GABA Neuron Transcript

933 Expression in Monkey Prefrontal and Visual Cortices. Cereb Cortex 31, bhaa341- (2020).

934 41. H. Taniguchi, et al., A resource of Cre driver lines for genetic targeting of GABAergic neurons 935 in cerebral cortex. Neuron 71, 995-1013 (2011).

936 42. L. Madisen, et al., A robust and high-throughput Cre reporting and characterization system for 937 the whole mouse brain. Nature Neuroscience 13, 133-140 (2009).

938 43. T.-W. Chen, et al., Ultrasensitive fluorescent proteins for imaging neuronal activity. Nature $939499,295-300(2013)$.

940 44. K. Nakamura, Auditory spatial discriminatory and mnemonic neurons in rat posterior parietal 941 cortex. Journal of neurophysiology 82, 2503-2517 (1999).

942 45. C. A. Runyan, E. Piasini, S. Panzeri, C. D. Harvey, Distinct timescales of population coding 943 across cortex. Nature 548, 92-96 (2017). 
944 46. J. W. Pillow, et al., Spatio-temporal correlations and visual signalling in a complete neuronal

945 population. Nature 454, 995-999 (2008).

946 47. I. M. Park, M. L. R. Meister, A. C. Huk, J. W. Pillow, Encoding and decoding in parietal cortex 947 during sensorimotor decision-making. Nature Neuroscience 17, 1395-1403 (2014).

948 48. M. Minderer, K. D. Brown, C. D. Harvey, The Spatial Structure of Neural Encoding in Mouse 949 Posterior Cortex during Navigation. Neuron (2019) https:/doi.org/10.1016/j.neuron.2019.01.029.

950 49. D. A. Nitz, Tracking route progression in the posterior parietal cortex. Neuron 49, 747-756 951 (2006).

952 50. J. R. Whitlock, G. Pfuhl, N. Dagslott, M.-B. Moser, E. I. Moser, Functional split between 953 parietal and entorhinal cortices in the rat. Neuron 73, 789-802 (2012).

954 51. M. Krumin, J. J. Lee, K. D. Harris, M. Carandini, Decision and navigation in mouse parietal 955 cortex. Elife 7, e42583 (2018).

956 52. C. D. Harvey, P. Coen, D. W. Tank, Choice-specific sequences in parietal cortex during a 957 virtual-navigation decision task. Nature 484, 62-68 (2012).

958 53. A. M. Licata, et al., Posterior Parietal Cortex Guides Visual Decisions in Rats. J Neurosci 37, 959 4954-4966 (2017).

960 54. G. H. Otazu, L.-H. Tai, Y. Yang, A. M. Zador, Engaging in an auditory task suppresses 961 responses in auditory cortex. Nature Neuroscience 12, 646-654 (2009).

962 55. J. Fritz, S. Shamma, M. Elhilali, D. Klein, Rapid task-related plasticity of spectrotemporal 963 receptive fields in primary auditory cortex. Nat Neurosci 6, 1216-1223 (2003).

964 56. D. Saderi, Z. P. Schwartz, C. R. Heller, J. R. Pennington, S. V. David, Dissociation of task 965 engagement and arousal effects in auditory cortex and midbrain. Elife 10, e60153 (2021).

966 57. M. J. Goard, G. N. Pho, J. Woodson, M. Sur, Distinct roles of visual, parietal, and frontal 967 motor cortices in memory-guided sensorimotor decisions. eLife (2016).

968 58. G. N. Pho, M. J. Goard, J. Woodson, B. Crawford, M. Sur, Task-dependent representations of 969 stimulus and choice in mouse parietal cortex. Nat Commun 9, 2596 (2018).

970 59. A. M. Packer, R. Yuste, Dense, unspecific connectivity of neocortical parvalbumin-positive 971 interneurons: a canonical microcircuit for inhibition? The Journal of neuroscience : the official 972 journal of the Society for Neuroscience 31, 13260-13271 (2011).

973 60. S. B. Hofer, et al., Differential connectivity and response dynamics of excitatory and inhibitory 974 neurons in visual cortex. Nature Neuroscience 14, 1045-1052 (2011).

975 61. H. Ko, et al., Functional specificity of local synaptic connections in neocortical networks. $976 \quad$ Nature 473, 87-91 (2011).

977 62. Y. Yoshimura, J. L. M. Dantzker, E. M. Callaway, Excitatory cortical neurons form fine-scale 978 functional networks. Nature 433, 868-873 (2005). 
979 63. Y. Yoshimura, E. M. Callaway, Fine-scale specificity of cortical networks depends on

980 inhibitory cell type and connectivity. Nature Neuroscience 8, 1552-1559 (2005).

981 64. D. V. Buonomano, W. Maass, State-dependent computations: spatiotemporal processing in 982 cortical networks. Nature Reviews Neuroscience 10, 113-125 (2009).

983 65. C. Huang, et al., Circuit Models of Low-Dimensional Shared Variability in Cortical Networks. 984 Neuron 101, 337-348.e4 (2019).

985 66. M. Valente, et al., Correlations enhance the behavioral readout of neural population activity in 986 association cortex. Nat Neurosci 24, 975-986 (2021).

67. M. M. Karnani, et al., Cooperative Subnetworks of Molecularly Similar Interneurons in Mouse Neocortex. Neuron 90, 86-100 (2016).

68. R. A. Andersen, C. A. Buneo, INTENTIONAL MAPS IN POSTERIOR PARIETAL CORTEX.

69. L. Waschke, S. Tune, J. Obleser, Local cortical desynchronization and pupil-linked arousal differentially shape brain states for optimal sensory performance. Elife 8, e51501 (2019).

70. J. Urban-Ciecko, A. L. Barth, Somatostatin-expressing neurons in cortical networks. Nat Rev 994 Neurosci 17, 401-409 (2016).

71. J. M. Pakan, et al., Behavioral-state modulation of inhibition is context-dependent and cell type specific in mouse visual cortex. Elife 5, e14985 (2016).

1005 76. A. G. Khan, et al., Distinct learning-induced changes in stimulus selectivity and interactions of 1006 GABAergic interneuron classes in visual cortex. Nature Neuroscience 21, 851-859 (2018).

1007 77. G. Rothschild, I. Nelken, A. Mizrahi, Functional organization and population dynamics in the 1008 mouse primary auditory cortex. Nat Neurosci 13, 353-360 (2010).

1009 78. R. Rosenbaum, M. A. Smith, A. Kohn, J. E. Rubin, B. Doiron, The spatial structure of 1010 correlated neuronal variability. Nat Neurosci 20, 107-114 (2017).

1011 79. M. A. Smith, A. Kohn, Spatial and Temporal Scales of Neuronal Correlation in Primary Visual 1012 Cortex. The Journal of neuroscience : the official journal of the Society for Neuroscience 28, 1013 12591-12603 (2008). 
1014

1015

1016

1017

1018

1019

1020

1021

1022

1023

1024

1025

1026

1027

1028

1029

1030

1031

1032

1033

1034

1035

1036

1037

1038
80. M. I. Chelaru, V. Dragoi, Negative Correlations in Visual Cortical Networks. Cereb Cortex 26, 246-256 (2016).

81. D. P. A. Schulz, M. Sahani, M. Carandini, Five key factors determining pairwise correlations in visual cortex. J Neurophysiol 114, 1022-1033 (2015).

82. M. A. Smith, M. A. Sommer, Spatial and Temporal Scales of Neuronal Correlation in Visual Area V4. J Neurosci 33, 5422-5432 (2013).

83. S. Safavi, et al., Nonmonotonic spatial structure of interneuronal correlations in prefrontal microcircuits. Proc National Acad Sci 115, 201802356 (2018).

84. H. K. Kato, S. K. Asinof, J. S. Isaacson, Network-Level Control of Frequency Tuning in Auditory Cortex. Neuron 95, 412-423.e4 (2017).

85. A. C. Huk, M. N. Shadlen, Neural activity in macaque parietal cortex reflects temporal integration of visual motion signals during perceptual decision making. The Journal of neuroscience : the official journal of the Society for Neuroscience 25, 10420-10436 (2005).

86. J. K. Fitzgerald, D. J. Freedman, J. A. Assad, Generalized associative representations in parietal cortex. Nature Neuroscience 14, 1075-1079 (2011).

87. M. Elhilali, J. B. Fritz, D. J. Klein, J. Z. Simon, S. A. Shamma, Dynamics of precise spike timing in primary auditory cortex. The Journal of neuroscience : the official journal of the Society for Neuroscience 24, 1159-1172 (2004).

88. M. Pachitariu, et al., Suite2p: beyond 10,000 neurons with standard two-photon microscopy. Biorxiv, 061507 (2017).

89. J. Friedrich, P. Zhou, L. Paninski, Fast online deconvolution of calcium imaging data. Plos Comput Biol 13, e1005423 (2017).

90. J. Friedman, T. Hastie, R. Tibshirani, Regularization Paths for Generalized Linear Models via Coordinate Descent. Journal of Statistical Software 33 (2010). 

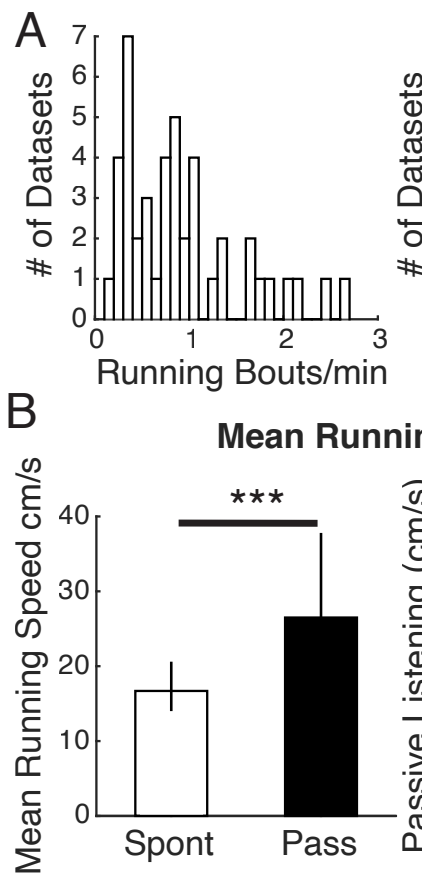

D

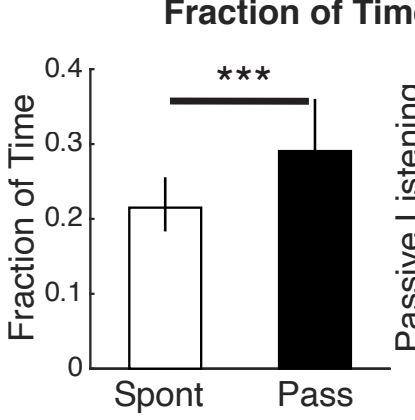

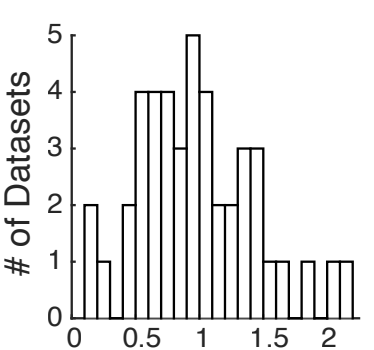

Pupil Dilations/min

Speed

C
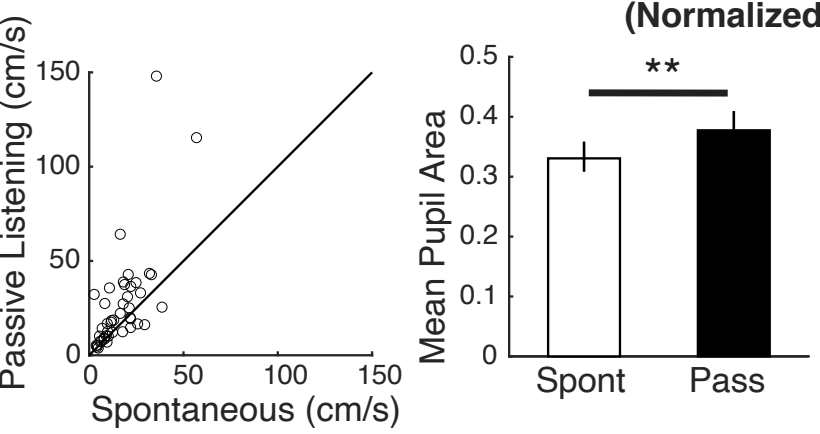

Mean Pupil Area

(Normalized Within Sessions)

$E$

Fraction of Time in High Arousal Cluster
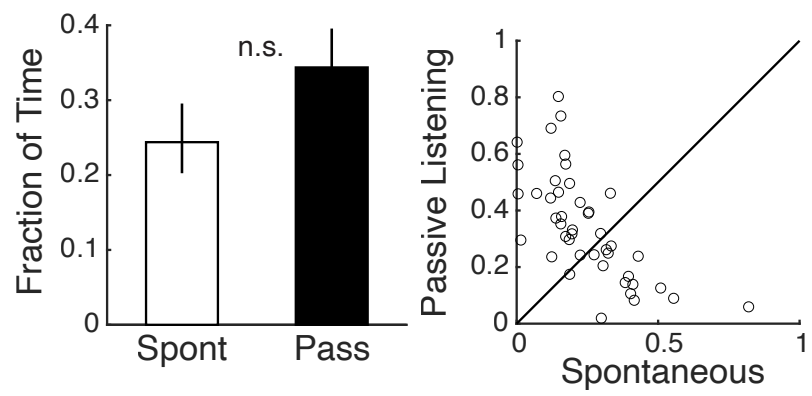

Figure S1: Running behavior and pupil area across imaging sessions. (A) Overall frequency of behavioral state changes, quantified as running bouts (left) and pupil dilations (right), as defined in Materials and Methods; $\mathrm{N}=44$ datasets. (B) Left: Mean running speed in the spontaneous and passive listening behavioral contexts. Right: Mean running speed during the passive listening context plotted against the mean running speed during the spontaneous context, for each imaging session. (C) As in B, for pupil area (normalized across each session). (D) Left: Mean fraction of time spent running in spontaneous and passive listening behavioral contexts. Right: Fraction of time spent running during the passive listening context plotted against the fraction of time spent running during the spontaneous context. $(E)$ As in $D$, but for time spent in the "high arousal cluster", see Materials and Methods. ${ }^{* *} \mathrm{p}<0.001 ;{ }^{* *} \mathrm{p}<.01$; n.s. not significant. Error bars indicate bootstrapped 95\% confidence intervals. See Table S1 for related statistics. 

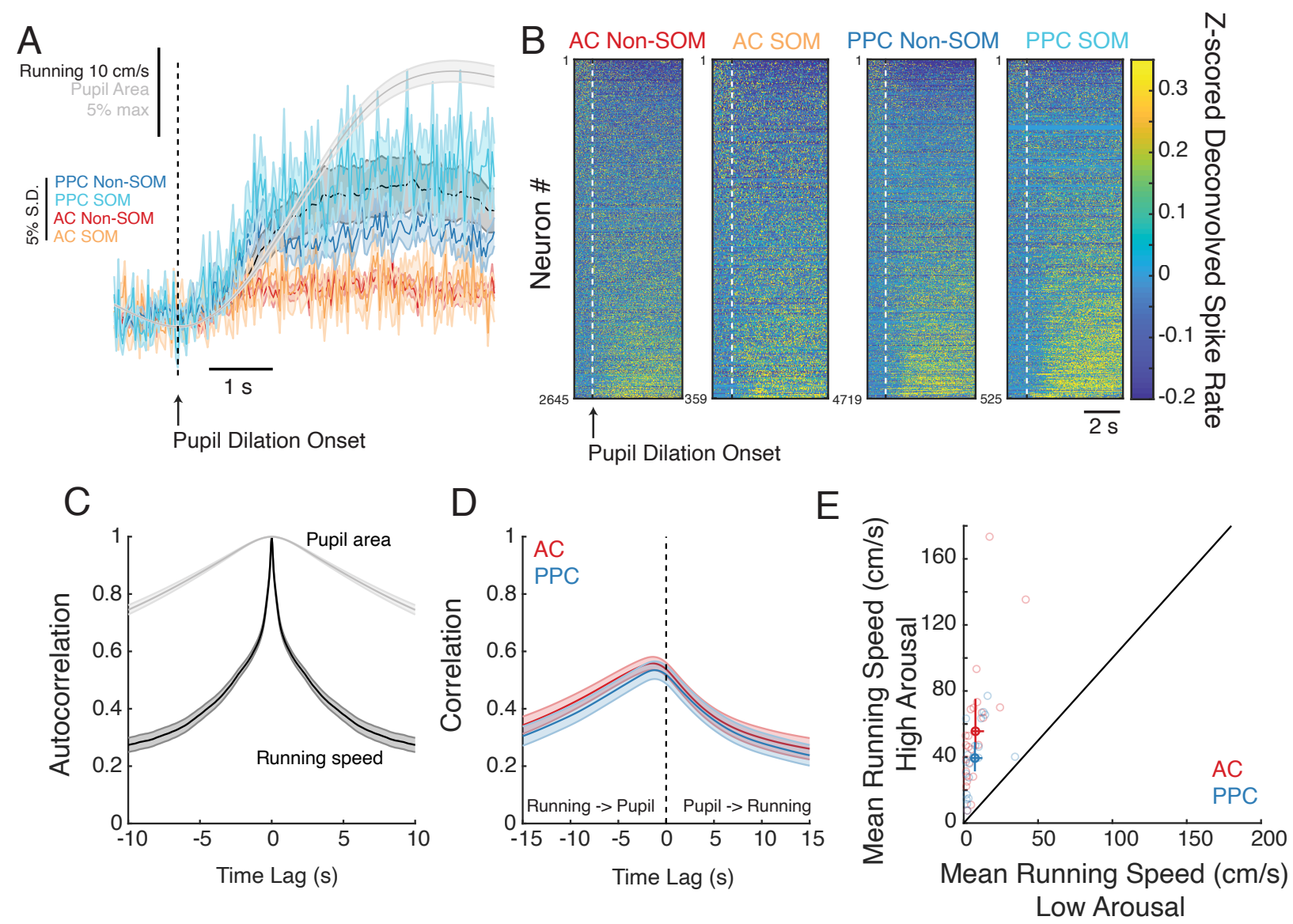

Figure S2: Pupil dilation events, and the relationship between pupil area and running speed. (A) Running speed, pupil area, and single neuron activity, aligned and averaged on pupil dilation events (as compared to running bouts of Figure $3 \mathrm{~A}$ ), $\mathrm{N}=44$ datasets. (B) Average activity, z-scored deconvolved spike rates, of all AC Non-SOM ( $N=2645)$, AC SOM ( $N=359)$, PPC Non-SOM ( $N=4719)$, and PPC SOM ( $N=525)$ neurons, aligned on pupil dilation events. Neurons were sorted by pupil modulation. (C) Autocorrelation of pupil area and running speed, averaged across all datasets $(\mathrm{N}=44)$. (D) Crosscorrelation between running speed and pupil area, averaged across AC datasets (red, $N=24$ ) and PPC datasets (blue, $N=20$ ). (E) Mean running speed in high arousal states (defined as dilated pupil cluster, as in Figure 2) plotted against mean running speed in low arousal states, for individual AC and PPC datasets (light), and averaged across datasets (bold). Shading indicates SEM. 

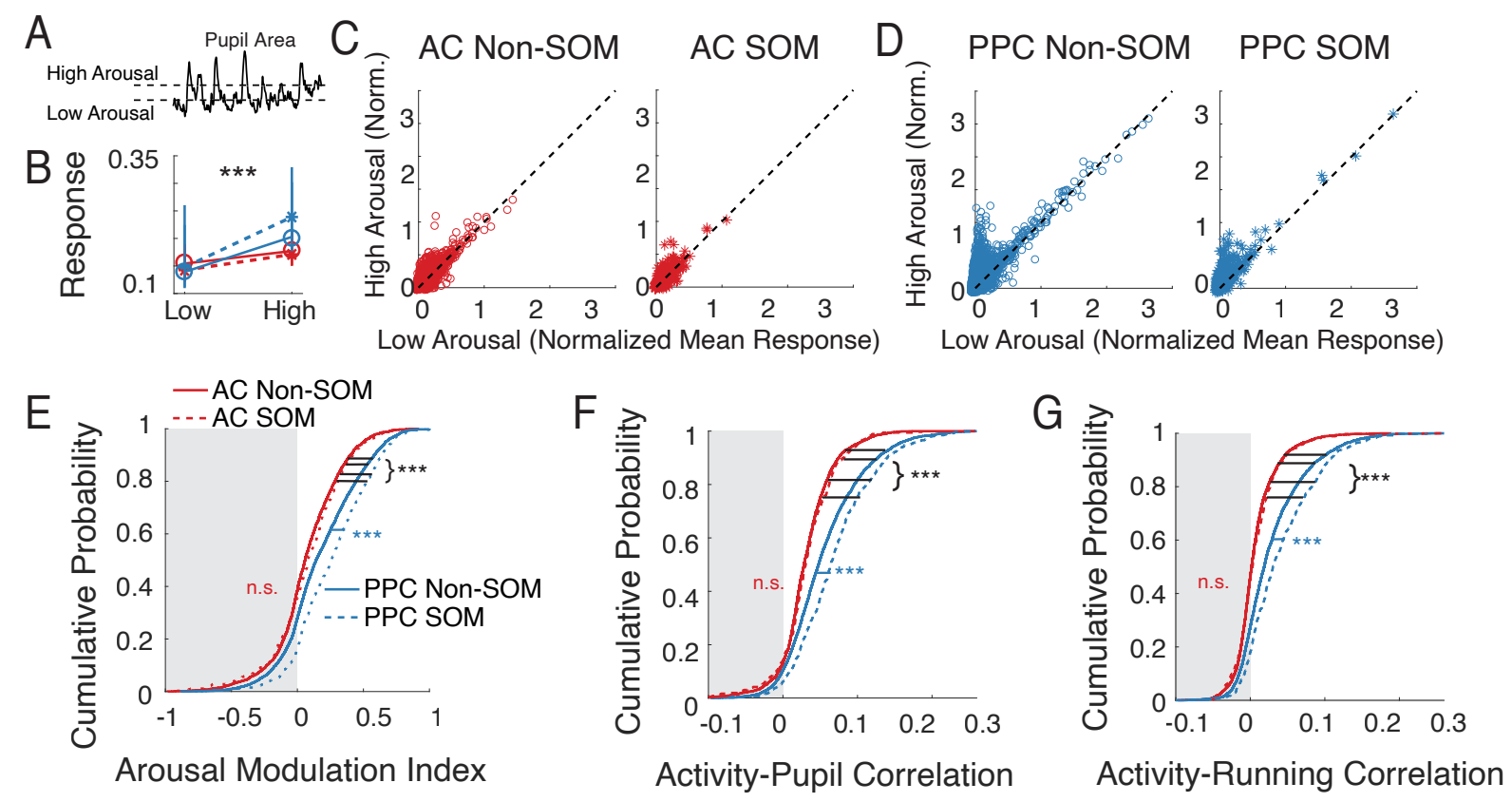

Figure S3: Single cell activity relationships with pupil area and running speed, in the passive listening context. Analyses are the same as presented in Figure $2 \mathrm{C}-\mathrm{I}$, but in the passive listening context. $\mathrm{AC}$ Non-SOM N=2645; AC SOM N=359; PPC Non-SOM N=4719; PPC SOM N=525 for all panels. (A) Example of clustering that sorted pupil area during each imaging frame into low, transition, and high arousal states. (B) Mean responses of AC Non-SOM (red solid), AC SOM (red dotted), PPC Non-SOM (blue solid), and PPC SOM (blue dotted) neurons in low and high arousal states, as defined in $A .{ }^{* * *} p<.001$, lines represent bootstrapped 95\% confidence intervals. (C) Left: Mean activity of AC Non-SOM neurons in high arousal state (classified with clustering as in A), plotted against mean activity in the low arousal state. Right: Mean activity of AC SOM neurons in high arousal vs low arousal states. (D) As in C, for PPC Non-SOM and SOM neurons. (E) Cumulative probability distribution of the arousal modulation index in AC Non-SOM (solid red), AC SOM (dotted red), PPC Non-SOM (solid blue), and PPC SOM (dotted blue) neurons. Arousal modulation index was calculated from the values in C-D, (High-Low)/(High+Low) for each neuron. (F) Cumulative probability distribution of the Pearson correlation between each neuron's activity and pupil area, colors as in E. (G) Cumulative probability distribution of the Pearson correlation between each neuron's activity and running speed. Full summary of statistics an dvalues a vailable in Tables S4-5. 


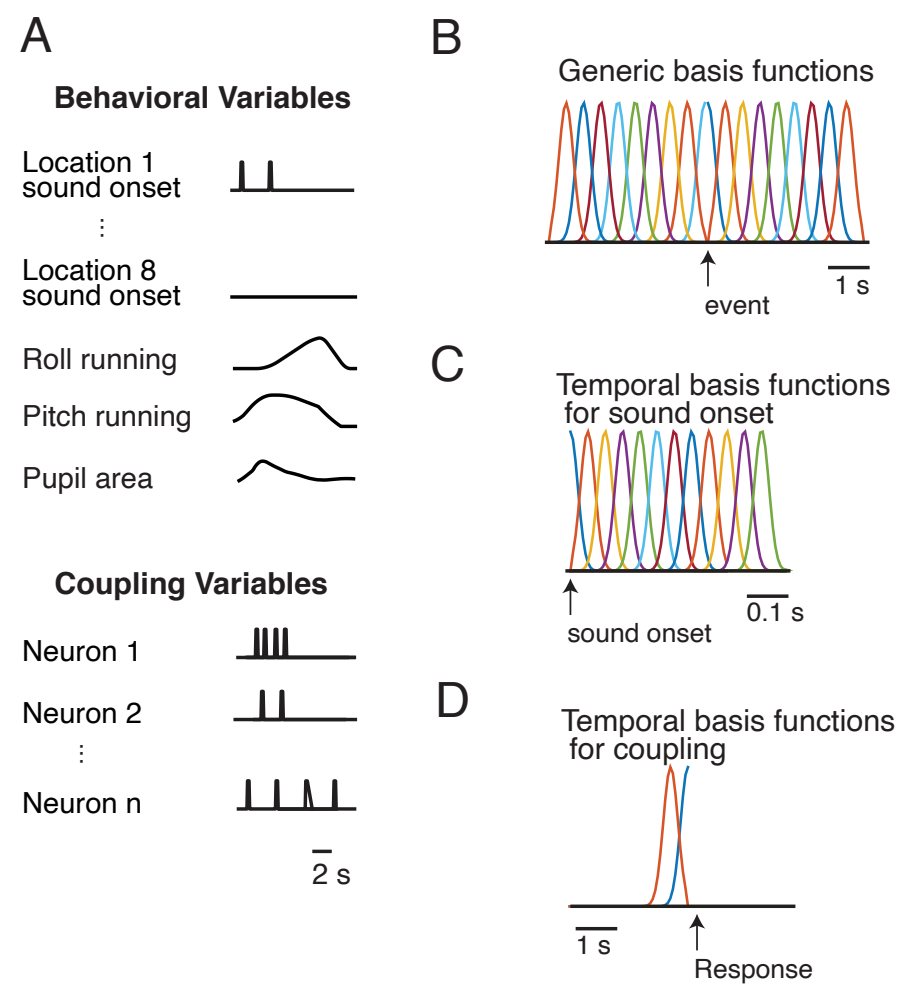

Figure S4: Construction of the predictors used in the encoding models. (A) Behavioral variables and coupling variables used in variants of the Generalized Linear Models (GLMs) to relate neural activity to behavioral variables and/or activity of other simultaneously imaged neurons. Coupling variables could be either Non-SOM neurons or SOM neurons. (B) Depiction of basis functions used to allow for modeling the temporal relationship between behavioral and/or coupling variables to neural activity. The variables in $\mathrm{A}$ were convolved with a set of basis functions moving either forward or backward in time, depending on the type of variable. For example, running velocity in each possible direction was deconvolved with eight basis functions stretching $1 \mathrm{~s}$ in time, prospectively and retrospectively. Thus eight total predictors for each running velocity direction were included in the model. (C) Schematic of the basis functions convolved with sound onset times, for each possible sound location. These spread only forward in time from sound onset. (D) Basis functions convolved with each coupling variable (each simultaneously imaged neuron). 

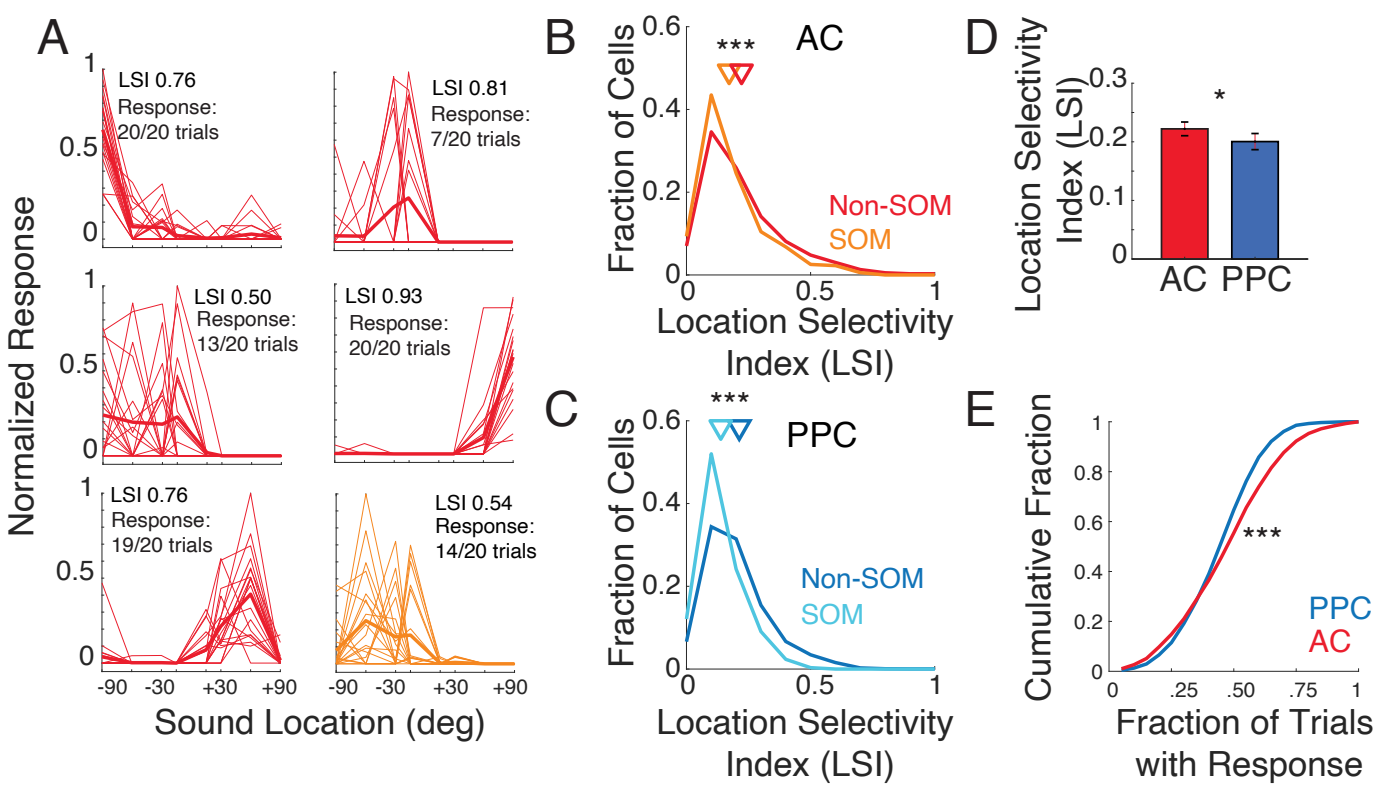

Figure S5: Sound Location Selectivity in AC and PPC. (A) Six example neurons with diverse sound location preferences, ranging from ipsilateral (-90 - 0 degrees) to contralateral locations $(0-+90$ degrees). Thin lines: mean response during sound presentation of individual trials. Thick lines: Trial average. The location selectivity index (LSI) and response reliability are reported for each example cell. Red: Example Non-SOM neurons. Orange: Example SOM neuron. (B) Distribution of Location Selectivity Index (LSI) for Non-SOM ( $\mathrm{N}=2455)$ and SOM $(\mathrm{N}=354)$ neurons in AC. Triangles indicate population means. (C) As in $\mathrm{B}$, for PPC (Non-SOM N=2553; SOM N=298). (D) Mean location selectivity index in AC and PPC. (E) Cumulative distributions of response reliability, the fraction of sound-responsive trials, in AC and PPC. ${ }^{*} p<0.05$, ${ }^{* * *} p<0.001$, error bars indicate SEM. Full summary of values and statistics in Tables S7-8. 


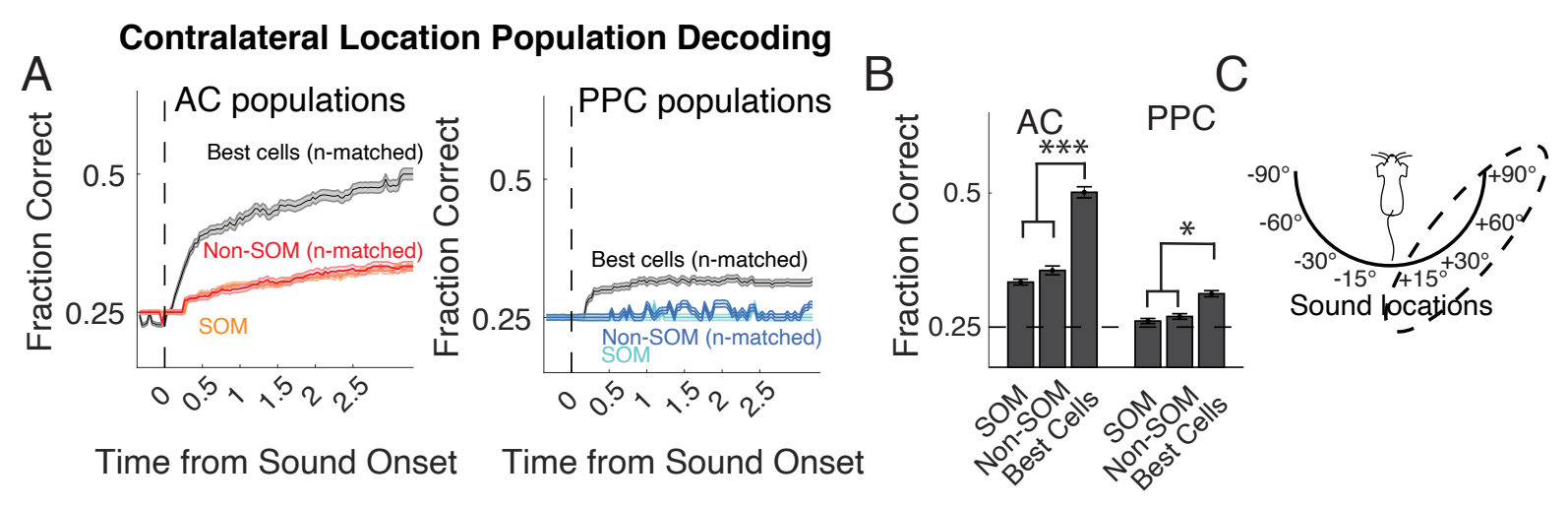

Figure S6: Decoding Sound Location from SOM and Non-SOM Population Activity. (A) As in Figure $4 \mathrm{C}$, but when classifying specific contralateral locations. Chance performance is .25, as there are four possible contralateral locations. (B) As in Figure 4D, but when classifying within contralateral locations. Dotted line indicates chance performance. $(C)$ The discrimination being performed by the decoder, classifying each trial as one of four possible locations on the contralateral side. ${ }^{* * *} p<0.001,{ }^{*} p<0.05$. See Table S9 for full statistics. 


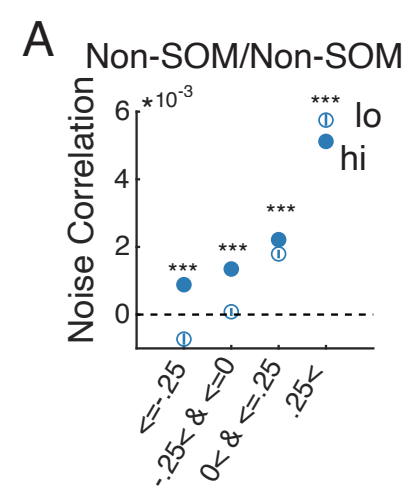

Signal Correlation

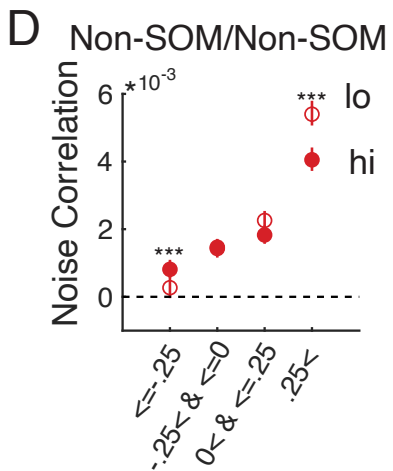

Signal Correlation

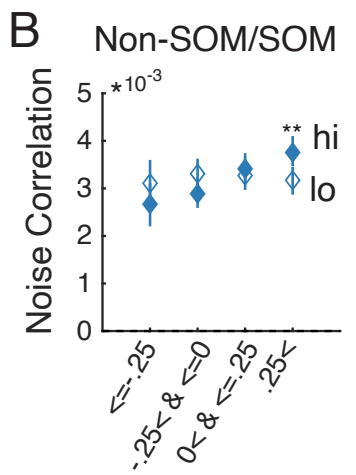

Signal Correlation

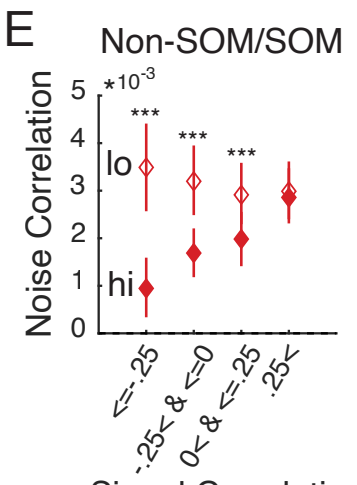

Signal Correlation

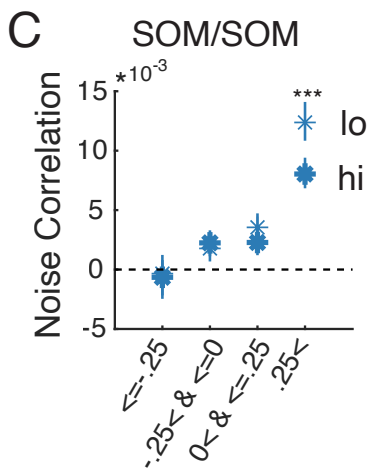

Signal Correlation

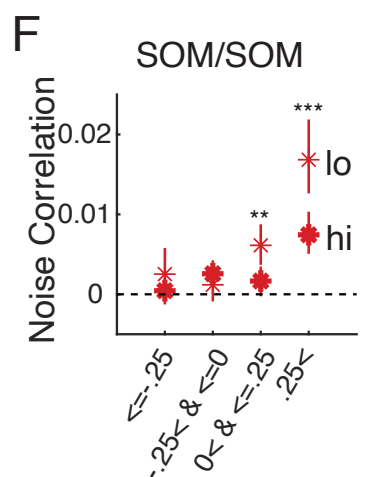

Signal Correlation

Figure S7: Noise correlations tended to be weaker in high arousal states, but the magnitude of the effect depended on signal correlation and cell type. (A) Mean pairwise noise correlation of Non-SOM/Non-SOM neurons in PPC datasets, binned by signal correlation. Filled circles: high arousal, open circles: low arousal. High and low arousal were defined as in Figure 2, by clustering pupil area measurements across the imaging session. Bin 1, N=92989; Bin 2, $N=192997$; $B$ in 3, N=181712; Bin 4, $\mathrm{N}=152621$. (B) As in A, for Non-SOM/SOM pairs in PPC datasets. Filled diamonds: high arousal, open diamonds: low arousal. Bin 1, N= 19046; Bin 2, N=39303; Bin 3, N=37849; Bin 4, N=33121. (C) As in A, for SOM/SOM pairs in PPC datasets. Bolded stars: high arousal, Unbolded stars: low arousal. Bin 1, N=984; Bin 2, N=2412; Bin 3; $N=2277$; Bin 4, $N=1971$. (D-F) As in A-C, for AC datasets. Error bars: bootstrapped $95 \%$ confidence intervals around the mean. ${ }^{* * *} p<0.001,{ }^{* *} p<0.01$, permutation test. Full summary of values and statistics in Table S11. 

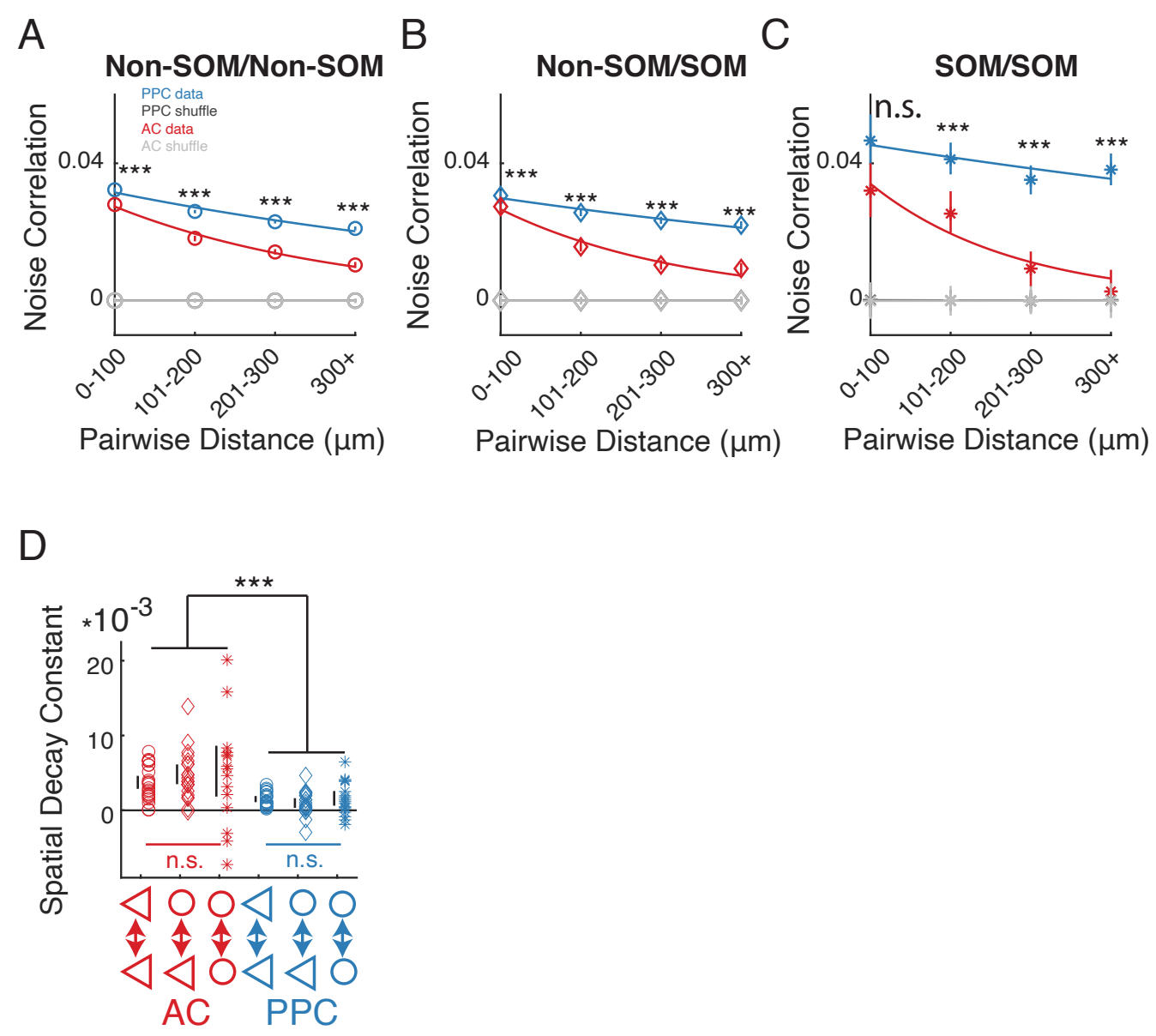

Figure S8: The Spatial Scale of Correlated Variability Differs in AC and PPC, Measured in Running Bout "Trials". (A-D) As in Figure 5D-G, but noise correlations were measured based on responses during running bouts (defined as in Figure 2) as trials in the spontaneous context, rather than on sound stimulus trials in passive listening. Overall trends in noise correlations based on running bouts were similar to those based on sound stimulus responses. Full summary of values and statistics in Tables S14-16. 
Table S1: Comparing Pupil Diameter and Running Speed in AC and PPC Datasets

\begin{tabular}{|c|c|c|c|c|c|}
\hline Group & $\mathbf{N}$ & Mean & $\begin{array}{l}\text { Standard } \\
\text { Deviation }\end{array}$ & $\begin{array}{c}95 \% \\
\text { Confidence } \\
\text { Interval of the } \\
\text { Mean }\end{array}$ & $\begin{array}{c}\text { p value } \\
\text { (unpaired } \\
\text { permutation } \\
\text { test) }\end{array}$ \\
\hline $\begin{array}{l}\mathrm{AC} \text { datasets mean } \\
\mathrm{cm} / \mathrm{s} \text {, passive }\end{array}$ & 24 & 31.5 & 33.4 & $21.8-51.3$ & .20 \\
\hline $\begin{array}{l}\text { PPC datasets, mean } \\
\mathrm{cm} / \mathrm{s} \text {, passive }\end{array}$ & 20 & 20.5 & 15.5 & $15.2-27.8$ & \\
\hline $\begin{array}{l}\text { AC datasets mean } \\
\mathrm{cm} / \mathrm{s} \text {, spontaneous }\end{array}$ & 24 & 17.7 & 12.8 & $13.5-23.6$ & .52 \\
\hline $\begin{array}{l}\text { PPC datasets mean } \\
\mathrm{cm} / \mathrm{s} \text {, spontaneous }\end{array}$ & 20 & 15.4 & 9.1 & $12.2-19.5$ & \\
\hline $\begin{array}{c}\mathrm{AC} \text { datasets mean } \\
\text { pupil diameter, passive }\end{array}$ & 24 & .39 & .12 & $.35-.44$ & .43 \\
\hline $\begin{array}{l}\text { PPC datasets mean } \\
\text { pupil diameter, passive }\end{array}$ & 20 & .36 & .096 & $.32-.41$ & \\
\hline $\begin{array}{l}\text { AC datasets mean } \\
\text { pupil diameter, } \\
\text { spontaneous }\end{array}$ & 24 & .33 & .073 & $.31-.36$ & .73 \\
\hline $\begin{array}{l}\text { PPC datasets mean } \\
\text { pupil diameter, } \\
\text { spontaneous }\end{array}$ & 20 & .33 & .10 & $.29-.38$ & \\
\hline $\begin{array}{l}\text { AC datasets, pupil } \\
\text { dilations } \text { min }^{-1}\end{array}$ & 24 & 1.0 & .52 & $.82-1.2$ & .65 \\
\hline $\begin{array}{l}\text { PPC datasets, pupil } \\
\text { dilations } \mathrm{min}^{-1}\end{array}$ & 20 & .94 & .40 & $.78-1.1$ & \\
\hline $\begin{array}{l}\text { AC datasets, running } \\
\text { bouts } \mathrm{min}^{-1}\end{array}$ & 24 & .87 & .57 & $.66-1.1$ & .59 \\
\hline $\begin{array}{l}\text { PPC datasets, running } \\
\text { bouts } \text { min }^{-1}\end{array}$ & 20 & .97 & .70 & $.72-1.4$ & \\
\hline $\begin{array}{l}\text { AC datasets, fraction } \\
\text { time high cluster } \\
\text { passive }\end{array}$ & 24 & .36 & .20 & $.29-.45$ & .54 \\
\hline $\begin{array}{l}\text { PPC datasets, fraction } \\
\text { time high cluster } \\
\text { passive }\end{array}$ & 20 & .32 & .17 & $.25-.40$ & \\
\hline
\end{tabular}




\begin{tabular}{|c|c|c|c|c|c|}
\hline $\begin{array}{l}\text { AC datasets fraction } \\
\text { time high cluster, } \\
\text { spontaneous }\end{array}$ & 24 & .23 & .15 & $.18-.29$ & .47 \\
\hline $\begin{array}{l}\text { PPC datasets fraction } \\
\text { time high cluster, } \\
\text { spontaneous }\end{array}$ & 20 & .26 & .17 & $.20-.37$ & \\
\hline $\begin{array}{l}\text { AC datasets fraction } \\
\text { time running }>15 \mathrm{~cm} / \mathrm{s} \\
\text { passive }\end{array}$ & 24 & .31 & .20 & $.25-.40$ & .43 \\
\hline $\begin{array}{l}\text { PPC datasets fraction } \\
\text { time running }>15 \mathrm{~cm} / \mathrm{s} \\
\text { passive }\end{array}$ & 20 & .27 & .16 & $.20-.34$ & \\
\hline $\begin{array}{l}\text { AC datasets fraction } \\
\text { time running }>15 \mathrm{~cm} / \mathrm{s} \\
\text { spontaneous }\end{array}$ & 24 & .21 & .13 & $.16-.26$ & .65 \\
\hline $\begin{array}{l}\text { PPC datasets fraction } \\
\text { time running }>15 \mathrm{~cm} / \mathrm{s} \\
\text { spontaneous }\end{array}$ & 20 & .22 & .13 & $.17-.28$ & \\
\hline
\end{tabular}


Table S2: Comparing activity during high and low arousal periods during spontaneous context

\begin{tabular}{|c|c|c|c|c|c|}
\hline Group & $\mathbf{N}$ & Mean & $\begin{array}{l}\text { Standard } \\
\text { Deviation }\end{array}$ & $\begin{array}{c}95 \% \\
\text { Confidence } \\
\text { Interval of the } \\
\text { Mean }\end{array}$ & p value \\
\hline $\begin{array}{c}\text { PPC Non-SOM norm. FR, } \\
\text { high arousal }\end{array}$ & 4719 & .24 & .20 & $.21-.28$ & \\
\hline $\begin{array}{c}\text { PPC Non-SOM norm. FR, } \\
\text { low arousal }\end{array}$ & 4719 & .13 & .11 & $.11-.17$ & \\
\hline Paired permutation & & & & & 0.00 \\
\hline $\begin{array}{l}\text { PPC SOM norm. FR, high } \\
\text { arousal }\end{array}$ & 525 & .30 & .24 & $.25-.34$ & \\
\hline $\begin{array}{c}\text { PPC SOM norm. FR, low } \\
\text { arousal }\end{array}$ & 525 & .14 & .12 & $.11-.17$ & \\
\hline Paired permutation & & & & & 0.00 \\
\hline $\begin{array}{c}\text { AC Non-SOM norm. FR, } \\
\text { high arousal }\end{array}$ & 2645 & .20 & .15 & $.17-.24$ & \\
\hline $\begin{array}{c}\text { AC Non-SOM norm. FR, } \\
\text { low arousal }\end{array}$ & 2645 & .18 & .12 & $.15-.21$ & \\
\hline Paired permutation & & & & & 0.00 \\
\hline $\begin{array}{l}\text { AC SOM norm. FR, high } \\
\text { arousal }\end{array}$ & 359 & .20 & .22 & $.17-.24$ & \\
\hline $\begin{array}{c}\text { AC SOM norm. FR, low } \\
\text { arousal }\end{array}$ & 359 & .17 & .14 & $.15-.22$ & \\
\hline Paired permutation & & & & & .0029 \\
\hline $\begin{array}{l}\text { AC SOM arousal modulation } \\
\text { index }^{1}\end{array}$ & 359 & .057 & .29 & $.027-.085$ & \\
\hline $\begin{array}{l}\text { AC Non-SOM, arousal } \\
\text { modulation index }{ }^{2}\end{array}$ & 2645 & .035 & .28 & $.025-.046$ & \\
\hline $\begin{array}{l}\text { PPC Non-SOM arousal } \\
\text { modulation index }\end{array}$ & 4719 & .25 & .31 & $.24-.26$ & \\
\hline $\begin{array}{l}\text { PPC SOM arousal } \\
\text { modulation index }\end{array}$ & 525 & .35 & .31 & $.32-.37$ & \\
\hline
\end{tabular}


bioRxiv preprint doi: https://doi.org/10.1101/2022.01.18.476770; this version posted January 20, 2022. The copyright holder for this preprint (which was not certified by peer review) is the author/funder, who has granted bioRxiv a license to display the preprint in perpetuity. It is made available under aCC-BY-NC-ND 4.0 International license.

Kruskal Wallis ${ }^{1-4}$

7.2E-12

Unpaired permutation ${ }^{1,2}$

Unpaired permutation, ${ }^{1,3}$

Unpaired permutation, $, 2,3$

Unpaired permutation, ${ }^{1,4}$

Unpaired permutation, ${ }^{2,4}$

Unpaired permutation, ${ }^{3,4}$ 
Table S3: Correlation coefficients with pupil diameter and running speed during spontaneous context

\begin{tabular}{|c|c|c|c|c|c|}
\hline Group & $\mathbf{N}$ & Mean & $\begin{array}{l}\text { Standard } \\
\text { Deviation }\end{array}$ & $\begin{array}{c}\quad 95 \% \\
\text { Confidence } \\
\text { Interval of } \\
\text { the Mean }\end{array}$ & p value \\
\hline $\begin{array}{l}\text { AC Non-SOM, pupil } \\
\text { correlation coefficient }{ }^{1}\end{array}$ & 2645 & .0057 & .033 & $.0045-.0070$ & \\
\hline $\begin{array}{l}\text { AC SOM pupil correlation } \\
\text { coefficient }^{2}\end{array}$ & 359 & .0091 & .031 & $.0061-.12$ & \\
\hline $\begin{array}{l}\text { PPC Non-SOM pupil } \\
\text { correlation coefficient }^{3}\end{array}$ & 4719 & .035 & .043 & $.034-.036$ & \\
\hline $\begin{array}{l}\text { PPC SOM pupil correlation } \\
\text { coefficient }^{4}\end{array}$ & 525 & .055 & .049 & $.051-.059$ & \\
\hline Kruskal Wallis ${ }^{1-4}$ & & & & & $6.3 \mathrm{E}-250$ \\
\hline Unpaired permutation ${ }^{1,2}$ & & & & & .063 \\
\hline Unpaired permutation ${ }^{1,3}$ & & & & & $<.00010$ \\
\hline Unpaired permutation ${ }^{, 2,3}$ & & & & & $<.00010$ \\
\hline Unpaired permutation ${ }^{, 1,4}$ & & & & & $<.00010$ \\
\hline Unpaired permutation ${ }^{, 2,4}$ & & & & & $<.00010$ \\
\hline Unpaired permutation 3,4 & & & & & $<.00010$ \\
\hline $\begin{array}{l}\text { AC Non-SOM, running } \\
\text { speed correlation coefficient }{ }^{1}\end{array}$ & 2645 & .0020 & .034 & $.00060-.0033$ & \\
\hline $\begin{array}{l}\text { AC SOM running speed } \\
\text { correlation coefficient }^{2}\end{array}$ & 359 & .0048 & .034 & $.0013-.0089$ & \\
\hline $\begin{array}{c}\text { PPC Non-SOM running } \\
\text { speed correlation coefficient }{ }^{3}\end{array}$ & 4719 & .033 & .044 & $.032-.034$ & \\
\hline $\begin{array}{l}\text { PPC SOM running speed } \\
\text { correlation coefficient }^{4}\end{array}$ & 525 & .057 & .055 & $.053-.062$ & \\
\hline Kruskal Wallis ${ }^{1-4}$ & & & & & 0.00 \\
\hline Unpaired permutation ${ }^{1,2}$ & & & & & .15 \\
\hline Unpaired permutation ${ }^{, 1,3}$ & & & & & $<.00010$ \\
\hline Unpaired permutation ${ }^{, 2,3}$ & & & & & $<.00010$ \\
\hline Unpaired permutation ${ }^{1,4}$ & & & & & $<.00010$ \\
\hline Unpaired permutation ${ }^{, 2,4}$ & & & & & $<.00010$ \\
\hline Unpaired permutation ${ }^{, 3,4}$ & & & & & $<.00010$ \\
\hline
\end{tabular}


Table S4: Comparing activity during high and low arousal periods during passive listening context

Group N Mean $\begin{gathered}\text { Standard } \\ \text { Deviation }\end{gathered}$

PPC Non-SOM norm. FR, high arousal

PPC Non-SOM norm. FR, low arousal

Paired permutation

PPC SOM norm. FR, high arousal

PPC SOM norm. FR, low arousal

Paired permutation

AC Non-SOM norm. FR, high arousal

AC Non-SOM norm. FR, low arousal

Paired permutation

4719

.13

.12
4719 .20

.14

.20

.17

0.00

525

525

.20

.15

$\mathbf{9 5 \%}$
Confidence
Interval of the
Mean
$.18-.33$
$.11-.26$

$.20-.30$

$.11-.22$ p value

0.00

$.15-.22$

$.13-.19$

0.00

359

.17

.13

$.15-.22$

359

.14

.12

$.12-.18$

Paired permutation

AC Non-SOM, arousal modulation index ${ }^{1}$

2645

.072

.26

$.063-.083$

AC SOM arousal modulation

359

.079

.28

$.049-.11$

PPC Non-SOM arousal

4719

.18

.29

$.17-.19$

modulation index ${ }^{3}$

PPC SOM arousal

525

.26

.28

$.24-.29$

Kruskal Wallis ${ }^{1-4}$ Unpaired permutation ${ }^{1,2}$

Unpaired permutation ${ }^{1,3}$

Unpaired permutation, ${ }^{2,3}$
2.1E-66

$<.00010$

$<.00010$ 
bioRxiv preprint doi: https://doi.org/10.1101/2022.01.18.476770; this version posted January 20, 2022. The copyright holder for this preprint (which was not certified by peer review) is the author/funder, who has granted bioRxiv a license to display the preprint in perpetuity. It is made available under aCC-BY-NC-ND 4.0 International license.

Unpaired permutation, ${ }^{1,4}$

$<.00010$

Unpaired permutation, ${ }^{2,4}$

$<.00010$

Unpaired permutation, ${ }^{3,4}$

$<.00010$ 
Table S5: Correlation coefficients with pupil diameter and running speed during passive listening context

\begin{tabular}{|c|c|c|c|c|c|}
\hline Group & $\mathbf{N}$ & Mean & $\begin{array}{l}\text { Standard } \\
\text { Deviation }\end{array}$ & $\begin{array}{c}\quad 95 \% \\
\text { Confidence } \\
\text { Interval of } \\
\text { the Mean }\end{array}$ & p value \\
\hline $\begin{array}{l}\text { AC Non-SOM, pupil } \\
\text { correlation coefficient }^{1}\end{array}$ & 2645 & .0096 & .031 & $.0081-.010$ & \\
\hline $\begin{array}{l}\text { AC SOM pupil correlation } \\
\text { coefficient }^{2}\end{array}$ & 359 & .010 & .033 & $.0074-.014$ & \\
\hline $\begin{array}{l}\text { PPC Non-SOM pupil } \\
\text { correlation coefficient }^{3}\end{array}$ & 4719 & .030 & .042 & $.029-.031$ & \\
\hline $\begin{array}{l}\text { PPC SOM pupil correlation } \\
\text { coefficient }^{4}\end{array}$ & 525 & .043 & .044 & $.038-.046$ & \\
\hline Kruskal Wallis ${ }^{1-4}$ & & & & & 5.3E-115 \\
\hline Unpaired permutation ${ }^{1,2}$ & & & & & .63 \\
\hline Unpaired permutation ${ }^{1,3}$ & & & & & $<.00010$ \\
\hline Unpaired permutation ${ }^{, 2,3}$ & & & & & $<.00010$ \\
\hline Unpaired permutation ${ }^{, 1,4}$ & & & & & $<.00010$ \\
\hline Unpaired permutation ${ }^{, 2,4}$ & & & & & $<.00010$ \\
\hline Unpaired permutation 3,4 & & & & & $<.00010$ \\
\hline $\begin{array}{l}\text { AC Non-SOM, running } \\
\text { speed correlation coefficient }\end{array}$ & 2645 & .0067 & .029 & $.0056-.0078$ & \\
\hline $\begin{array}{l}\text { AC SOM running speed } \\
\text { correlation coefficient }^{2}\end{array}$ & 359 & .0076 & .30 & $.0050-.011$ & \\
\hline $\begin{array}{c}\text { PPC Non-SOM running } \\
\text { speed correlation coefficient }{ }^{3}\end{array}$ & 4719 & .029 & .042 & $.028-.030$ & \\
\hline $\begin{array}{l}\text { PPC SOM running speed } \\
\text { correlation coefficient }^{4}\end{array}$ & 525 & .045 & .047 & $.038-.046$ & \\
\hline Kruskal Wallis $^{1-4}$ & & & & & $1.2 \mathrm{E}-146$ \\
\hline Unpaired permutation ${ }^{1,2}$ & & & & & .59 \\
\hline Unpaired permutation ${ }^{, 1,3}$ & & & & & $<.00010$ \\
\hline Unpaired permutation ${ }^{, 2,3}$ & & & & & $<.00010$ \\
\hline Unpaired permutation ${ }^{1,4}$ & & & & & $<.00010$ \\
\hline Unpaired permutation ${ }^{, 2,4}$ & & & & & $<.00010$ \\
\hline Unpaired permutation ${ }^{, 3,4}$ & & & & & $<.00010$ \\
\hline
\end{tabular}


Table S6: Pupil and Running Contributions, from Encoding Model in Figure 3

\begin{tabular}{|c|c|c|c|c|c|}
\hline Group & $\mathbf{n}$ & Mean & $\begin{array}{l}\text { Standard } \\
\text { Deviation }\end{array}$ & $\begin{array}{c}95 \% \\
\text { Confidence } \\
\text { Interval of } \\
\text { the Mean }\end{array}$ & p value \\
\hline $\begin{array}{l}\text { AC Non-SOM, pupil } \\
\text { diameter contribution }^{1}\end{array}$ & 2476 & 0.0027 & 0.0086 & $0.0023-0.0031$ & \\
\hline $\begin{array}{l}\text { AC SOM, pupil diameter } \\
\text { contribution }^{2}\end{array}$ & 330 & 0.0036 & 0.0123 & $0.0025-0.0049$ & \\
\hline $\begin{array}{l}\text { PPC Non-SOM, pupil } \\
\text { diameter contribution }^{3}\end{array}$ & 4525 & 0.0029 & 0.0060 & $0.0028-0.0031$ & \\
\hline $\begin{array}{l}\text { PPC SOM, pupil diameter } \\
\text { contribution }^{4}\end{array}$ & 505 & 0.0034 & 0.0059 & 0.0029-.0040 & \\
\hline Kruskal Wallis ${ }^{1-4}$ & & & & & $<0.0001$ \\
\hline Unpaired permutation ${ }^{1,2}$ & & & & & 0.0780 \\
\hline Unpaired permutation ${ }^{, 1,3}$ & & & & & 0.1259 \\
\hline Unpaired permutation ${ }^{2,3}$ & & & & & 0.2360 \\
\hline Unpaired permutation ${ }^{1,4}$ & & & & & 0.0619 \\
\hline Unpaired permutation ${ }^{, 2,4}$ & & & & & 0.9520 \\
\hline Unpaired permutation, ${ }^{3,4}$ & & & & & 0.1070 \\
\hline $\begin{array}{l}\text { AC Non-SOM, running } \\
\text { speed correlation coefficient }{ }^{1}\end{array}$ & 2476 & 0.0020 & 0.0050 & $0.0019-0.0023$ & \\
\hline $\begin{array}{l}\text { AC SOM running speed } \\
\text { correlation coefficient }^{2}\end{array}$ & 330 & 0.0023 & 0.0005 & $0.0019-0.0320$ & \\
\hline $\begin{array}{c}\text { PPC Non-SOM running } \\
\text { speed correlation coefficient }{ }^{3}\end{array}$ & 4525 & 0.0079 & 0.0156 & $0.0075-0.0083$ & \\
\hline $\begin{array}{l}\text { PPC SOM running speed } \\
\text { correlation coefficient }\end{array}$ & 505 & 0.0113 & 0.0181 & $0.0099-0.0131$ & \\
\hline Kruskal Wallis ${ }^{1-4}$ & & & & & $<0.0001$ \\
\hline Unpaired permutation ${ }^{1,2}$ & & & & & 0.4409 \\
\hline Unpaired permutation ${ }^{, 1,3}$ & & & & & 0.0001 \\
\hline Unpaired permutation ${ }^{2,3}$ & & & & & 0.0001 \\
\hline Unpaired permutation ${ }^{1,4}$ & & & & & 0.0001 \\
\hline Unpaired permutation ${ }^{2,4}$ & & & & & 0.0001 \\
\hline Unpaired permutation, 3,4 & & & & & 0.0002 \\
\hline
\end{tabular}




\section{Table S7: Location Selectivity Index}
Group
$\mathbf{N}$
Mean

PPC All Neurons ${ }^{2} \quad 2851$
AC Non-SOM ${ }^{3}$
$\mathrm{AC} \mathrm{SOM}^{4}$

2455

354

PPC Non-SOM ${ }^{5}$

$\mathrm{PPC} \mathrm{SOM}^{6}$

2553

298

Kruskal Wallis ${ }^{3-6}$

Unpaired

permutation $^{3,4}$

Unpaired

permutation $^{3,5}$

Unpaired

permutation $^{3,6}$

Unpaired

permutation $^{4,5}$

Unpaired

permutation $^{4,6}$

Unpaired

permutation $^{5,6}$
0.20

0.19

\section{Standard \\ Deviation}

0.20

0.16

0.20

0.13

0.09

0.16

0.12

0.16

0.13

0.12

0.09
95\%

Confidence

Interval of the

Mean

$0.19-0.21$

$0.18-0.19$

$0.20-0.21$

$0.15-0.18$

$0.19-0.20$

$0.12-0.14$ p value

$<0.01$

$<0.001$

$<0.001$

$<0.0001$

$<0.001$

$<0.05$

$<0.0001$

$<0.0001$

$<0.0001$

$<0.0001$ 
Table S8: Sound Response Reliability

\section{N Mean}

AC All Neurons ${ }^{1}$

PPC All Neurons ${ }^{2}$

2809

2851

AC Non-SOM ${ }^{3}$

2455

$\mathrm{AC} \mathrm{SOM}^{4}$

354

PPC Non-SOM ${ }^{5}$

2553

PPC SOM 6

298

Kruskal Wallis ${ }^{3-6}$

Unpaired

permutation $^{3,4}$

Unpaired

permutation $^{3,5}$

Unpaired

permutation $^{3,6}$

Unpaired

permutation $^{4,5}$

Unpaired

permutation $^{4,6}$

Unpaired

permutation $^{5,6}$
0.49

0.46

Standard

Deviation

0.49

0.50

0.46

0.48

0.20

0.15

0.20

0.19

0.15
0.14

0.15
0.14
95\%

Confidence

Interval of the

Mean

$0.49-0.50$

$0.46-0.47$

$0.49-0.50$

$0.48-0.51$

$0.45-0.47$

$0.46-0.49$ p value

$<0.0001$

$<0.0001$

0.808

$<0.0001$

0.149

$<0.0001$

0.132

0.143 
Table S9: Sound Location Decoding

\begin{tabular}{|c|c|c|c|c|c|}
\hline Group & $\mathbf{n}$ & Mean & $\begin{array}{l}\text { Standard } \\
\text { Deviation }\end{array}$ & $\begin{array}{c}95 \% \\
\text { Confidence } \\
\text { Interval of the } \\
\text { Mean }\end{array}$ & p value \\
\hline AC Best Cells - LR ${ }^{1}$ & $24 \times 10$ & 0.86 & 0.11 & $0.85-0.87$ & \\
\hline AC Non-SOM - LR ${ }^{2}$ & $24 \times 10$ & 0.68 & 0.13 & $0.66-0.69$ & \\
\hline $\mathrm{AC} \mathrm{SOM}-\mathrm{LR}^{3}$ & $24 \times 10$ & 0.68 & 0.12 & $0.66-0.69$ & \\
\hline PPC Best Cells - LR ${ }^{4}$ & $20 \times 10$ & 0.65 & 0.10 & $0.64-0.67$ & \\
\hline PPC Non-SOM - LR ${ }^{5}$ & $20 \times 10$ & 0.53 & 0.09 & $0.52-0.53$ & \\
\hline PPC SOM - LR 6 & $20 \times 10$ & 0.52 & 0.09 & $0.51-0.53$ & \\
\hline Kruskal Wallis ${ }^{1-6}$ & & & & & 0.0001 \\
\hline Unpaired permutation ${ }^{1,2}$ & & & & & 0.0001 \\
\hline Unpaired permutation ${ }^{1,3}$ & & & & & 0.0001 \\
\hline Unpaired permutation ${ }^{1,4}$ & & & & & 0.0001 \\
\hline Unpaired permutation ${ }^{1,5}$ & & & & & 0.0001 \\
\hline Unpaired permutation ${ }^{1,6}$ & & & & & 0.0001 \\
\hline Unpaired permutation ${ }^{2,3}$ & & & & & 0.8167 \\
\hline Unpaired permutation ${ }^{2,4}$ & & & & & 0.1189 \\
\hline Unpaired permutation ${ }^{2,5}$ & & & & & 0.0001 \\
\hline Unpaired permutation ${ }^{2,6}$ & & & & & 0.0001 \\
\hline Unpaired permutation ${ }^{3,4}$ & & & & & 0.1160 \\
\hline Unpaired permutation ${ }^{3,5}$ & & & & & 0.0001 \\
\hline Unpaired permutation 3,6 & & & & & 0.0001 \\
\hline Unpaired permutation $^{4,5}$ & & & & & 0.0001 \\
\hline Unpaired permutation ${ }^{4,6}$ & & & & & 0.0001 \\
\hline Unpaired permutation 5,6 & & & & & 0.4644 \\
\hline $\begin{array}{l}\text { AC High Arousal - LR } \\
\text { (All cells) }\end{array}$ & $24 \times 10$ & 0.83 & 0.16 & $0.81-0.85$ & 0.0003 \\
\hline $\begin{array}{l}\text { AC Low Arousal - LR } \\
\text { (All cells) }\end{array}$ & $24 \times 10$ & 0.78 & 0.12 & $0.76-0.80$ & \\
\hline $\begin{array}{l}\text { PPC High Arousal - LR } \\
\text { (All cells) }\end{array}$ & $20 \times 10$ & 0.54 & 0.13 & $0.53-0.55$ & 0.0600 \\
\hline $\begin{array}{l}\text { PPC Low Arousal - LR } \\
\text { (All cells) }\end{array}$ & $20 \times 10$ & 0.57 & 0.13 & $0.56-0.59$ & \\
\hline
\end{tabular}




\begin{tabular}{|c|c|c|c|c|}
\hline AC Best Cells - Contra $^{1}$ & $24 \times 10$ & 0.44 & 0.13 & $0.45-0.48$ \\
\hline AC Non-SOM - Contra ${ }^{2}$ & $24 \times 10$ & 0.32 & 0.10 & $0.33-0.35$ \\
\hline AC SOM - Contra ${ }^{3}$ & $24 \times 10$ & 0.31 & 0.09 & $0.32-0.34$ \\
\hline PPC Best Cells - Contra ${ }^{4}$ & $20 \times 10$ & 0.28 & 0.09 & $0.26-0.30$ \\
\hline PPC Non-SOM - Contra & $20 \times 10$ & 0.26 & 0.08 & $0.25-0.27$ \\
\hline PPC SOM - Contra ${ }^{6}$ & $20 \times 10$ & 0.25 & 0.09 & $0.25-0.27$ \\
\hline
\end{tabular}

Kruskal Wallis ${ }^{1-6}$

Unpaired permutation ${ }^{1,2}$

$<0.0001$

Unpaired permutation ${ }^{1,3}$

$<0.0001$

Unpaired permutation ${ }^{1,4}$

$<0.0001$

Unpaired permutation ${ }^{1,5}$

$<0.0001$

Unpaired permutation ${ }^{1,6}$

$<0.0001$

Unpaired permutation ${ }^{2,3}$

0.5082

Unpaired permutation ${ }^{2,4}$

0.9354

Unpaired permutation ${ }^{2,5}$

$<0.0001$

Unpaired permutation ${ }^{2,6}$

$<0.0001$

Unpaired permutation ${ }^{3,4}$

0.116

Unpaired permutation ${ }^{3,5}$

$<0.0001$

Unpaired permutation ${ }^{3,6}$

$<0.0001$

Unpaired permutation ${ }^{4,5}$

0.0121

Unpaired permutation ${ }^{4,6}$

0.0118

Unpaired permutation ${ }^{5,6}$

0.9354 
Table S10: Pairwise Noise Correlations (Sound Location Trials)

Group

AC all neurons ${ }^{1}$

PPC all neurons ${ }^{2}$
$\mathbf{N}$

204502

757282

Unpaired permutation ${ }^{1,2}$
Mean

.026

.035

.054

.070

160241

.026

.053

.055

.067

.026

2788

.033

Kruskal Wallis ${ }^{3-5}$

Unpaired permutation ${ }^{3,4}$

Unpaired permutation ${ }^{4,5}$

Unpaired permutation ${ }^{3,5}$

PPC Non-SOM/Non$\mathrm{SOM}^{6}$

PPC Non-SOM/SOM ${ }^{7}$ $\mathrm{PPC}$ SOM/SOM ${ }^{8}$

129319

7644

.040

.071

.033

.068

.073

.10

$<.00010$

$.025-.026$

.025-.026

$.030-.035$

Kruskal Wallis ${ }^{6-8}$

Unpaired permutation ${ }^{6,7}$

$\begin{array}{cc}620319 & .033 \\ 129319 & .040 \\ 7644 & .071\end{array}$

$.033-.033$

Unpaired permutation ${ }^{7,8}$

Unpaired permutation ${ }^{6,8}$

Kruskal Wallis ${ }^{1-8}$

.040-.041

$.069-.073$

$$
\begin{gathered}
.00021 \\
.83 \\
<.00010 \\
<.00010
\end{gathered}
$$

0.00

$<.00010$

$<.00010$

$<.00010$

0.00 
Table S11: High Noise Correlation - Low Noise Correlation

\begin{tabular}{|c|c|c|c|c|c|}
\hline Group & $\mathbf{N}$ & Mean & $\begin{array}{l}\text { Standard } \\
\text { Deviation }\end{array}$ & $\begin{array}{l}95 \% \text { Confidence } \\
\text { Interval of the } \\
\text { Mean }\end{array}$ & $\begin{array}{c}\text { p value } \\
\text { (paired } \\
\text { permutation } \\
\text { test, } \alpha=.0125 \text { ) }\end{array}$ \\
\hline $\begin{array}{c}\text { AC Non-SOM/Non- } \\
\text { SOM bin } 1, \text { high } \\
\text { arousal }\end{array}$ & 11349 & .00081 & .014 & $.00054-.0011$ & .0056 \\
\hline $\begin{array}{l}\text { AC Non-SOM/Non- } \\
\text { SOM bin } 1, \text { low } \\
\text { arousal }\end{array}$ & 11349 & .00027 & .017 & $-.000039-.00058$ & \\
\hline $\begin{array}{l}\text { AC Non-SOM/Non- } \\
\text { SOM bin 2, high } \\
\text { arousal }\end{array}$ & 16401 & .0015 & .016 & $.0012-.0017$ & .82 \\
\hline $\begin{array}{l}\text { AC Non-SOM/Non- } \\
\text { SOM bin 2, low } \\
\text { arousal }\end{array}$ & 16401 & .0014 & .017 & $.0012-.0017$ & \\
\hline $\begin{array}{l}\text { AC Non-SOM/Non- } \\
\text { SOM bin 3, high } \\
\text { arousal }\end{array}$ & 16039 & .0018 & .018 & $.0016-.0021$ & .022 \\
\hline $\begin{array}{l}\text { AC Non-SOM/Non- } \\
\text { SOM bin 3, low } \\
\text { arousal }\end{array}$ & 16039 & .0023 & .018 & $.0020-.0025$ & \\
\hline $\begin{array}{l}\text { AC Non-SOM/Non- } \\
\text { SOM bin 4, high } \\
\text { arousal }\end{array}$ & 18326 & .0041 & .024 & $.0037-.0044$ & 0.00 \\
\hline $\begin{array}{l}\text { AC Non-SOM/Non- } \\
\text { SOM bin 4, low } \\
\text { arousal }\end{array}$ & 18326 & .0054 & .025 & $.0051-.0058$ & \\
\hline $\begin{array}{c}\text { AC Non-SOM/SOM } \\
\text { bin } 1 \text {, high arousal }\end{array}$ & 3190 & .00094 & .018 & $.00034-.0016$ & 0.00 \\
\hline $\begin{array}{c}\text { AC Non-SOM/SOM } \\
\text { bin } 1 \text {, low arousal }\end{array}$ & 3190 & .0035 & .026 & $.0012-.0022$ & \\
\hline $\begin{array}{c}\text { AC Non-SOM/SOM } \\
\text { bin } 2 \text {, high arousal }\end{array}$ & 4401 & .0017 & .018 & $.0012-.0022$ & 0.00 \\
\hline $\begin{array}{c}\text { AC Non-SOM/SOM } \\
\text { bin } 2, \text { low arousal }\end{array}$ & 4401 & .0032 & .024 & $.0025-.0040$ & \\
\hline $\begin{array}{c}\text { AC Non-SOM/SOM } \\
\text { bin } 3 \text {, high arousal }\end{array}$ & 4113 & .0020 & .019 & $.0014-.0026$ & 0.00 \\
\hline
\end{tabular}




\begin{tabular}{|c|c|c|c|c|c|}
\hline $\begin{array}{c}\text { AC Non-SOM/SOM } \\
\text { bin } 3 \text {, low arousal }\end{array}$ & 4113 & .0029 & .021 & $.0023-.0036$ & .80 \\
\hline $\begin{array}{l}\text { AC Non-SOM/SOM } \\
\text { bin } 4 \text {, high arousal }\end{array}$ & 4819 & .0029 & .020 & $.0023-.0035$ & .23 \\
\hline $\begin{array}{c}\mathrm{AC} \text { Non-SOM/SOM } \\
\text { bin 4, low arousal }\end{array}$ & 4819 & .0030 & .021 & $.0024-.0036$ & \\
\hline $\begin{array}{l}\text { AC SOM/SOM bin 1, } \\
\text { high arousal }\end{array}$ & 208 & .00046 & .012 & $-.0013-.0020$ & .29 \\
\hline $\begin{array}{c}\text { AC SOM/SOM bin 1, } \\
\text { low arousal }\end{array}$ & 208 & .0025 & .022 & $-.0012-.0058$ & \\
\hline $\begin{array}{c}\text { AC SOM/SOM bin 2, } \\
\text { high arousal }\end{array}$ & 298 & .0026 & .014 & $.0010-.0042$ & .0026 \\
\hline $\begin{array}{c}\text { AC SOM/SOM bin } 2 \\
\text { low arousal }\end{array}$ & 298 & .0012 & .018 & -.00089-.0033 & \\
\hline $\begin{array}{c}\text { AC SOM/SOM bin 3, } \\
\text { high arousal }\end{array}$ & 235 & .0017 & .015 & $-.00026-.0035$ & .0002 \\
\hline $\begin{array}{c}\text { AC SOM/SOM bin 3, } \\
\text { low arousal }\end{array}$ & 235 & .0061 & .020 & $.0037-.0088$ & \\
\hline $\begin{array}{l}\text { AC SOM/SOM bin } 4, \\
\text { high arousal }\end{array}$ & 372 & .0074 & .024 & $.0051-.010$ & 0.00 \\
\hline $\begin{array}{c}\text { AC SOM/SOM bin } 4, \\
\text { low arousal }\end{array}$ & 372 & .017 & .046 & $.013-.022$ & \\
\hline $\begin{array}{l}\text { PPC Non-SOM/Non- } \\
\text { SOM bin 1, high } \\
\text { arousal }\end{array}$ & 24940 & .00088 & .015 & $.00071-.0011$ & 0.00 \\
\hline $\begin{array}{l}\text { PPC Non-SOM/Non- } \\
\text { SOM bin 1, low } \\
\text { arousal }\end{array}$ & 24940 & -.00073 & .013 & $-.00089--.00056$ & \\
\hline $\begin{array}{l}\text { PPC Non-SOM/Non- } \\
\text { SOM bin 2, high } \\
\text { arousal }\end{array}$ & 50964 & .0013 & .016 & $.0012-.0015$ & 0.00 \\
\hline $\begin{array}{l}\text { PPC Non-SOM/Non- } \\
\text { SOM bin 2, low } \\
\text { arousal }\end{array}$ & 50964 & .000083 & .014 & $-.000043-.00019$ & \\
\hline $\begin{array}{l}\text { PPC Non-SOM/Non- } \\
\text { SOM bin 3, high } \\
\text { arousal }\end{array}$ & 54231 & .0022 & .017 & $.0021-.0024$ & 0.00 \\
\hline
\end{tabular}




\begin{tabular}{|c|c|c|c|c|c|}
\hline $\begin{array}{l}\text { PPC Non-SOM/Non- } \\
\text { SOM bin 3, low } \\
\text { arousal }\end{array}$ & 54231 & .0018 & .015 & $.0017-.0019$ & \\
\hline $\begin{array}{l}\text { PPC Non-SOM/Non- } \\
\text { SOM bin 4, high } \\
\text { arousal }\end{array}$ & 46853 & .0051 & .020 & $.0049-.0053$ & 0.00 \\
\hline $\begin{array}{l}\text { PPC Non-SOM/Non- } \\
\text { SOM bin 4, low } \\
\text { arousal }\end{array}$ & 46853 & .0057 & .020 & $.0056-.0059$ & \\
\hline $\begin{array}{c}\text { PPC Non-SOM/SOM } \\
\text { bin } 1 \text {, high arousal }\end{array}$ & 4636 & .0027 & .015 & $.0022-.0031$ & .15 \\
\hline $\begin{array}{c}\text { PPC Non-SOM/SOM } \\
\text { bin } 1 \text {, low arousal }\end{array}$ & 4636 & .0031 & .017 & $.0027-.0036$ & \\
\hline $\begin{array}{c}\text { PPC Non-SOM/SOM } \\
\text { bin } 2 \text {, high arousal }\end{array}$ & 9860 & .0029 & .016 & $.0026-.0032$ & .058 \\
\hline $\begin{array}{c}\text { PPC Non-SOM/SOM } \\
\text { bin } 2 \text {, low arousal }\end{array}$ & 9860 & .0033 & .017 & $.0030-.0036$ & \\
\hline $\begin{array}{c}\text { PPC Non-SOM/SOM } \\
\text { bin } 3 \text {, high arousal }\end{array}$ & 11477 & .0034 & .017 & $.0031-.0037$ & .50 \\
\hline $\begin{array}{c}\mathrm{PPC} \text { Non-SOM/SOM } \\
\text { bin } 3 \text {, low arousal }\end{array}$ & 11477 & .0033 & .017 & $.0030-.0036$ & \\
\hline $\begin{array}{c}\text { PPC Non-SOM/SOM } \\
\text { bin } 4 \text {, high arousal }\end{array}$ & 11049 & .0038 & .018 & $.0035-.0041$ & .0061 \\
\hline $\begin{array}{c}\mathrm{PPC} \text { Non-SOM/SOM } \\
\text { bin } 4 \text {, low arousal }\end{array}$ & 11049 & .0032 & .016 & $.0029-.0035$ & \\
\hline $\begin{array}{c}\mathrm{PPC} \text { SOM/SOM bin } \\
1, \text { high arousal }\end{array}$ & 241 & -.00065 & .012 & $-.0022-.00080$ & .78 \\
\hline $\begin{array}{c}\text { PPC SOM/SOM bin } \\
1, \text { low arousal }\end{array}$ & 241 & -.00034 & .015 & $-.0025-.0012$ & \\
\hline $\begin{array}{l}\text { PPC SOM/SOM bin } \\
2, \text { high arousal }\end{array}$ & 539 & .0022 & .013 & $.0011-.0033$ & .53 \\
\hline $\begin{array}{l}\mathrm{PPC} \text { SOM/SOM bin } \\
2, \text { low arousal }\end{array}$ & 539 & .0018 & .013 & $.00068-.0027$ & \\
\hline $\begin{array}{c}\mathrm{PPC} \text { SOM/SOM bin } \\
3 \text {, high arousal }\end{array}$ & 667 & .0023 & .015 & $.0012-.0035$ & .11 \\
\hline $\begin{array}{c}\text { PPC SOM/SOM bin } \\
3 \text {, low arousal }\end{array}$ & 667 & .0035 & .015 & $.0025-.0047$ & \\
\hline
\end{tabular}


bioRxiv preprint doi: https://doi.org/10.1101/2022.01.18.476770; this version posted January 20, 2022. The copyright holder for this preprint (which was not certified by peer review) is the author/funder, who has granted bioRxiv a license to display the preprint in perpetuity. It is made available under aCC-BY-NC-ND 4.0 International license.

$\begin{array}{cccccc}\text { PPC SOM/SOM bin } & 788 & .0080 & .018 & .0068-.0094 & 0.00 \\ \text { 4, high arousal } & & & & & \\ \text { PPC SOM/SOM bin } & 788 & .012 & .023 & .011-.014 \\ \quad \begin{array}{l}\text { 4, low arousal } \\ \text { P low }\end{array} & & & & \end{array}$


Table S12: Pairwise Noise Correlations Binned By Distance (Sound Location Trials)

\begin{tabular}{|c|c|c|c|c|c|}
\hline Group & $\mathbf{N}$ & Mean & $\begin{array}{l}\text { Standard } \\
\text { Deviation }\end{array}$ & $\begin{array}{c}\text { 95\% Confidence } \\
\text { Interval of the } \\
\text { Mean }\end{array}$ & $\begin{array}{c}\text { p value (unpaired } \\
\text { permutation test, } \\
\qquad \alpha=.0125)\end{array}$ \\
\hline $\begin{array}{l}\text { AC Non-SOM/Non- } \\
\text { SOM bin } 1\end{array}$ & 27224 & .042 & .070 & $.041-.043$ & .39 \\
\hline $\begin{array}{l}\text { PPC Non-SOM/Non- } \\
\text { SOM bin } 1\end{array}$ & 92989 & .041 & .076 & $.041-.042$ & \\
\hline $\begin{array}{l}\text { AC Non-SOM/Non- } \\
\text { SOM bin } 2\end{array}$ & 50252 & .027 & .053 & $.026-.027$ & $<<.00010$ \\
\hline $\begin{array}{l}\text { PPC Non-SOM/Non- } \\
\text { SOM bin } 2\end{array}$ & 192997 & .034 & .069 & $.034-.034$ & \\
\hline $\begin{array}{l}\text { AC Non-SOM/Non- } \\
\text { SOM bin } 3\end{array}$ & 46293 & .020 & .046 & $.019-.020$ & $<<.00010$ \\
\hline $\begin{array}{l}\text { PPC Non-SOM/Non- } \\
\text { SOM bin } 3\end{array}$ & 181712 & .031 & .066 & $.031-.032$ & \\
\hline $\begin{array}{l}\text { AC Non-SOM/Non- } \\
\text { SOM bin } 4\end{array}$ & 36472 & .19 & .044 & $.019-.020$ & $<<.00010$ \\
\hline $\begin{array}{l}\text { PPC Non-SOM/Non- } \\
\text { SOM bin } 4\end{array}$ & 152621 & .029 & .064 & $.029-.030$ & \\
\hline $\begin{array}{c}\text { AC Non-SOM/SOM } \\
\text { bin } 1\end{array}$ & 6755 & .044 & .071 & $.042-.046$ & .0092 \\
\hline $\begin{array}{c}\text { PPC Non- } \\
\text { SOM/SOM bin } 1\end{array}$ & 19046 & .047 & .079 & $.046-.048$ & \\
\hline $\begin{array}{c}\text { AC Non-SOM/SOM } \\
\text { bin } 2\end{array}$ & 13384 & .027 & .055 & $.027-.028$ & $<<.00010$ \\
\hline $\begin{array}{c}\text { PPC Non- } \\
\text { SOM/SOM bin } 2\end{array}$ & 39303 & .040 & .073 & $.040-.041$ & \\
\hline $\begin{array}{c}\text { AC Non-SOM/SOM } \\
\text { bin } 3\end{array}$ & 12334 & .020 & .049 & $.019-.021$ & $<<.00010$ \\
\hline $\begin{array}{c}\text { PPC Non- } \\
\text { SOM/SOM bin } 3\end{array}$ & 37849 & .038 & .071 & $.038-.039$ & \\
\hline $\begin{array}{c}\text { AC Non-SOM/SOM } \\
\text { bin } 4\end{array}$ & 9000 & .018 & .046 & $.017-.019$ & $<<.00010$ \\
\hline $\begin{array}{c}\text { PPC Non- } \\
\text { SOM/SOM bin } 4\end{array}$ & 33121 & .039 & .072 & $.038-.040$ & \\
\hline AC SOM/SOM bin 1 & 513 & .058 & .086 & $.051-.066$ & .00050 \\
\hline
\end{tabular}




\begin{tabular}{|c|c|c|c|c|c|}
\hline $\begin{array}{c}\mathrm{PPC} \text { SOM/SOM bin } \\
1\end{array}$ & 984 & .078 & .11 & $.071-.085$ & \\
\hline AC SOM/SOM bin 2 & 891 & .036 & .066 & $.032-.041$ & $<.00010$ \\
\hline $\begin{array}{c}\mathrm{PPC} \text { SOM/SOM bin } \\
2\end{array}$ & 2412 & .074 & .11 & $.070-.078$ & \\
\hline AC SOM/SOM bin 3 & 830 & .025 & .061 & $.021-.029$ & $<.00010$ \\
\hline $\begin{array}{c}\mathrm{PPC} \text { SOM/SOM bin } \\
3\end{array}$ & 2277 & .066 & .10 & $.063-.070$ & \\
\hline $\mathrm{AC}$ SOM/SOM bin 4 & 554 & .016 & .045 & $.012-.020$ & $<.00010$ \\
\hline $\begin{array}{c}\mathrm{PPC} \text { SOM/SOM bin } \\
4\end{array}$ & 1971 & .071 & .10 & $.067-.076$ & \\
\hline
\end{tabular}


Table S13: Noise Correlation Spatial Decay Constants (Sound Location Trials)

\begin{tabular}{|c|c|c|c|c|c|}
\hline Group & $\mathbf{N}$ & Mean & $\begin{array}{l}\text { Standard } \\
\text { Deviation }\end{array}$ & $\begin{array}{l}\text { 95\% Confidence } \\
\text { Interval of the } \\
\text { Mean }\end{array}$ & p value \\
\hline $\mathrm{AC}$ all $\Lambda^{1}$ & 71 & .0041 & .0037 & $.0033-.0050$ & \\
\hline PPC all $\kappa^{2}$ & 59 & .00079 & .0014 & $.0005-.0012$ & \\
\hline Unpaired permutation ${ }^{1,2}$ & & & & & $<.00010$ \\
\hline AC Non-SOM/Non-SOM $\kappa^{3}$ & 24 & .0035 & .0018 & $.0028-.0042$ & \\
\hline AC SOM/Non-SOM $\kappa^{4}$ & 24 & .004 & .0023 & $.0031-.0049$ & \\
\hline $\mathrm{AC} \mathrm{SOM} / \mathrm{SOM} \Lambda^{5}$ & 23 & .0049 & .0057 & $.0028-.0073$ & \\
\hline Kruskal Wallis ${ }^{3-5}$ & & & & & .61 \\
\hline PPC Non-SOM/Non-SOM $\kappa^{6}$ & 20 & .00092 & .00070 & $.00070-.0013$ & \\
\hline PPC SOM/Non-SOM $\kappa^{7}$ & 20 & .00047 & .001 & $-.00011-.00055$ & \\
\hline $\mathrm{PPC} \mathrm{SOM} / \mathrm{SOM} \kappa^{8}$ & 19 & .00098 & .0021 & $.00030-.0022$ & \\
\hline Kruskal Wallis ${ }^{6-8}$ & & & & & .47 \\
\hline Kruskal Wallis ${ }^{3-8}$ & & & & & $1.96 \mathrm{E}-09$ \\
\hline
\end{tabular}


Table S14: Pairwise Noise Correlations (Running Bout Trials)

\begin{tabular}{|c|c|c|c|c|c|}
\hline Group & $\mathbf{N}$ & Mean & $\begin{array}{l}\text { Standard } \\
\text { Deviation }\end{array}$ & $\begin{array}{c}95 \% \\
\text { Confidence } \\
\text { Interval of the } \\
\text { Mean }\end{array}$ & p value \\
\hline $\mathrm{AC}$ all neurons ${ }^{1}$ & 204502 & .016 & .089 & $.016-.017$ & \\
\hline PPC all neurons ${ }^{2}$ & 757282 & .025 & .11 & $.025-.025$ & \\
\hline Unpaired permutation ${ }^{1,2}$ & & & & & $<.00010$ \\
\hline $\begin{array}{c}\text { AC Non-SOM/Non- } \\
\text { SOM }^{3}\end{array}$ & 160241 & .017 & .090 & $.016-.017$ & \\
\hline AC Non-SOM/SOM ${ }^{4}$ & 41473 & .015 & .084 & $.014-.015$ & \\
\hline $\mathrm{AC} \mathrm{SOM} / \mathrm{SOM}^{5}$ & 2788 & .017 & .084 & $.014-.020$ & \\
\hline Kruskal Wallis ${ }^{3-5}$ & & & & & .10 \\
\hline $\begin{array}{c}\text { PPC Non-SOM/Non- } \\
\text { SOM }^{6}\end{array}$ & 620319 & .025 & .11 & $.024-.025$ & \\
\hline PPC Non-SOM/SOM ${ }^{7}$ & 129319 & .025 & .10 & $.024-.025$ & \\
\hline PPC SOM/SOM ${ }^{8}$ & 7644 & .039 & .11 & $.037-.042$ & \\
\hline Kruskal Wallis ${ }^{6-8}$ & & & & & 2.39E-41 \\
\hline Unpaired permutation ${ }^{6,7}$ & & & & & .82 \\
\hline Unpaired permutation 7,8 & & & & & $<.00010$ \\
\hline Unpaired permutation 6,8 & & & & & $<.00010$ \\
\hline Kruskal Wallis ${ }^{3-8}$ & & & & & $6.3 \mathrm{E}-225$ \\
\hline
\end{tabular}


Table S15: Pairwise Noise Correlations Binned By Distance (Running Bout Trials)

\begin{tabular}{|c|c|c|c|c|c|}
\hline Group & $\mathbf{N}$ & Mean & $\begin{array}{l}\text { Standard } \\
\text { Deviation }\end{array}$ & $\begin{array}{l}\text { 95\% Confidence } \\
\text { Interval of the } \\
\text { Mean }\end{array}$ & $\begin{array}{c}\text { p value } \\
\text { (unpaired } \\
\text { permutation } \\
\text { test, } \alpha=.0125 \text { ) }\end{array}$ \\
\hline $\begin{array}{l}\text { AC Non-SOM/Non- } \\
\text { SOM bin } 1\end{array}$ & 27224 & .028 & .10 & $.027-.029$ & $<.00010$ \\
\hline $\begin{array}{c}\text { PPC Non- } \\
\text { SOM/Non-SOM bin } \\
1\end{array}$ & 92989 & .032 & .12 & $.031-.033$ & \\
\hline $\begin{array}{l}\text { AC Non-SOM/Non- } \\
\text { SOM bin } 2\end{array}$ & 50252 & .018 & .091 & $.017-.019$ & $<.00010$ \\
\hline $\begin{array}{c}\text { PPC Non- } \\
\text { SOM/Non-SOM bin } \\
2\end{array}$ & 192997 & .026 & .11 & $.025-.026$ & \\
\hline $\begin{array}{l}\text { AC Non-SOM/Non- } \\
\text { SOM bin } 3\end{array}$ & 46293 & .014 & .087 & $.013-.015$ & $<.00010$ \\
\hline $\begin{array}{c}\text { PPC Non- } \\
\text { SOM/Non-SOM bin } \\
3\end{array}$ & 181712 & .023 & .11 & $.022-.023$ & \\
\hline $\begin{array}{c}\text { AC Non-SOM/Non- } \\
\text { SOM bin } 4\end{array}$ & 36472 & .010 & .084 & $.0095-.011$ & $<.00010$ \\
\hline $\begin{array}{c}\text { PPC Non- } \\
\text { SOM/Non-SOM bin } \\
4\end{array}$ & 152621 & .021 & .11 & $.020-.022$ & \\
\hline $\begin{array}{l}\text { AC Non-SOM/SOM } \\
\text { bin } 1\end{array}$ & 6755 & .027 & .093 & $.025-.030$ & $<.00010$ \\
\hline $\begin{array}{c}\text { PPC Non- } \\
\text { SOM/SOM bin } 1\end{array}$ & 19046 & .031 & .11 & $.029-.032$ & \\
\hline $\begin{array}{c}\text { AC Non-SOM/SOM } \\
\text { bin } 2\end{array}$ & 13384 & .016 & .085 & $.014-.017$ & $<.00010$ \\
\hline $\begin{array}{c}\text { PPC Non- } \\
\text { SOM/SOM bin } 2\end{array}$ & 39303 & .026 & .10 & $.025-.027$ & \\
\hline $\begin{array}{c}\text { AC Non-SOM/SOM } \\
\text { bin } 3\end{array}$ & 12334 & .010 & .080 & $.0091-.012$ & $<.00010$ \\
\hline $\begin{array}{c}\text { PPC Non- } \\
\text { SOM/SOM bin } 3\end{array}$ & 37849 & .023 & .10 & $.022-.024$ & \\
\hline
\end{tabular}




$\begin{array}{cccccc}\begin{array}{c}\text { AC Non-SOM/SOM } \\ \text { bin } 4\end{array} & 9000 & .0094 & .079 & .0076-.011 & <.00010 \\ \begin{array}{c}\text { PPC Non- } \\ \text { SOM/SOM bin } 4\end{array} & 33121 & .022 & .10 & .021-.023 & \\ \begin{array}{c}\text { AC SOM/SOM bin } \\ 1\end{array} & 513 & .032 & .094 & .024-.040 & .017 \\ \begin{array}{c}\text { PPC SOM/SOM bin } \\ 1\end{array} & 984 & .047 & .12 & .040-.054 & <.00010 \\ \begin{array}{c}\text { AC SOM/SOM bin } \\ 2\end{array} & 891 & .025 & .092 & .020-.032 & \\ \begin{array}{c}\text { PPC SOM/SOM bin } \\ 2\end{array} & 2412 & .041 & .11 & .037-.046 & \\ \begin{array}{c}\text { AC SOM/SOM bin } \\ 3\end{array} & 830 & .0094 & .075 & .0041-.014 & \\ \begin{array}{c}\text { PPC SOM/SOM bin } \\ 3\end{array} & 2277 & .035 & .10 & .031-.039 & \\ \begin{array}{c}\text { AC SOM/SOM bin } \\ 4\end{array} & 554 & .0027 & .066 & -.0026-.0090 & \\ \text { PPC SOM/SOM bin } & 1971 & .038 & .10 & .034-.043 & \end{array}$


Table S16: Noise Correlation Spatial Decay Constants (Running Bout Trials)

$\begin{array}{lcccc}\text { Group } & \text { N } & \text { Mean } & \begin{array}{c}\text { Standard } \\ \text { Deviation }\end{array} & \begin{array}{c}\mathbf{9 5 \%} \\ \text { Confidence } \\ \text { Interval of the } \\ \text { Mean }\end{array} \\ \text { AC all } \Lambda^{1} & 71 & .0037 & .0045 & .0027-.0048 \\ \text { PPC all } \kappa^{2} & 59 & .0011 & .0017 & .0007-.0015\end{array}$

Unpaired permutation ${ }^{1,2}$

$\begin{array}{ccccc}\mathrm{AC} \mathrm{Non-SOM} / \mathrm{Non}-\mathrm{SOM} \kappa^{3} & 24 & .0030 & .0026 & .0020-.0042 \\ \mathrm{AC} \mathrm{SOM} / \mathrm{Non}-\mathrm{SOM} \kappa^{4} & 24 & .0038 & .0037 & .0023-.0054 \\ \mathrm{AC} \mathrm{SOM} / \mathrm{SOM} \kappa^{5} & 23 & .0047 & .0072 & .0013-.0082\end{array}$

Kruskal Wallis ${ }^{3-5}$

$\begin{array}{ccccc}\text { PPC Non-SOM/Non-SOM } \kappa^{6} & 20 & <.00010 & .0013 & .00038-.0015 \\ \text { PPC SOM/Non-SOM } \Lambda^{7} & 20 & <.00010 & .0014 & .00048-.0017 \\ \text { PPC SOM/SOM } \Lambda^{8} & 19 & .0012 & .0024 & .00028-.0023\end{array}$

Kruskal Wallis ${ }^{6-8}$

Kruskal Wallis ${ }^{3-8}$ 
Table S17: Coupling

Group

AC Non-SOM/Non$\mathrm{SOM}^{1}$

AC Non-SOM/SOM ${ }^{2}$ AC SOM/SOM ${ }^{3}$
PPC Non-SOM/Non- $\mathrm{SOM}^{4}$
PPC Non-SOM/SOM ${ }^{5}$ PPC SOM/SOM ${ }^{6}$

Kruskal Wallis ${ }^{1-6}$

Unpaired permutation ${ }^{1,2}$

Unpaired permutation ${ }^{1,3}$

Unpaired permutation ${ }^{1,4}$

Unpaired permutation ${ }^{1,5}$

Unpaired permutation ${ }^{1,6}$

Unpaired permutation ${ }^{2,3}$

Unpaired permutation ${ }^{2,4}$

Unpaired permutation ${ }^{2,5}$

Unpaired permutation ${ }^{2,6}$

Unpaired permutation ${ }^{3,4}$

Unpaired permutation ${ }^{3,5}$

Unpaired permutation ${ }^{3,6}$

Unpaired permutation ${ }^{4,5}$

Unpaired permutation ${ }^{4,6}$

Unpaired permutation ${ }^{5,6}$
$\mathbf{N}$

Mean

Standard
Deviation

2428

320

327

4048

485

484

0.0022

0.0074

0.0019

0.0037

0.0057

0.0112

$0.0015-0.0027$

0.0027-0.0052

\section{Interval of the}

Mean

0.0015-0.0020

0.0057

0.0031

0.0112

0.0028-0.0035

0.0101

0.0033-0.0051

0.0193

0.0058-0.0092 p value

$<0.0001$

$<0.0001$

$<0.0001$

$<0.0001$

$<0.0001$

$<0.0001$

$<0.0001$

$<0.0001$

$<0.0001$

$<0.0001$

$<0.0001$

$<0.0001$

$<0.0001$

$<0.0001$

$<0.0001$

$<0.0001$ 